Portland State University

PDXScholar

$1-1-1980$

\title{
Studies of homogeneous nucleation and transient heat transfer in cryogenic liquids
}

Dipen N. Sinha

Portland State University

Follow this and additional works at: https://pdxscholar.library.pdx.edu/open_access_etds Let us know how access to this document benefits you.

\section{Recommended Citation}

Sinha, Dipen N., "Studies of homogeneous nucleation and transient heat transfer in cryogenic liquids" (1980). Dissertations and Theses. Paper 596.

https://doi.org/10.15760/etd.596

This Dissertation is brought to you for free and open access. It has been accepted for inclusion in Dissertations and Theses by an authorized administrator of PDXScholar. Please contact us if we can make this document more accessible: pdxscholar@pdx.edu. 
STUDIES OF HOMOGENEOUS NUCLEATION AND

TRANSIENT HEAT TRANSFER IN CRYOGENIC LIQUIDS

by

DIPEN N. SINHA

A dissertation submitted in partial fulfillment of the requirements for the degree of

DOCTOR OF PHILOSOPHY

in

ENVIRONMENTAL SCIENCES AND RESOURCES - PHYSICS

Portland State University

1980 
TO THE OFFICE OF GRADUATE STUDIES AND RESEARCH:

The members of the Committee approve the dissertation of

Dipen N. Sinha presented June 3, 1980.
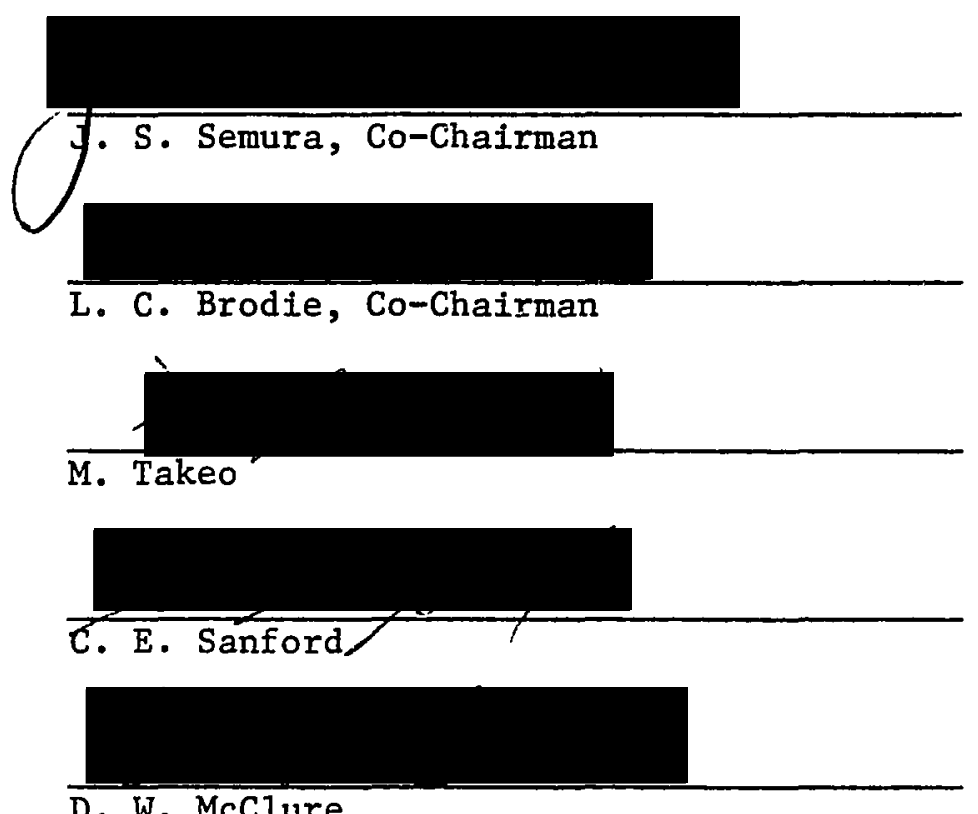

D. W. McClure

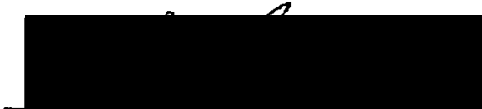

S. S. HIllman

APPROVED :

M. Gurevitch, Head, Department of Physics

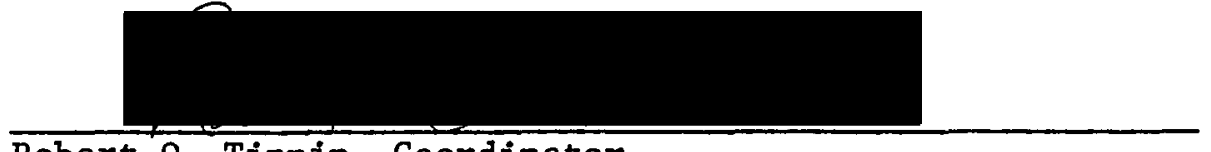

Robert 0. Tinnin, Coordinator,

Environmental Sciences and Resources Ph.D. Program

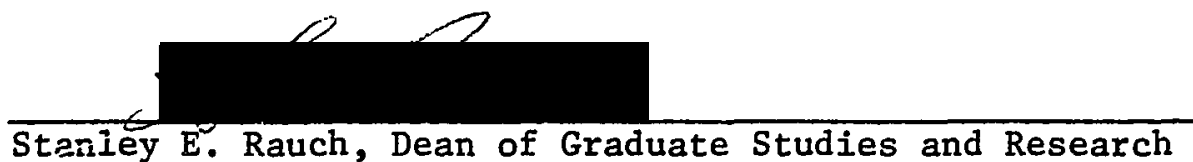


AN ABSTRACT OF THE DISSERTATION OF Dipen N. Sinha for the

Doctor of Philosophy in Environmental Sciences and Resources - Physics presented June 3, 1980 .

Title: Studies of Homogeneous Nucleation and Transient

Heat Transfer in Cryogenic Liquids

APPROVED BY MEMBERS OF THE DISSERTATION COMMITTEE:
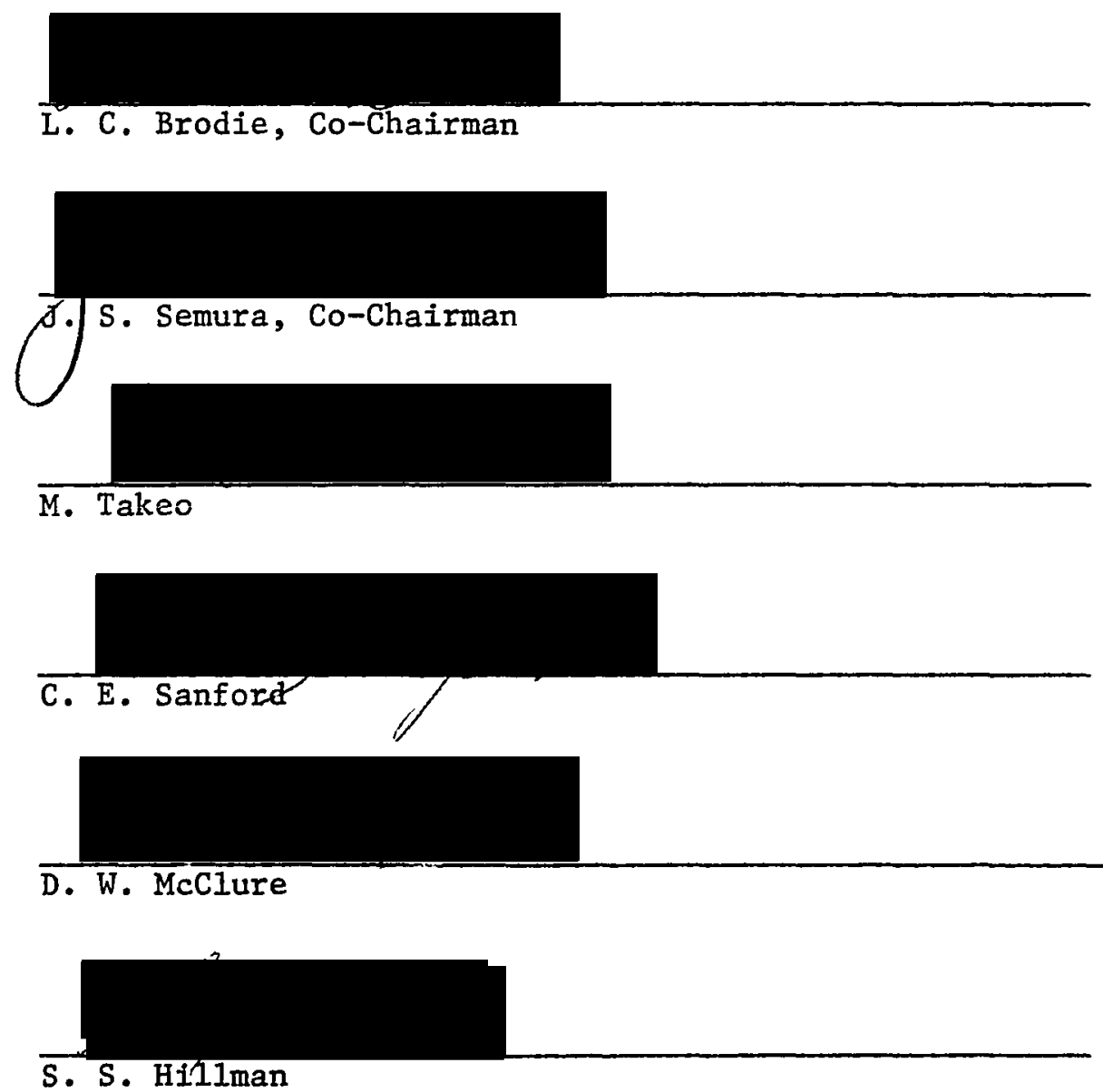
ABSTRACT

We report the results of a series of experimental studies in two interrelated areas: homogeneous nucleation and transient heat transfer in cryogenic liquids. We report the first determination of the homogeneous nucleation temperature of liquid helium I and liquid nitrogen employing a transient superheating technique. This technique in Iiquid helium involved the development of a new transient magnetoresistive thermometer which relies on the strong temperature dependence of magnetoresistance in bismuth as a thermometric property. Our measurements of the homogeneous nucleation temperature for both liquid helium $I$ and nitrogen are in good agreement with the predictions from the Becker-Doring-Volmer-Zel'dovich-Frenkel nucleation theory. On the basis of such agreement between theory and experiment in both liquid helium and liquid nitrogen we have developed a corresponding state analysis applicable to the case of homogeneous nucleation in liquid noble gases, both quantum and classical. Predictions of the homogeneous nucleation temperature and surface tension of several hydrogen isotopes are made from such a quantum mechanical law of corresponding states. We also comment on the limiting superheat temperature of liquid helium I. It is suggested that a comparison between the limiting superheat temperature and the peak nucleate boiling temperature is meaningless and that the reported agreement between the two is largely fortuitous. In the studies of transient heat transfer in helium we find that the heat transfer from a solid into liquid helium is markedly enkanced by the application of a visible pulse of light. In liquid nitrogen we find that a transition to stable film boiling can be observed for power values as low as 40 per cent of the steady-state peak heat flux. 


\section{ACKNOWLEDGEMENTS}

This dissertation is dedicated to my parents for their inspiration and support, and to my wife Barbara who has been an incomparable source of encouragement. I owe a great deal to Dr. Laird Brodie who taught me much about research. His enthusiasm and his openness to discussing with me even the most far-fetched ideas helped to make these six years extremeIy enjoyable. My deep gratitude is to Dr. Jack Semura who has given me the benefit of his insight and incisive opinion over the years. Through his helpful criticisms and friendly suggestions he helped me overcome many of my weaknesses.

It is indeed a pleasure to acknowledge my good friend Dr. Shripad Tuljapurkar who has helped me in myriad ways. Many thanks are due Dr. Fred Young for numerous helpful suggestions in the initial phases of this work. I am grateful to many people with whom I have discussed various aspects of my research, most especially to Dr. Makoto Takeo, Dr. Pavel Smejtek, and Dr. Gertrude Rempfer. I thank Dr. Mark Gurevitch for his continual support and Mrs. Dawn Dressler for her kindness.

I will always be grateful to Mr. Lee Thannum, Mr. Brian McLaughlin, Mr. Jack Janacek, Mr. Rudi Zupan, and Mr. Garo Arakelian for their invaluable technical assistance without which much of this work could not have been done. Finally I thank Dr. J. J. Kohut. Mr. R. Lockerby, and Mrs. I. Kajiwara for their friendliness and cooperation and Mrs. E. Crowell for her help in obtaining numerous journal articles through inter-library loan. 
TABLE OF CONTENTS

$\underline{\text { PAGE }}$

ACKNOWLEDGMENTS ................. . i ii

LIST OF TABLES . . . . . . . . . . . . . vii LIST OF FIGURES . . . . . . . . . . . . . . . viii

CHAPTER

I INTRODUCTION . . . . . . . . . . . 1

Transient thermometry

Homogeneous nucleation

Effect of light on heat transfer

Transient heat transfer

II TRANSIENT MAGNETORESISTIVE THERMOMETRY IN

LIQUID HELIUM $\cdot$. . . . . . . . • 11

Introduction . . . . . . . . . . 11

Experimental Details........... 18

Thermometer construction

Circuit details

Calibration

Factors Affecting the Temperature

Sensitivity of the Crystal ........ 26

Quality

Magnetic field

Crystal orientation

Features of the Bismuth Thermometer . . . 30

Accuracy

Response time

Sensitivity

Range

Specific applications and advantages 
III CORRESPONDING STATES BEHAVIOR OF LIQUID-TO-

VAPOR NUCIEATION IN CLASSICAL AND QUANTUM

LIQUIDS

Introduction ............ 4 40

Theoretical Background .......... 43

Experimental Techniques ........ 49

Exploding droplet method

Pulse heating method

Transient superheating method

Observations . . . . . . . . . . .

Discussion of Experimental Interpretations .

Results ............ 69

Corresponding States Analysis . . . . . 73

IV HOMOGENEOUS NUCLEATION IN NITROGEN . . . . 85

Introduction . . . . . . . . 85

Apparatus and Experimental Procedure . . . 89

Transient Superheating Technique . . . . . 93

Results and Discussion . . . . . . . 95

Conclusion . . . . . . . . . 105

$\mathrm{V}$ COMMENTS ON THE LIMITING SUPERHEAT TEMPERATURE

IN LIQUID HELIUM I . . . . . . . . . . . 106

VI IIGHT INDUCED ENHANCEMENT OF TRANSIENT HEAT

TRANSFER IN HELIUM . . . . . . . . . . 121

Introduction . . . . . . . . . . 121

Experimental Details . . . . . . . 123

Description of Light-Pulse Induced

Enhancement .............. 124

Heat transfer enhancement 
Discussion ........... . 132

Photoelectron bubble hypothesis

Phonon hypothesis

Significance . . . . . . . . . . . 148

VII PREMATURE TRANSITION TO STABLE FIIM BOILING

INITIATED BY POWER TRANSIENTS IN NITROGEN • 150

Introduction . . . . . . . . . . 150

Experimental . . . . . . . . . . 153

Measurement of superheat temperature

Measurement of applied power and heat flux

Results and Discussion ........ 161

REFERENCES • • • • • • • . • • • • • • • • • • • 176

APPENDIX A . . . . . . . . . . . . . . . . . . . . 184

APPENDIX B . . . . . . . . . . . . . . . . . . . 191

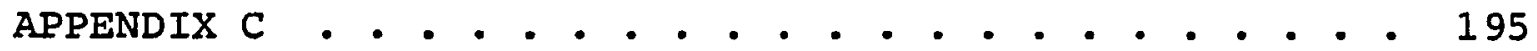




\section{IIST OF TABLES}

TABLE

PAGE

I Homogeneous Nucleation Temperature of Liquid Helium . . . . . . . . . . . 65

II The Molecular Parameters for 6-12 LennardJones Fluids.... . . . . . . . . 76

III The Critical Power, steady-State Heat Flux, and the Minimum Value of the Peak Transient Heat Flux 


\section{LIST OF FIGURES}

FIGURE

$\underline{\text { PAGE }}$

1. Transverse magnetoresistance of a single crystal of bismuth . . . . . . . . . 17

2. Experimental set-up . . . . . . . . . 20

3. Schematic circuit diagram . . . . . . . . 22

4. Calibration curve for bismuth crystals . . . 27

5. Superheat temperature $\Delta \mathrm{T}$ vs. time . . . . . 31

6. Superheat temperature $\Delta \mathrm{T}$ vs. time . . . . . 55

7. Maximum superheat temperature as a function of applied power . . . . . . . . . . 57

8. Temperature dependence of $\mathrm{J}$ and $\mathrm{w}_{\mathrm{cr}} \cdot \bullet^{-} \cdot{ }^{-} \quad 64$

9. Scaled limits of superheat, $\mathrm{T}_{\mathrm{h}} / \mathrm{T}_{\mathrm{C}}$ vs. scaled pressure, $\mathrm{P} / \mathrm{P}_{\mathrm{C}} \cdot$. . . . . . . . . . 77

10. Reduced homogeneous nucleation temperature $\mathrm{T}_{\mathrm{h}}^{*}$ vs. reduced pressure $\mathrm{P}^{*}$. . . . . . . . 79

11. A plot of equations (3.15) and (3.16) . . . 81

12. A plot of equation (3.17) . . . . . . . . 83

13. Schematic diagram of the cryostat assembly . . 90

14. Superheat temperature $\Delta \mathrm{T}$ vs. time . . . . . 96

15. Superheat temperature and laser beam
intensity vs. time . . . . . . . 100

16. Scaled homogeneous nucleation temperature
$\mathrm{T}_{\mathrm{h}} / \mathrm{T}_{\mathrm{C}}$ vs. scaled pressure $\mathrm{P} / \mathrm{P}_{\mathrm{C}} \cdot \bullet^{-} \cdot \bullet^{-} \cdot{ }^{-}$

17. Temperature dependence of the nucleation
rate . . . . . . . . . . . . 104 
18. Spinodal for Argon, Hydrogen, and Helium-4 as determined from Furth's hole theory . . 112

19. Superheat temperature at critical heat flux as a function of bath temperature . . . . 116

20. Superheat temperature $\Delta \mathrm{T}$ vs. time . . . . . 125

21. Effect of pulsed light on the $\Delta \mathrm{T}$ vs. time curve . . . . . . . . . . . . 125

22. Effect of pulsed light on the transient superheat temperature characteristics . . . 127

23. Composite record of ten $\Delta T$ vs. time curves . - 127

24. $\Delta \mathrm{T}$ vs. time curves at two different heating rates ................... 129

25. Effect of light pulse intensity on $\Delta \mathrm{T}$ vs. time curve.......... . . . . . . 129

26. Effect of steady room light on the $\Delta T$ vs. time curve.............. . . 131

27. $\Delta \mathrm{T}_{\mathrm{IT}}$ as a function of applied powers . . . 134

28. Homogeneous nucleation temperature as a function of pressure . . . . . . . . 138

29. $\Delta \mathrm{T}_{\mathrm{IT}}$ as a function of bath temperature . . - 140

30. Energy level diagram for an electron in the vicinity of the bismuth-helium interface . . 142

31. Schematic representation of the experimental set-up . . . . . . . . . . . . . 144

32. Superheat temperature $\Delta \mathrm{T}$ vs. time . . . . . 144

33. Schematic circuit diagram . . . . . . . 155

34. Superheat temperature $\Delta \mathrm{T}$ vs. time . . . . . 162

35. Heat flux $q$ and superheat temperature
$\Delta \mathrm{T}$ vs. time 167

36. Heat flux q vs. superheat temperature $\Delta T$. . 169 
37. Critical power $p_{c r}$ vs. heater power . • • • 174

38. Surface tension of deuterium as a function of temperature... . . . . . . . . 188

39. A plot of $\mu$ and $\alpha$ as a function of the de Boer parameter . . . . . . . . . 189 
CHAPTER I

\section{INTRODUCTION}

This dissertation describes the results of a series of experimental studies in two interrelated areas: (1) homogeneous nucleation, and (2) transient heat transfer in cryogenic liquids. These studies have been specifically directed toward liquid helium I and liquid nitrogen. In the study of homogeneous nucleation our main emphasis has been on the determination of the homogeneous nucleation temperature in superheated liquid helium I and liquid nitrogen. Such measurements are new and appear to have not been carried out before. A transient superheating method was employed as the underlying experimental basis for these investigations. This methodology in liquid helium involved the development of a new transient magnetoresistive thermometry technique which exploits the strong temperature dependence of magnetoresistance of single crystals of bismuth at liquid helium temperatures as a thermometric property. Our measurements of the homogeneous nucleation temperature are in good agreement with the predictions from the Becker-Doring-Volmer-Zel'dovich-Frenkel (BDVZF) nucleation theory. Such agreement between the BDVZF theory and the experimental results for a quantum fluid such as liquid helium led us to develop a corresponding states analysis 
applicable to the case of homogeneous nucleation for both quantum and classical fluids. Predictions of the homogeneous nucleation temperature and surface tension of several hydrogen isotopes are made on the basis of such a quantum mechanical law of corresponding states. Our investigations also suggested that the homogeneous nucleation temperature in liquid helium $I$ is not likely to be determined under steady-state heat transfer conditions.

In the studies of transient heat transfer an effect was discovered in which the transient heat transfer from a solid into liquid helium was enhanced markedly by the application of a visible light pulse of roughly $40 \mu \mathrm{s}$ in duration. In liquid nitrogen we find that a transition to stable film boiling can be observed for power values as low as about 40 per cent of the steady-state peak heat flux. We also demonstrate that such a premature transition to film boiling can be inhibited by forced convection in the liquid.

There are a number of environmental and resources considerations that are involved in the study of homogeneous nucleation and transient heat transfer in cryogenic liquids. Work on homogeneous nucleation contributes to an understanding of the homogeneous nucleation process in general. A knowledge of the homogeneous nucleation process in a quantum fluid such as liquid helium is of fundamental significance in realizing the limitations and restrictions of the BDVZF nucleation theory. This theory provides a 
common conceptual basis for all the nucleation processes that are encountered in our environment through the crystallization of supercooled liquids, condensation of supersaturated vapors, boiling of superheated liquids, and cavitation of stressed liquids.

We now focus our discussion specifically on one of the nucleation processes mentioned above, namely, boiling of superheated liquids. Investigations in the area of superheated liquids close to their homogeneous nucleation limit are no longer a scientific curiosity only but involve important environmental considerations. It is well known that if boiling is initiated in a highly superheated liquid the liquid will boil explosively. Such explosive boiling presents a safety hazard in industry and is considered to be involved in the dangerous and destructive explosive phenomenon known as contact vapor explosion observed in many metallurgical processes and in paper smelt processing (Blander and Katz 1973, Bradley and Witte 1972, Brauer et al. 1968, Flory et al. 1969, Genco and Lemmon 1970, Long 1957, Nelson and Kennedy 1956, witte et al. 1970). Explosive boizing also poses a potential hazard in the transport of liquefied natural gas (ING) and in the operation of nuclear reactors (Burgess et al. 1970, Enger and Hartman 1972, Fauske 1972, Katz 1972, Katz and Slipcevich 1971, Nakanishi and Reid 1971). The liquid natural gas vapor explosions are nonchemical flameless explosions that sometimes occur when ING is spilled on a warmer fluid. 
It is thought that vapor explosions occur when cool liquids are suddenly warmed to their homogeneous nucleation temperature (Katz and Slipcevich 1971, Enger et al. 1973) and therefore an understanding of liquid to vapor homogeneous nucleation process in cryogenic liquids is of considerable basic importance. It should be mentioned in this connection that in contrast to the extensive work carried out studying homogeneous nucleation in a vast number of organic liquids, such investigations in liquid helium and liquid nitrogen are completely lacking.

Our work in liquid helium $I$ is relevant in the design of superconducting magnets and devices. Large superconducting magnets are currently of considerable interest in view of their importance in energy storage. Magnetohydrodynamics, fusion and in the development of magnetic levitation and propulsion for high speed transportation (Bocherts 1975, Kyotami 1975, Rhodes and Mulhall 1975, Oshima 1974). The economic justifications for most large magnet systems rests upon the feasibility of large superconducting magnets. The most common type of superconducting magnet relies on pool boiling heat transfer in liquid helium for its cryostatic stabilization. However, it has been gradually recognized that the steady-state analysis of superconducting magnets must be modified by applying transient heat transfer parameters. The study of transient heat transfer in liquid helium is therefore of importance in assessing the dynamic behavior and safe limits in the 
design and operation of superconducting magnets and devices. It is worth pointing out that not only transient heat transfer but homogeneous nucleation in liquid helium is also relevant to the design of superconducting magnets. The explosive boiling associated with homogeneous nucleation in liquid helium may perhaps play a significant role in exacerbating the effects of heat pulses appearing in cooling channels of superconducting magnets. If, for example, the channel is narrow enough to be completely filled with superheated liquid helium, a very small heat pulse may then be sufficient to trigger explosive homogeneous nucleation. At the very least the channel will then be completely emptied of liquid, rendering it incapable of providing further refrigeration until it has been refilled. It is also conceivable that pressures developed during the explosive production of vapor in the superheated liquid may be sufficient to slightly push out the walls, thus triggering additional mechanical disturbances.

The importance of liquid nitrogen stems from its wide range of application in present day technology. These applications include the cryopreservation of biological materials, low noise amplifiers for satellite communication systems, cryogenic pumping of gases, and cryoresistive transmission lines. The primary mode of use of liquid nitrogen is as a refrigerant. This underlines the need for the study of heat transfer characteristics of this fluid. Although extensive work has been done on the steady-state heat 
transfer, very little work is reported on the transient heat transfer in liquid nitrogen. It is of significant interest to study the influence of power transients on the effectiveness of the heat transfer involved in the cryogenic devices refrigerated in liquid nitrogen.

In the remainder of this chapter we summarize the content of this dissertation and discuss briefly the significance of each chapter.

\section{Transient thermometry}

An initial difficulty in studying transient heat transfer in liquid helium, particularly in the interesting regime of nucleate boiling, was that no accurate and sensitive thermometers were available which could be used for fast transient temperature measurements at such temperatures. In Chapter II we describe the development of a new fast transient magnetoresistive thermometry technique particularly suitable for studying both the inception of heterogeneous nucleation in liquid helium and the homogeneous nucleation temperature of liquid helium.

\section{Homogeneous nucleation}

Homogeneous nucleation in helium has involved a controversy in that previous measurements of cavitation strength yielded results in complete disagreement with the so-called 'classical' nucleation theory. It was consequently assumed that classical homogeneous nucleation theory might be invalid in a quantum liquid such as helium and 
that a different mechanism of nucleation might be involved. We therefore planned to study homogeneous nucleation in liquid helium directly. We used the transient thermometry technique described in Chapter II to measure the homogeneous nucleation temperature of helium over a wide range of saturation bath temperatures. This is the first such measurement for a quantum fluid. Our results clearly show that the 'classical' nucleation theory is valid for helium and no other mechanism of nucleation is involved. The results and the technique are discussed in chapter III. The technique we developed to determine the homogeneous nucleation temperature also showed that it is possible to superheat a liquid up to its homogeneous nucleation limits, even in the presence of a solid surface, if the superheating is done quite fast (in the order of milliseconds). Such high superheat becomes attainable because of the relativeiy iong delay times involved for the onset of convection and inception of heterogeneous nucleation. An interesting by-product of this technique is that since in the initial stages of superheating the only mode of energy transport through the solid-liquid interface is by thermal conduction, the thermal conductivity of the fluid can be easily determined from the transient temperature response of the heater-thermometer.

In Chapter IV we present the first measurements of homogeneous nucleation temperature in liquid nitrogen over a wide range of reduced pressures $P / P_{C}$, roughly from .03 
to .91, using the transient superheating technique. Theoretical predictions of the effects of a homogeneous magnetic field on the maximum attainable superheat of a paramagnetic fluid such as liquid oxygen is given in Appendix $B$.

An interesting generalization can be made from the preceding studies. We found that the homogeneous nucleation temperature of condensed noble gases, both classical and quantum, obey a principle of corresponding states. Based on this result, we have developed a quantum mechanical law of corresponding states applicable to the case of homogeneous nucleation, and used this to predict homogeneous nucleation temperatures of several hydrogen isotopes for the first time. Such predictions are difficult to make using the nucleation theory because the required thermophysical properties for these isotopes are not readily available. These results are also presented in Chapter III. In addition, we have been able to predict surface tension data for hydrogen isotopes such as $\mathrm{T}_{2}$, for which no experimental data are currently available. This method, described in Appendix A, has the significance that the Fredicted surface tension values are expected to have the correct temperature dependence, particularly in the region close to the critical point.

In Chapter $\mathrm{V}$ we comment on the limiting superheat temperature in liquid helium and its comparison with the maximum surface excess temperature corresponding to the peak heat flux conditions in steady-state heat transfer. 
We present arguments to show that such comparison is meaningless and the reported agreement between the two is largely fortuitous. We also point out that the spinodal can not be experimentally reached because of the more restrictive homogeneous nucleation in a superheated liquid.

\section{Effect of light on heat transfer}

In regard to our studies of transient heat transfer in liquid helium, we have discovered a new effect: a light• induced enhancement of transient heat transfer. We found that when the liquid helium in contact with the surface of our heater-thermometer is superheated up to a certain threshold temperature, the flashing of a light pulse of about 40 us duration on the solid-helium system produces a sudden large increase in the transient heat transfer. The experiment and the results are discussed in Chapter VI. This new effect seems to be quite significant because it offers the possibility of suppressing undesirable transient temperature excursions in the cryostatic stabilization of superconductive devices such as superconducting magnets. In addition to the possible technological value of this work, the discovery of the light effect suggests that it would be profitable to examine the effects of light on the initiation of nucleation; aiso, the effect may be a tool in studying electron bubbles in liquid helium. Furthermore, evidence that light affects the initiation of nucleate boiling has important implications for studies 
of nucleation rates and bubble growth processes which employ high speed photography and high intensity lighting as an experimental method, and also for any nucleation experiments in which glass Dewars or windows are used.

Transient heat transfer

Finally, in Chapter VII we present our study of the premature transitions to stable film boiling initiated by power transients in liquid nitrogen. We have shown for the first time in a cryogenic liquid that a transition to stable film boiling can be initiated for power values as low as about 40 per cent of the steady-state peak heat flux under transient heating conditions. This result may impose significant limitation in the design of cryogenic devices which are subjected to transients during operation or switchon in situations in which film boiling cannot be tolerated. We have also demonstrated how this premature transition can be inhibited by forced convection. Study of premature transitions to stable film boiling, besides being of technological importance, provides an excellent practical model for testing theories of bifurcation in nonequilibrium physical systems. 
CHAPTER II

TRANSIENT MAGNETORESISTIVE THERMOMETRY

IN LIQUID HELIUM

INTRODUCTION

There are many well known techniques for steady-state temperature measurements in liquid helium. However, there are situations in which it is necessary to accurately monitor fast changes in the temperature of the liquid helium in contact with a solid surface, and in these cases steady-state thermometric practices often prove to be inadequate. Two examples of situations in which transient techniques must be used are: (1) the study of transient heat transfer from solid surfaces into liquid helium, which has become very important with the recent interest in devices such as superconducting magnets and transmission lines, and (2) the study of nucleation phenomena in Iiquid helium. The interest in the latter area is twofold: first, to obtain information regarding the initiation of heterogeneous nucleation, and second, to determine the conditions under which metastability limits of superheated liquid helium are realized. With regard to heterogeneous nucleation, transient thermometry, in which the thermometer also acts as the heater, is particularly suitable for studying the 
inception of nucleation in liquid helium because, once the temperature of the liquid in contact with the solid surface has been measured, the temperature profile in the superheated liquid layer can be accurately determined from theory (Cole 1956, Schrock and Perrais 1966). In addition, the transient temperature response of the thermometer can provide useful information regarding activation and deactivation of nucleation sites on the thermometer-heater surface. Regarding homogeneous nucleation, transient thermometry is a very essential tool in determining the metastability limits of a superheated liquid because the limiting superheat temperature of liquid helium can only be realized under transient superheating conditions, with the possible exception of the region very close to the critical point.

In recent years, several transient techniques have been used with varying degrees of success to study the film boiling of liquid helium under transient heat transfer conditions. Such studies do not require a high degree of accuracy in the transient temperature measurements because only relatively large surface excess temperatures ( 20K) corresponding to film boiling are encountered. For instance, Jackson (1969) used a maganin wire as the heater-thermometer to study the transient film boiling in helium. Since the electrical resistivity of most metals and alloys becomes almost residual at liquid helium temperatures, the accuracy of temperature measurement was 
only of the order of $1 \mathrm{~K}$ in this experiment. Tsukamoto and Kobayashi (1975) used a thin lead film as the temperature sensor to detect the critical heat flux under pulsed heating conditions by the super-to-normal transition of the sensor. This study gives only one point at the superconducting transition temperature of the lead film, whereas the entire temperature development is desired. Iwasa and Apgar (1978) employed a gold-iron thermocouple attached to an indirectly heated copper surface to study the transient heat transfer in the film boiling regime. Due to the low sensitivity of the thermocouple at $4.2 \mathrm{~K}$ a transient superheat of less than $1 \mathrm{~K}$ could not be adequately studied. The temperature resolution reported in this work was only $0.25 \mathrm{~K}$ and the thermometer response was rather slow. However, recently Schmidt (1978) reported a heater thermometer for use in liquid helium with a response time in the microsecond region. A thin monofilimentary $\mathrm{NbTi} / \mathrm{Cu}$ compound conductor was used as a heater by applying a current greater than the critical current $I_{c}$ and the temperature was monitored via the temperature dependence of $I_{c}$. The temperature resolution was $\pm 0.1 \mathrm{~K}$, which is adequate for transient film boiling studies. All the thermometry techniques mentioned above are quite adequate for transient film boiling where large temperature differences occur and great accuracy in the temperature measurement is not imperative. However, in transient heat transfer studies below the critical power values, where 
primarily nucleate boiling is involved, the associated maximum superheat temperatures at $4.2 \mathrm{~K}$ are less than $0.4 \mathrm{~K}$ and it is desirable to have temperature resolution in the millikelvin range. None of the above mentioned techniques in their existing states is adequate under such conditions. Presently, there is only limited information available on transient heat transfer in liquid helium in the nucleate boiling regime, apparently because of a relative lack of accurate and sensitive thermometers which can be used for fast transient temperature measurements at such low temperatures. Desirable properties of such a thermometer include adequate sensitivity to millikelvin temperature changes, fast response time, convenient values of electrical resistance, low internal temperature difference, low Kapitza conductance, and reasonable ease of fabrication. Most of the above requirements are met by the carbon film thermometer used by Steward (1978) to study transient heat transfer over a wide range of applied powers so as to include both nucleate boiling and film boiling regimes. The heater-thermometer is comprised of a carbon film on a quartz substrate. Due to the small thickness of the film, typically 1000 \& (Sieward 1975), the response time of the film was extremely fast. The sensitivity was in the range of $\pm 10 \mathrm{mk}$. In spite of possessing most of the desirable properties of a transient thermometer, the carbon-film thermometer has several disadvantages which restricts its use to some extent. These 
include the need for a substrate, the low Kapitza conductance, $h_{k}$ of $0.7 \mathrm{~W} / \mathrm{cm}^{2} \mathrm{~K}$ at $4 \mathrm{~K}$ (Steward 1978), and the lack of a smooth heating surface. The low Kapitza conductance of carbon makes direct temperature measurement of the liquid helium in contact with the carbon film rather complicated unless properly corrected for at every step, which is a difficult process. In addition, the carbon film, due to its inherent particulate nature, offers numerous nucleation centers which may make the study of limiting superheat temperature in liquid helium particularly difficult.

In this chapter we describe a novel technique for measuring fast temperature excursions in liquid helium which is comparable in accuracy, sensitivity, and speed with the existing transient thermometry methods discussed above. In addition, this technique has particular advantages in measuring low transient superheat temperatures accurately over the entire helium I temperature range, from $2.2 \mathrm{~K}$ to $5.2 \mathrm{~K}$. This transient heater-thermometer is especially suitable for studying the initiation of heterogeneous nucleation and the limiting superheat temperatures in liquid helium and has all the other desirable properties of a fast transient thermometer. A brief summary of this transient thermometer has been previously presented (Brodie et al. 1978).

The thermometry technique we report here is adapted from our work on the galvanomagnetic effects in bismuth at 
liquid helium temperatures. Bismuth is a semi-metal that possesses the useful property that its electrical resistance increases by several orders of magnitude in an applied magnetic field at liquid helium temperatures. This magnetoresistive property of bismuth can be conveniently exploited as a thermometric property since the magnetoresistance of bismuth exhibits a strong temperature dependence at Iiquid helium temperatures.

A typical graph of the transverse magnetoresistance of a low quality single crystal of bismuth as a function of magnetic field at several bath temperatures is shown in Fig. 1. As can be seen from the figure, the electrical resistance increases from a zero field value of roughly $2 \mu \mathrm{ohm}$, to about $6 \mathrm{ohm}$ in a magnetic field (B) of about 1 Tesla. The oscillations in the curves are the characteristic de Haas - Shubnikov quantum oscillations which are periodic in 1/B. This oscillatory behavior in the magnetoresistance can be used advantageously. For instance, the temperature sensitivity of magneturesistance is the largest if one chooses a field corresponding to a peak in the curve. Also, if stability of the applied magnetic field is a problem, then an operating field can be chosen corresponding to a peak in the curve. Also, if stability of the applied magnetic field is a problem, then an operating field can be chosen corresponding to a peak in the curve where the variation of the resistance with the field is minimal. 


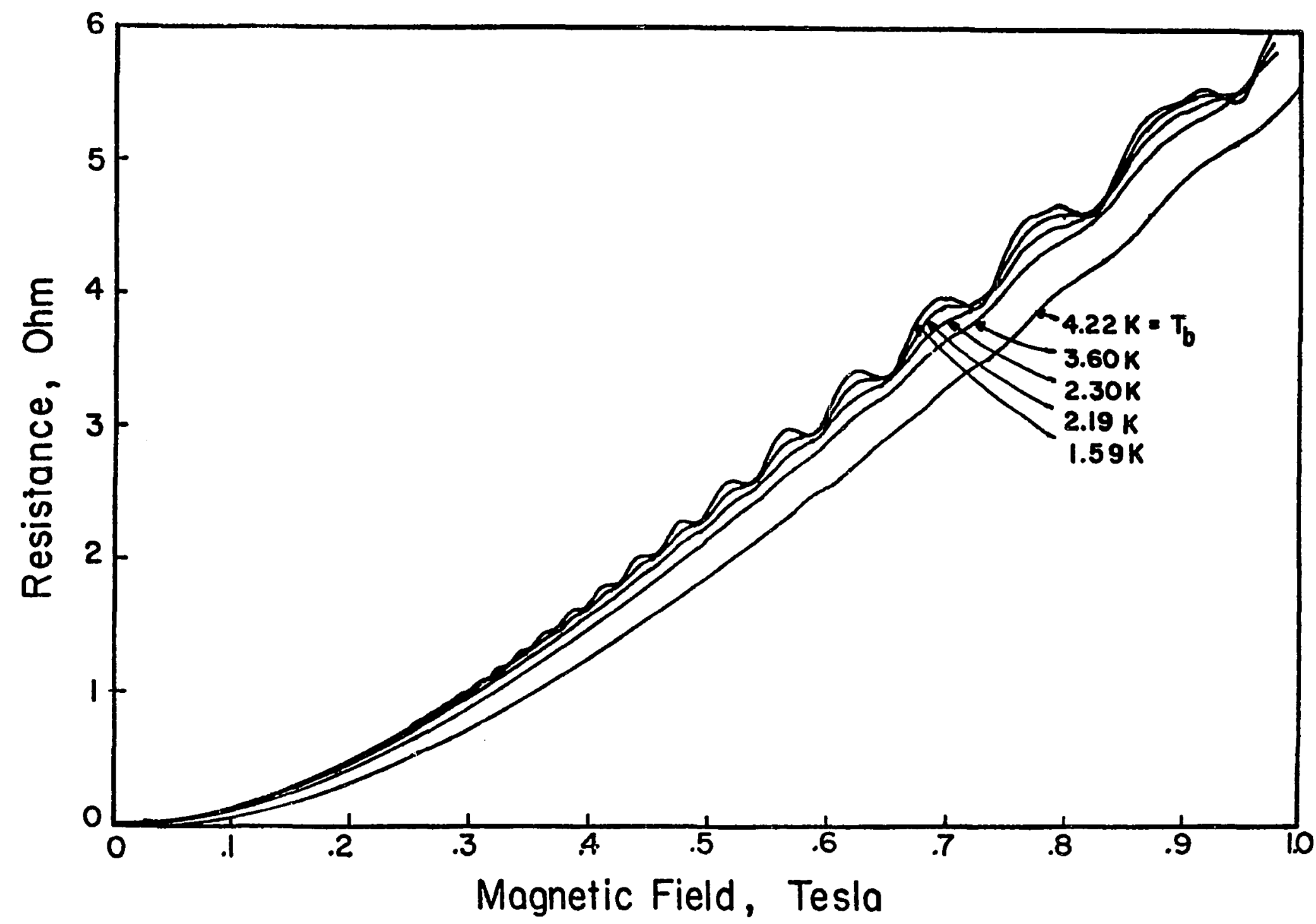

Figure 1. Transverse magnetoresistance of a single crystal of bismuth. Resistance of a single crystal of bismuth is shown as a function of the magnetic field at five different bath temperatures. The zero-field value is roughly $2 \mu \mathrm{ohm}$. 
The magnetoresistive thermometer can be used either in a passive mode in which the thermometer is used to measure the local bath temperature or in an active mode in which the thermometer is itself used as a heater through the Joule heating caused by the input of a time dependent current. It is for the latter case that the bismuth thermometer is most convenient, and the focus will be on the active mode of use. In the following sections we discuss the construction, calibration, and properties of a bismuth magnetoresistive thermometer.

\section{EXPERIMENTAL DETAILS}

\section{Thermometer construction}

Single crystals of bismuth were grown under a rough vacuum from 99.9998 purity bismuth in the shape of a rectangular parallelopiped 4-5 mm wide, about $1.5 \mathrm{~mm}$ thick, and 4-7 cm long. Other sizes and shapes could also be used to suit the conditions of a particular experiment. The crystals were grown by either normal freezing or zone melting techniques. In order to obtain higher purity and better temperature sensitivity the samples were recrystallized several times. Since bismuth expands upon freezing, it is necessary to use a 'soft mold' technique in which the melt is allowed to expand slightly as it solidifies. For the crystals described here, the mold was formed by pyrex glass plates piled together loosely. The glass surfaces were ground so as to prevent the bismuth from 
sticking to the surfaces. Although the crystals used in this work were oriented rather carefully with the crystalline trigonal axis perpendicular to the flat face and a binary axis perpendicular to the length, it is likely that other crystallographic orientations would also be satisfactory for thermometry.

The crystals were mounted in micarta holders, as shown in Fig. 2, shaped so as to support the crystal while allowing maximum exposure of the crystal surface to the liquid, especially in the middle where the potential leads of \#44 B\&S gauge copper wire were attached. Care was taken to fasten the crystal to the holder at only one point to avoid strain damage to the crystal due to differential expansion in liquid helium. The potential leads were attached to the bismuth crystal 8-9 mm apart by a spot welding technique in which the tip of the small wire is held in contact with the crystal surface while an electric current pulse is passed through both the wire and the crystal. The heated wire melts a small volume of bismuth directly under it, penetrates a short distance into the crystal, and is held fast when the bismuth solidifies. The larger size current leads were soldered to the ends of the crystal with bismuth solder. The holder and the crystal were positioned with long axis vertical in the liquid helium bath which is contained in the tail of a conventional pyrex double Dewar system, which is in turn located between the pole pieces of a 12-inch electromagnet. The direction of 


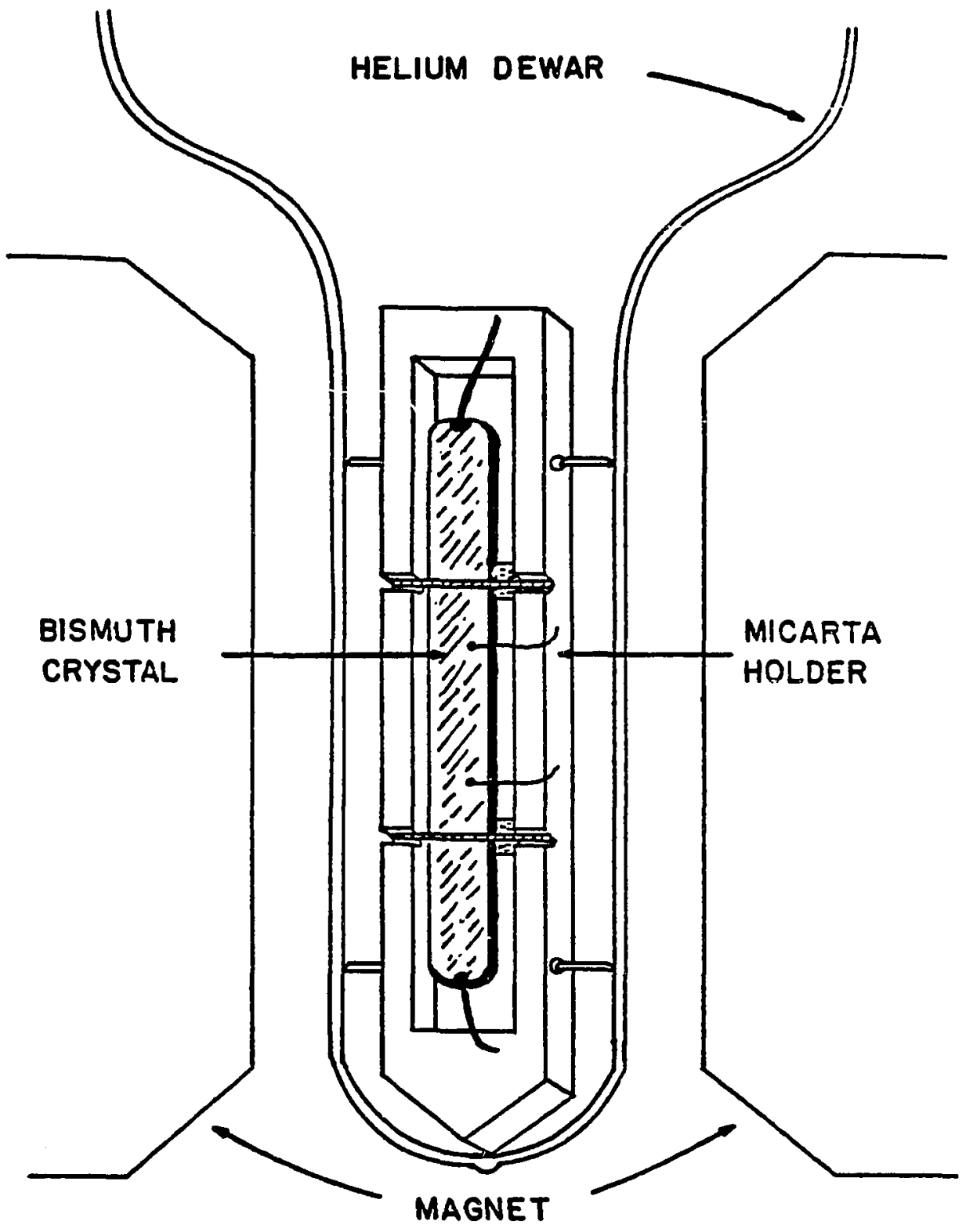

Figure 2. Experimental set-up. The thick lines at either end of the crystal indicate the current leads and the fine Iines at the center represent the potential leads. 
the magnetic field with respect to the crystal can be varied easily by rotating the magnet.

\section{Circuit details}

As mentioned previously, we discuss the case in which the bismuth crystal is being employed simultaneously as a heater and as a thermometer to measure the transient temperature response of the crystal helium bath system. We therefore assume that an arbitrary rate of Joule heating is achieved by the turning on of an appropriate heating current $I=I(t)$ in the crystal at time $t=0$. The circuitry described in this section is designed to monitor the transient temperature of the crystal-liquid helium system in response to the arbitrary rate of heating. Note that by limiting the current through the crystal to a very small value so as to preclude any self-heating of the crystal the thermometer can be employed passively to measure the bath temperature fluctuations. Thus it is useful to think of the following discussion as also including the passive usage.

A time-dependent electric current is applied starting at time $t=0$ and the change in the crystal temperature $\Delta T$ caused by the Joule heating is subsequently observed as a function of time $t$, employing the circuit shown in Fig. 3. The transient temperature response $\Delta T(t)$ of the crystal is easily determined by monitoring the temperature dependent change in magnetoresistance $\Delta R[T(t)]$ of the 


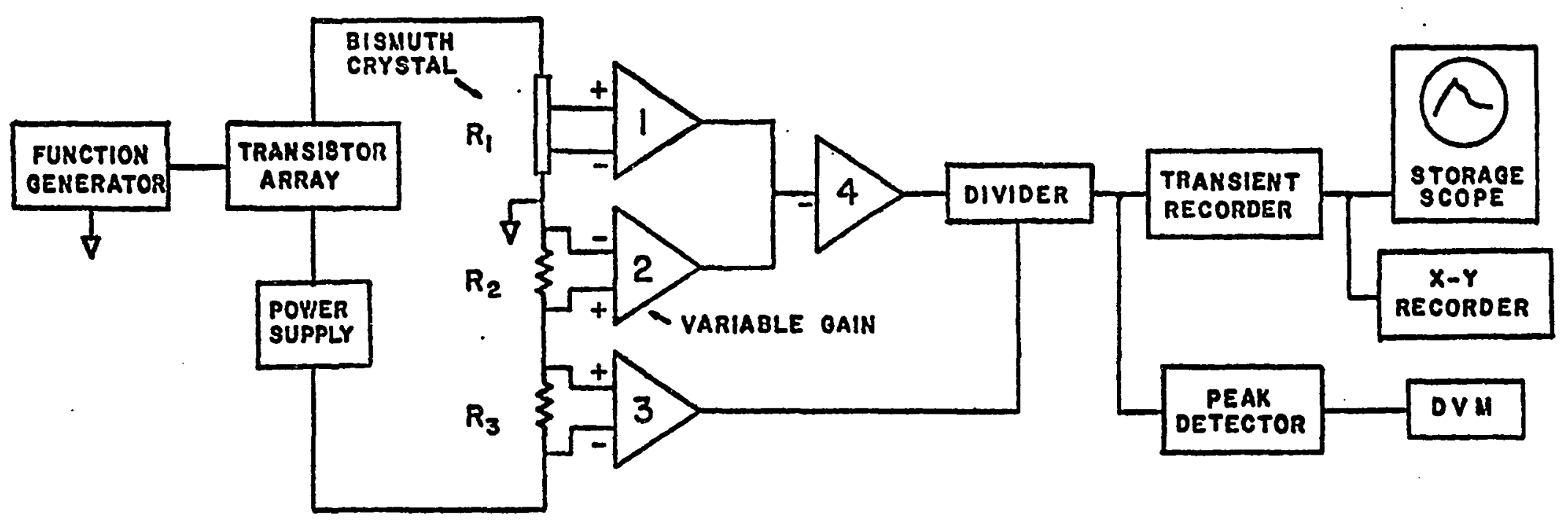

Figure 3. Schematic Circult diagram. 
crystal. The output from a function generator is fed to the transistor array so that a time-dependent current I(t) of any desired form can be delivered to the series circuit consisting of the bismuth crystal of resistance $R_{1}$ between the potential leads and the two resistors, $R_{2}$ and $R_{3}$, which have a low temperature coefficient of resistance. The crystal resistance $R_{1}$ depends on the applied magnetic field, the angular orientation of the crystal in the magnetic field, and the crystal temperature. Although our previous work (Brodie et al. 1977) has only involved the temperature response to a step-pulse heating current, we expect that other functions of current (e.g. ramp, exponential, etc.) can also be conveniently used. The voltages $R_{1} I(t)$ and $-R_{2} I(t)$, after amplification by the Analog Devices instrumentation amplifiers (AD 521) 1 and 2, are fed to the inverting input of the summing amplifier 4 (LF 356) to obtain the time-dependent voltage difference $\Delta V(t)$. These amplifiers have a gain bandwidth product of $40 \mathrm{MHz}$. If $\alpha$ and $\beta$ are the gains (typically $\sim 2$ ) of the amplifiers 1 and 2 we have for the duration of the current signal

$$
\begin{aligned}
\Delta V(t) & =\beta R_{2} I(t)-\alpha R_{1} I(t) \\
& =\beta R_{2} I(t)-\alpha\left[R_{1}(0)+\Delta R(t)\right] I(t),
\end{aligned}
$$

where $R_{1}(0)$ is the resistance of the crystal (between the potential leads) at the initial bath temperature. 
Initially, in the case of a step-pulse, the amplifier gain $B$ is adjusted so that $\Delta V(0)=0$ at $t=0$. This implies $\alpha R_{1}(0) I(0)=\beta R_{2} I(0)$ and we have

$$
\Delta V(t)=-\alpha \Delta R(t) I(t)
$$

for the duration of the current signal. An alternative way of making similar adjustments is to initially use a very small current so that there is no observable Joule heating of the crystal. Thus, we have $\Delta \mathrm{V}(t)=0$ and $\alpha R_{1}(0) I(t)=\beta R_{2} I(t)$ for $I(t)=0$ which ensures the same linear dependence of $\Delta V(t)$ on $\Delta R(t)$ as in Eq. (2), for all current values. This alternate adjustment procedure is a more general one and is applicable for all forms of current signals and not restricted to step-pulses. An Analog Devices AD 534 multifunction converter chip is used to divide the voltage difference $\Delta V(t)$ by the current signal from amplifier 3 (AD 521) to obtain a time-varying voltage which is proportional to $\Delta R(t)$. Since for small temperature changes $\Delta R$ is very nearly proportional to $\Delta T$ (for example, see Fig. 4), the amplification of the divider (AD 534) can be adjusted so that its output reads the superheat temperature $\Delta T(t)$ directly over a restricted temperature range. For a more accurate determination of temperature, reference is made to a calibration curve (see, e.g., Fig. 4). The temperature signal can either be fed directly to a storage oscilloscope or stored in a digital 
sampling type Biomation model 810 transient recorder for subsequent display on an oscilloscope or an $X-Y$ recorder. The output of the divider is also fed to a peak detector circuit which holds the peak value of the superheat temperature to be subsequently read by a digital voltmeter.

\section{Calibration}

The thermometer is calibrated by measuring the magnetoresistance as a function of the helium bath temperature over a temperature range of roughly $1.4 \mathrm{~K}$ to $4.6 \mathrm{~K}$. The bath temperature was controlled by varying the vapor pressure over the helium bath. The vapor pressure in the range above atmospheric pressure was monitored by a Setra System model 204 pressure gauge with a $4 \frac{1}{2}$ digit digital pressure indicator and the lower bath pressure below atmospheric was measured by mercury and oil manometers. The 1958 helium vapor pressure scale (van Dijk et al. 1960) was used to determine the bath temperatures.

The measurements of magnetoresistance were made using the standard four terminal method. The resistance measuring device was a $5 \frac{1}{2}$ digit Fluke model $8800 \mathrm{~A}$ digital multimeter. In the four terminal resistance measurement mode the instrument uses a current of $1 \mathrm{~mA}$ through the sample. This current produces power dissipation in the microwatt range which ensures no detectable heating of the bismuth crystal above the bath temperature. The input impedance of this device on the ranges used is $210^{9}$ ohms; 
this high input impedance allows this instrument to be used as a potentiometer so that essentially no current flows through the potential leads. The average of the resistance measurements with forward and reverse current through the crystal was taken at every bath temperature for calibration purposes. Figure 4 shows a typical calibration for an average quality bismuth crystal. For comparison, a calibration curve for the best quality crystal used is also shown alongside. The calibration curve is smooth with no noticeable bumps or wiggles in the entire temperature range. Above $4.2 \mathrm{~K}$ the calibration is practically linear which makes superheat measurements at atmospheric pressure particularly convenient.

\section{FACTORS AFFECTING THE TEMPERATURE SENSITIVITY OF THE CRYSTAL}

The temperature coefficient of magnetoresistance for a single crystal of bismuth at liquid helium temperatures is dependent upon three factors: the quality of the crystal, the strength of the magnetic field used, and the orientation of the crystal in the magnetic field. Therefore it is important to point out the effect of variations in these factors upon the temperature sensitivity of the crystal.

\section{Quality}

The quality of the crystal is judged by the high value in the ratio of zero field electrical resistance of the 

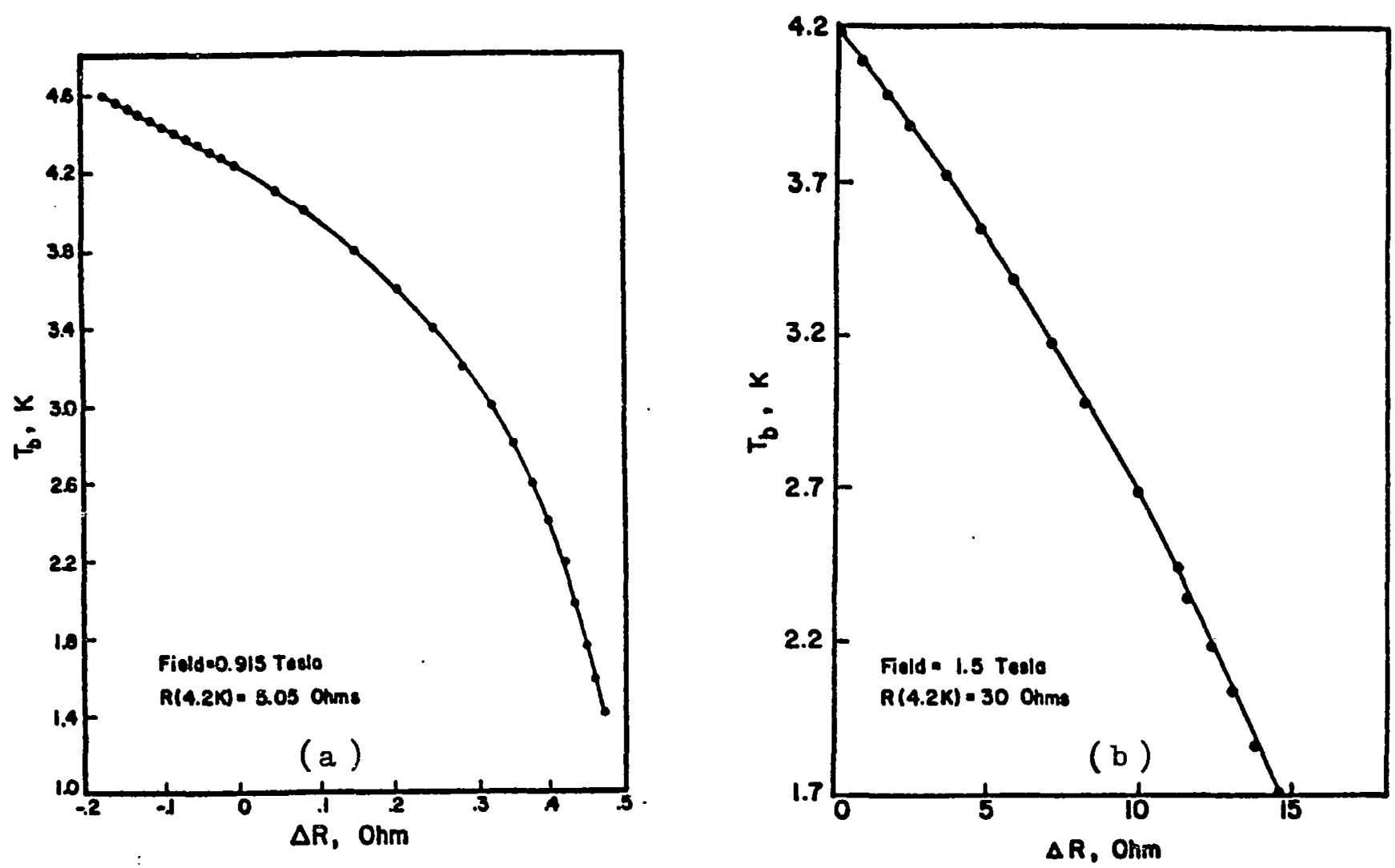

Figure 4. Calibration curve for bismuth crystals. The change in magnetoresistance $\Delta R$ as a function of the bath temperature (a) for an average quality crystal, and (b) for a crystal that has been recrystallized seven times. 
crystal at room temperature and at liquid helium temperatures. For the highest quality crystal used, which had been recrystallized several times $(\rho(300 \mathrm{~K}) / \mathrm{p}(4.2 \mathrm{~K}) \cong 500$ and $\rho(300 \mathrm{~K}) / \rho(2 \mathrm{~K}) \cong 1000$, the temperature sensitivity, $\mathrm{S}=\frac{1}{\mathrm{R}} \frac{\mathrm{dR}}{\mathrm{dT}}$, was $\sim .25$ at a magnetic field of $1.5 \mathrm{~T}$, bath temperature $4.2 \mathrm{~K}$, and with the crystalline trigonal axis parallel to the magnet field. The quality of a crystal is affected by mechanical strains associated with repeated thermal cycling between liquid helium temperature and room temperature, and also by careless physical handling. As an example, the sensitivity of the above crystal deteriorated from 0.25 to 0.23 over a 5 month period of active use. However, it is to be noted that improper physical handing of the crystal is more detrimental to the quality of the crystal than thermal cycling. For instance, for a crystal which was not moved from its position in the Dewar but subjected to several thermal cyclings (without the magnetic field on) between room temperature and $1.5 \mathrm{~K}$ over a period of several weeks, the temperature calibration was found to be constant within $\pm 3 \mathrm{mK}$ over the entire calibrated range. For best accuracy, however, it is advisable to recalibrate the bismuth thermometer each time it is used.

\section{Magnetic Eield}

The temperature sensitivity of the crystal is decidedly better at magnetic fields corresponding to the peaks in resistance vs. magnetic field curves as shown in 
Fig. 1. The amplitude of the de Haas-Shubnikov oscillations increases with decreasing temperature, probably rendering the crystal useful for temperature measurements even below $1.4 \mathrm{~K}$, which is the limit of our calibration. Although the absolute magnitude of the magnetoresistance decreases with decreasing magnetic field, the sensitivity, $S$, is not significantly reduced. Therefore, any magnetic field could be chosen corresponding to a peak in the R vs. B curve. The advantage of higher field is that the total change in the resistance $\Delta \mathrm{R}$ corresponding to a change in temperature at $\Delta \mathrm{T}$ is higher than that at lower magnetic field. For average quality crystals, which have been crystallized only once, the lowest workable magnetic field was found to be about $0.4 \mathrm{~T}$. An additional advantage of using an operating field corresponding to a peak in the R - B curve, besides that of greater temperature sensitivity, is that the effect of small fluctuations in the applied magnetic field on the calibration accuracy is minimal. The magnet used in our experiments is stable enough that no effect of field instability can be detected.

\section{Crystal orientation}

The direction of the magnetic field with respect to the crystallographic axes has a significant effect upon the temperature sensitivity of the bismuth thermometer. It is very essential to calibrate the thermometer with the magnetic field in the same direction as that for which the 
thermometer will be used. We have used only crystals oriented as stated earlier with the trigonal axes perpendicular to the flat face, and a binary axis normal to the length of the crystal. It is worth pointing out that for such a crystallographic orientation if the magnetic field is positioned parallel to the trigonal axis then a few degrees of rotation on either side from the normal does not significantly change the temperature sensitivity. For instance, $a \pm 2^{\circ}$ of rotation produces a calibration error of only $\pm 5 \mathrm{mk}$.

FEATURES OF THE BISMUTH THERMOMETER

The merits of the bismuth thermometer can best be judged by its performance in transient temperature measurements at liquid helium temperatures. As an example, we briefly describe here the results of our study of transient heat transfer and the limiting superheat temperatures in liquid helium I using the bismuth thermometer. Details of this particular experiment can be found elsewhere (Brodie et al. 1977). Figure 5 shows a typical set of superheat temperatures vs. time curves obtained from the step pulse heating of the bismuth heater-thermometer in liquid helium I for several values of applied power per unit area of the heater surface. The applied power values shown in the figure are in the range where the temperature overshoots develop sharp breaks at a superheat temperature $\Delta \mathbf{T}_{\mathrm{mc}}$ which is independent of applied power. At lower power 

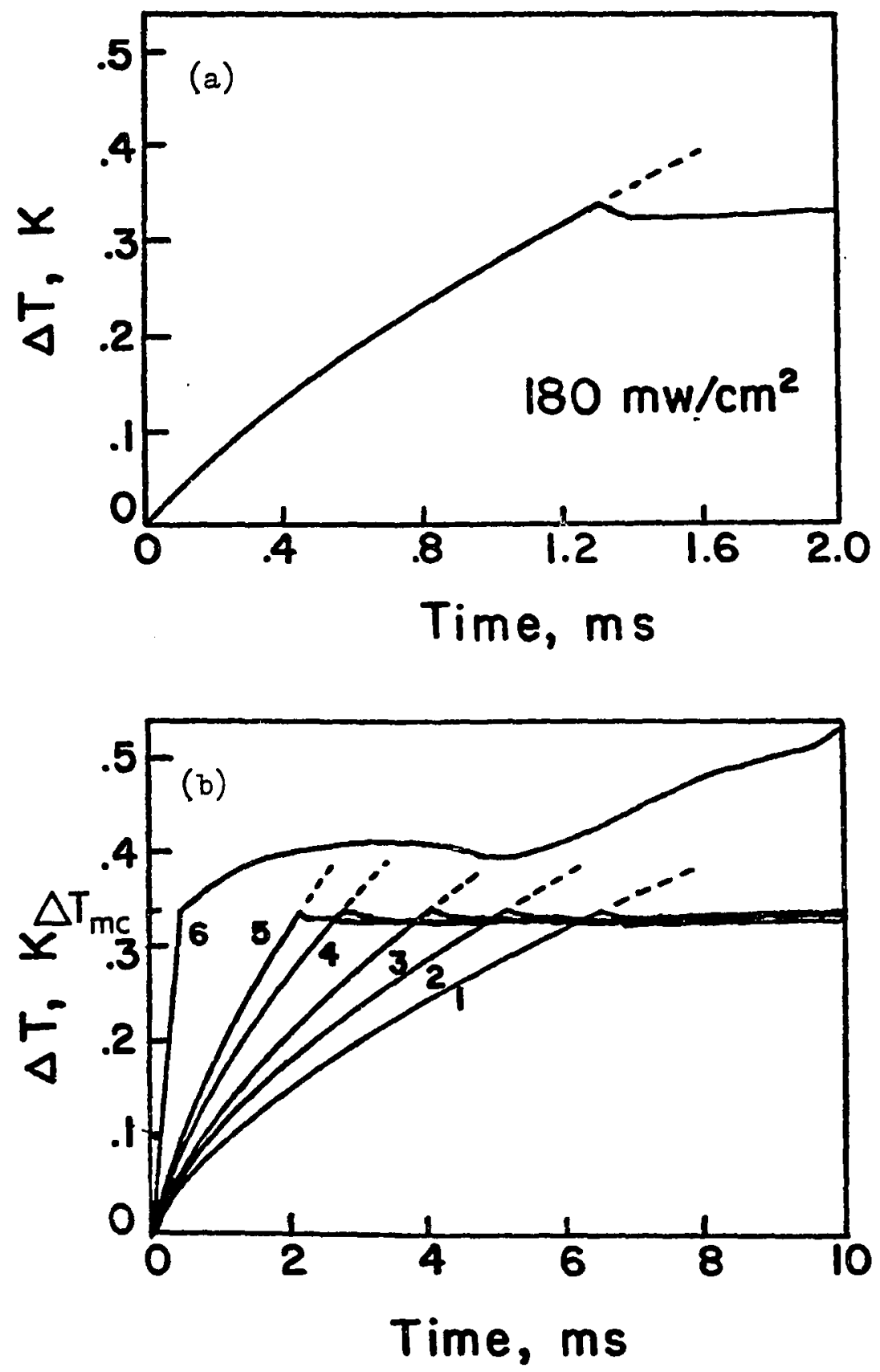

Figure 5. Superheat temperature $\Delta T$ vs. time. The bath temperature is $4.2 \mathrm{~K}$. The dashed Iines represent the predictions from the conduction equation. The numbers labelling each curve in (b) represent the applied power values s 1) 58,2$) 70,3) 80,4$ ) $106,5) 127,6) 430 \mathrm{~mW} / \mathrm{cm}^{2}$. 
values the overshoots are rounded in nature and their peak superheat temperatures depend on the applied power. Theoretical estimates of the homogeneous nucleation temperature based on the predictions from the classical nucleation theory show good agreement with the observed superheat temperature $\Delta \mathbf{T}_{\mathrm{mc}}$ (Brodie et al. 1977). The dashed lines in Fig. 5 represent the predicted timedependent superheat temperatures if it is assumed that the entire heat transport from the heater-thermometer surface into liquid helium is purely by thermal conduction. The expression for the conduction equation used is that of an infinite thin plate (Lurie and Johnson 1962). We now discuss the accuracy, response time, sensitivity, and the range of practical use of the bismuth thermometer.

\section{Accuracy}

A question of importance in transient thermometry concerns the accuracy of the temperature measurement of the liquid in immediate contact with the surface of the thermometer. This accuracy primarily depends on two factors: (1) the internal temperature difference, the difference between the measured average bulk temperature of the thermometer and the temperature of the solid surface in contact with the liquid, and (2) the Kapitza resistance which produces a temperature discontinuity $\Delta \mathrm{T}_{\mathrm{k}}$ between the surface of the thermometer and the liquid in contact. 
There are two indications that for the bismuth thermometer in Iiquid helium the experimentaliy measured average bulk temperature of the crystal and the temperature of the surface in contact with the liquid are very nearly the same. First, steady-state calculations show that for the entire range of applied powers used in our experiments the internal difference is less than $1 \mathrm{mK}$. This steady-state estimate should be a good approximation to the transient case for transient experiments whose time scales are slow compared to the thermal response time ( $~ 4 \mu s$, discussed later) of the crystal, a criterion which was met in our work. Second, as the bath temperature is lowered below the superfluid transition temperature, all indications of superheat as displayed by the $\Delta T$ vs. time curves arop by two or three orders of magnitude showing that the resistance change is negligible throughout the volume of the crystal and therefore that the efficient cooling due to the superfluid helium is transmitted almost undiminished to the crystal interior.

The second factor concerns the effect of the Kapitza conductance, $h_{k}$ From the Kapitza conductance data of Cheeke et al. (1973) for polycrystalline bismuth, a temperature difference $\Delta \mathbf{T}_{\mathrm{k}}$ of about $20 \mathrm{mK}$ can be estimated to exist between the solid's surface and the liquid helium in contact when the heat flux is $0.1 \mathrm{~W} / \mathrm{cm}^{2}$ and the bath temperature is $4.2 \mathrm{~K}$. However, this estimate may be too large for two reasons. First, the data of cheeke et al. 
(1973) are for a polycrystalline sample of bismuth which might be expected to have a smaller Kapitza conductance than our single crystals of bismuth (Frost 1975). Second, there is evidence that the Kapitza conductance is increased by the application of a magnetic field (Challis and Sherlock 1969). This effect is thought to be due to a reduction in the density of electrons at the surface states by the application of a magnetic field. As a result of this, the phonon-electron scattering at the surface is reduced and the Kapitza conductance is increased. Thus $\Delta \mathrm{T}_{\mathrm{k}}$ for a single crystal of bismuth at a representative heat flux value of $0.1 \mathrm{~W} / \mathrm{cm}^{2}$ may be smaller than $20 \mathrm{mK}$. Fortunately, the actual error due to Kapitza conductance in the temperature measurement with the bismuth thermometer is significantly lower than that mentioned above because of the differential electronic technique used to monitor the transient superheat temperature. In the transient heating case, for all practical purposes, the temperature difference $\Delta \mathrm{T}_{\mathrm{k}}$ due to Kapitza conductance can be considered separately since the time domain $\tau$ in which Kapitza conductance is dominant, the first few microseconds, is well separated from the domain in which thermal conduction prevails. As a result of this, $\Delta T_{k}(t)_{t}=\tau$ appears as an offset superimposed on $\Delta T_{f}(t) t>\tau^{\prime}$, the superheat temperature of the fluid. Thus $\Delta T_{k}(t){ }_{t=\tau}$ can be effectively subtracted out by the differential adjustment technique described earlier, and consequently only $\Delta T_{f}(t)$ is observed. The residual 
error due to the change in the Kapitza conductance as a function of temperature during superheating is estimated to be less than $\pm 5 \mathrm{mK}$. This latter fact is confirmed by the excellent agreement of the conduction equation, dashed line in Fig. 5, with the experimentally observed superheat in the initial stages of superheating when the heat transfer mechanism is predominantly thermal conduction. Note that the conduction equation used does not take into account the effect due to Kapitza conductance.

It is interesting at this point to compare the Kapitza conductance value of the carbon-film thermometer which has been previously used for similar transient heat transfer studies (Steward 1978). The reported value of Kapitza conductance associated with the carbon thermometer is an order of magnitude lower than the value for the polycrystalline bismuth sample. Moreover, the change of $h_{k}$ with temperature is also proportionately larger. Since it is difficult to exactly correct for the temperature due to Kapitza conductance it may be convenient for accurate temperature measurements to use a thermometer in which this error is very small to begin with. The overall estimated uncertainty in the transient temperature measurement with the bismuth magnetoresistance thermometer is $\pm 10 \mathrm{mK}$.

\section{Response time}

An important feature of the bismuth thermometer, the fast response time, is clearly evident from the superheat 
temperature vs. time curves shown in Fig. 5. Notice in particular the sharp breaks in the temperature overshoots which would not have been produced unless the response time of the thermometer were on the order of $100 \mathrm{\mu s}$.

In fact, the estimated response time is less than $100 \mu \mathrm{s}$. In making a theoretical estimate of the response time of a transient heater-thermometer, there are two factors which must be considered: (1) the time, $\tau$, for the temperature changes inside the thermometer to relax to the steady-state distribution, and (2) the time, $\theta$, for the thermometer to respond to rapid temperature fluctuations of the liquid in contact with the thermometer surface.

The time $\tau\left(\tau=d^{2} / 4 \alpha\right.$, where $d$ is the thickness and a the thermal diffusivity of the crystal) for the bismuth thermometer is of the order of $4 \mu \mathrm{s}$, which can be further shortened by reducing the thickness of the crystal. There is evidence that the thermal conductivity of bismuth is primarily due to phonon transport at liquid helium temperatures (Kuznetsov et al. 1970, Kopylov et al. 1971, Korenblit et al. 1970) and therefore should only be weakly affected by the magnetic field (Bhagat and Manchon 1967, Klemens 1956). Thus in the case of bismuth the value of $\tau$ is not likely to be strongly magnetic field dependent. The time $\theta \quad\left(\theta=\frac{\rho V C}{h_{k} A}\right.$, where $V$ is the volume, $\rho$ the density, $c$ the specific heat, $h_{k}$ the Kapitza conductance, and $A$ the surface area of the thermometer), in the active 
mode of use, for the bismuth thermometer is of the order of $90 \mu \mathrm{s}$ which is significantly greater than $\tau$. However, this estimate of $\theta$ is likely to be too large since it is based on a value of $h_{k}$ for a polycrystalline sample of bismuth which may be exceeded by our single crystals in a magnetic field as discussed earlier. The overall response time of the thermometer is essentially the larger of $\tau$ or $\theta$.

\section{Sensitivity}

The sensitivity, $S=\frac{1}{R} \frac{d R}{d T}$, of the bismuth thermometer compares very well with that of the carbon-film thermometer. At $4 \mathrm{~K}$ it varied from $0.25 \mathrm{~K}^{-1}$ for the best crystal used down to $0.1 \mathrm{~K}^{-1}$ for an average quality crystal. These values of sensitivity are representative of all the bismuth crystals used with varying qualities and magnetic fields ranging from about $0.4 \mathrm{~T}$ to $1.5 \mathrm{~T}$. In comparison, the sensitivity for a carbon-film thermometer at $4 \mathrm{~K}$ is about $0.1 \mathrm{~K}^{-1}$ (Shen and Heberlein 1972).

\section{Range}

We have used the bismuth heater-thermometer in liquid helium from about $1.4 \mathrm{~K}$ to $4.8 \mathrm{~K}$. The sensitivity at $1.4 \mathrm{~K}$ is still quite adequate and consideration of the appropriate thermophysical properties of bismuth suggests that this range might be extended down to about $1 \mathrm{~K}$. Below this temperature the Kapitza conductance may become objectionably low, the internal temperature difference too large and the 
sensitivity too small. The range on the high side should also extend above $4.8 \mathrm{~K}$ into the liquid hydrogen region, being limited only by the increasing response time.

Specific applications and advantages

An important application of the bismuth thermometer is in determining the limiting superheat temperatures of liquid helium. It is particularly well suited to this use because, besides having millikelvin accuracy and fast response time, the bismuth crystal can readily be electropolished so as to present a smooth surface to the liquid helium. This is a matter of significance because it is necessary to have a smooth surface, i.e. to have a small number of heterogeneous nucleation sites, in order to be able to superheat the liquid helium up to its limiting superheat temperatures without the disturbing influence of heterogeneous nucleation. Unfortunately, in the case of carbon-film thermometer it is likely that there is a large number of potential nucleation sites on the carbon surface. This may be a reason that steward (1978) fails to observe a sharp peak or kink in the temperature overshoots corresponding to the limiting superheat temperature of liquid helium, in contrast to the present work.

An important advantage of the bismuth thermometer is the ease with which its electrical resistance can be altered to suit the requirements of a particular situation. At liquid helium temperatures the magnetoresistivity of 
a single crystal of bismuth can be increased by six or seven orders of magnitude above a zero field value of about $2 \times 10^{-7}$ ohm-cm by increasing the magnetic field from zero to one or two Tesla. Thus, the bismuth crystal heater resistance can be easily matched to the power source. A related advantage of the bismuth thermometer is that it is possible to obtain almost any desired resistance vs. temperature characteristic simply by varying either the magnitude of the applied magnetic field or the orientation of the crystal in the magnetic field.

There is another advantage of the bismuth thermometer which could be very useful in constant current heat transfer studies in liquid helium. Because of the large negative temperature coefficient of magnetoresistance of bismuth, the temperature rise which occurs with the nucleate to film boiling transition will tend to be self-regulating due to the lowering of the applied power to the crystal. Thus there is reduced danger of heating element burn out. 
CHAPTER III

\section{CORRESPONDING STATES BEHAVIOR OF IIQUID-TO-VAPOR NUCLEATION IN CLASSICAL AND QUANTUM LIQUIDS}

\section{INTRODUCTION}

The problem of nucleated phase changes in metastable fluids has been an active area of study for many years. Much of the effort in this area, both experimental and theoretical, has been directed toward the understanding of the liquid-to-vapor nucleation in superheated liquids and on the complementary problem of vapor-to-liquid condensation process in supersaturated vapors. In studies of nucleation in superheated liquids, the experimental goal has been to determine the homogeneous nucleation temperature for liquids (Blander and Katz 1975, Blander 1979, skripov 1974). The theoretical effects are concerned with predicting such homogeneous nucleation superheats for liquids on the basis of homogeneous nucleation theory which provides the rate of formation of vapor bubbles in a superheated liquid without the effects of solid surface, impurities or high energy radiation. The homogeneous nucleation theory developed primarily by Becker and Doring (1935), Volmer (1939), Zel'dovich (1943), and Frenkel (1946) is commonly referred to as the 'classical' nucleation theory. 
Predictions from the BDVZF nucleation theory are generally in excellent agreement with the experimental limiting superheat temperatures of a large number of organic liquids (Blander 1979, Skripov 1975) and several cryogenic liquids (Skripov et al. 1979) .

It is worth pointing out in this context that such agreement between theory and experiment is not always observed in the case of the complementary problem of vaporto-liquid condensation process in supersaturated vapors. Consequently, doubts have been expressed regarding the validity of the classical nucleation theory, at least in the case of condensation process and it has even been assumed that a satisfactory nucleation theory does not yet exist.

A knowledge of the liquid-to-vapor nucleation process in superheated liquids is fundamental to a basic understanding of a number of important practical problems, including the vapor explosion phenomena. Besides, such knowledge is essential in defining conditions under which metastable liquids can be maintained for performing physical measurements. Also, such measurements would partly span the gap between our knowledge of dense gas and our as yet incomplete understanding of liquids.

To this date, little attention has been directed toward the study of simple fluids such as the noble gases and to an examination of the quantum effects that may possibly influence the nucleative behavior of these fluids. 
In this connection it is worth mentioning that the tensile strength experiments in liquid helium have yielded results in complete disagreement with the predictions from the classical nucleation theory. Beams (1959) points out that such disagreement may be due to the fact that the nucleation theory might not be expected to hold for liquid helium. Marston (1976) asserts that either the nucleation theory fails to describe homogeneous nucleation in superfluid helium, or the experimentally observed cavitation did not nucleate from fluctuations. Marston further speculates that the production of voids may be significantly different in quantum Iiquids because the zero-point atomic motion is significant in comparison with the thermal motion. Leaving aside the inherent uncertainties involved in the cavitation strength experiments, the observed lack of agreement with theory may be explained on the basis of the following arguments: (1) the classical nucleation theory is not valid for a quantum liquid such as liquid helium, (2) the nucleation theory applicable to the case of helium should be modified due to the possible quantum effects which may influence the nucleative behavior of liquid helium, and (3) an entirely different mechanism of nucleation is involved, such as nucleation due to quantum tunneling of the thermodynamic barrier as suggested by Lifshitz and Kagan (1972).

The purpose of this chapter is threefold. First, to test the validity of each of the above three arguments 
by taking an altogether different experimental approach from the usual tensile strength experiments. Second, to discuss homogeneous nucleation temperature measurements in ${ }^{4}$ He. Third, to examine aspects of nucleation in classical and quantum liquids from the viewpoint of corresponding states.

\section{THEORETICAL BACKGROUND}

Nucleation in a liquid-vapor system constitutes a collective process in which the system undergoes a transition between two regions of phase space separated by an energy barrier. In the special case in which the system undergoes this transition along a configurational path of minimum energy and when the nucleus is much larger in size than a small molecular cluster, it is often useful to approximate it as a sphere of radius $r$. One then assumes that the energy barrier profile is represented by the form

$$
W(r)=A r^{2}-B r^{3}
$$

where the first and second terms represent the usual surface and bulk contributions to the work of formation of the nucleus (Abraham 1974, Uhlman and Chalmers 1967). The quantities $A$ and $B$ involve the surface tension and latent heat which are set equal to their flat interfacial surface value (the capillarity approximation) and infinite bulk value, respectively. In this simplified picture the 
complexities of phase space coordinates are replaced by the single collective coordinate $r$ and the details of molecular dynamics by bulk thermodynamics.

Whether or not one chooses to accept these approximations, it is possible to distinguish two processes by which passage from one side of the barrier to the other side can be accomplished: Nucleation can take place either over the barrier or through the barrier. In the first case, i.e. thermally activated nucleation, it is thermal fluctuations that drive the system over the energy barrier. In the second case the system may penetrate through the barrier through a purely quantum mechanical tunneling process (quantum tunneling nucleation). The latter case is analogous to the process of impurity atom tunneling between sites in a solid.

Thermally activated processes (e.g. nucleation) occurring at temperature $T$ are characterized by a steadystate expression of the Arrhenius type

$$
J=J_{0} \exp \left(-W_{A} / k T\right)
$$

where $J$ is the nucleation rate, i.e. the number of macroscopic nuclei formed per unit time and volume out of the metastable phase, $W_{A}$ is the height of the energy barrier (3.1), and represents the reversible work required in the formation of a critical nucleus. Furthermore, $k$ is the Boltzman constant, and $J_{0}$ is the attempt frequency. 
A thermal activation rate expression of the form of Eq. (3.2) is encountered in a wide range of contexts, including chemical reaction kinetics, and can be derived on very general grounds (Kramers 1940, Landauer and Swanson 1961, Binder and Stauffer 1976). In the case of thermally activated nucleation, expressions of the form (3.2) are obtained within the traditional Becker-Doring-VolmerZel'dovich-Frenkel (BDVZF) theory or, more generally, within formulations such as the master equation (Binder and stauffer 1976). The traditional BDVZF theory employs a chemical kinetics reaction chain in which monomers successively collide with $i$-mers to form nuclei of critical size which then can grow spontaneously. The reaction chain is of the form

$$
\mathrm{A}_{\mathrm{i}}+\mathrm{A}_{1} \neq \mathrm{A}_{\mathrm{i}+1}
$$

where $i \geq 1$. One then solves for the rate of formation of critical size nuclei under quasi-steady-state assumptions (see the recent review by Abraham 1974). We employ the BDVZF nucleation rate expression in the form

$$
J=N\left(\frac{2 \gamma}{\pi m B}\right)^{\frac{1}{2}} \exp \left[\frac{-16 \pi \gamma^{3}}{3 k T\left(P_{e}-P_{L}\right)^{2} \delta^{2}}\right]
$$

which is derived from (3.2) and (3.3). The specific form of the exponential term, which represents the ratio of the minimum work required to form a critical nucleus $w_{c r}$ to 
the mean energy of thermal motion per degree of freedom of the system kT, is obtained from macroscopic thermodynamic considerations. In expression (3.4), $J$ is the rate of formation of critical nuclej. per unit volume and time, $\gamma$ is the surface tension of a flat liquid-vapor interface, $\mathrm{N}$ is the molecular number density of the liquid, $\mathrm{m}$ is the molecular mass, $B$ is a constant $(B=2 / 3), \mathrm{P}_{e}$ is the equilibrium vapor pressure, and $\delta$ is the Poynting correction:

$$
\delta=\left(P_{V}-P_{L}\right) /\left(P_{e}-P_{L}\right)
$$

which corrects for the fact that $\mathrm{P}_{\mathrm{V}}$ is the vapor pressure of the liquid under an ambient pressure, $\mathrm{P}_{I}$, whereas the equilibrium vapor pressure $P_{e^{\prime}}$ is measured with the liquid at an ambient pressure, $\mathrm{P}_{e}$. It is worth noting here that for a liquid in which the contact angle is zero (e.g. liquid helium), the nucleation temperature, $T$, determined from (3.4) is insensitive to the presence of a solid surface (Fisher 1948, Cole 1974).

A question of central importance to this paper is whether the rate expression (3.2) (or, more specifically, Eq. (3.4)) is valid for a guantum mechanical fluid such as ${ }^{4}$ He. From a theoretical standpoint, one would expect that the answer to this question is yes as long as the process is one of thermally activated nucleation. The claim that the BDVZF rate expression should be quantum 
mechanically valid can be argued from its derivation based on the reaction chain of Eq. (3.3). The chemical kinetic rate expressions obtained from reaction chain (3.3) should be quantum mechanically valid in exactly the same sense and to the same degree that, for example, the chemical rate expressions that one can write based on the hydrogen reactions $\mathrm{H}+\mathrm{H} \rightleftharpoons \mathrm{H}_{2}$ are quantum mechanically valid. As discussed in the following sections of this chapter, we do find experimentally that Eq. (3.4) correctly predicts the homogeneous nucleation temperature of ${ }^{4} \mathrm{He}$. (Because of the extreme sensitivity of the nucleation rate $J$ on the temperature of the superheated liquid, one adopts a more or less conventional usage of referring to a single temperature, the homogeneous nucleation temperature.)

Relatively little work has been directed specifically toward the question of liquid-to-vapor transitions via a quantum tunneling process. The question was examined by Lifshitz and Kagan (1972) who proposed a quantum mechanical model for the nucleation process. Their model effectively reduces to the problem of a position-dependent virtual nucleus of the vapor phase tunneling through an energy barrier of the form (3.1) which separates the initial metastable state of the liquid from the state with the real nucleus. The position coordinate in their model corresponds to the radius of a spherical nucleus and constitutes a single collective variable for the problem. The LifshitzKagan analysis would indicate that quantum tunneling 
nucleation should predominate in metastable liquids only at temperatures less than a temperature $T_{0^{-}}$For metastable fluids at temperatures above $T_{0}$, the analysis indicates that the predominant liquid-to-vapor phase change process is thermally activated nucleation and that the rate expression is of the form $(3.2)$. We estimate the temperature $\mathbf{T}_{0}$ from the following expression given by Lifshitz and Kagan (1972)

$$
T_{0}=\left[\frac{256}{135 \sqrt{2} \pi k} \frac{h^{2}}{m a_{1}{ }^{2}}\left(\frac{\gamma a_{1}^{2}}{h^{2} / m a_{1}{ }^{2}}\right)^{\frac{1}{2}} \frac{P_{1}}{\left|P_{1}-P_{2}\right|}\right]\left(\frac{a_{1}}{R_{0}}\right)^{\frac{3}{2}}
$$

to be approximately $0.3 \mathrm{~K}$ for liquid ${ }^{4} \mathrm{He} . \mathrm{R}_{\mathrm{O}}$ is the width of the energy barrier. The notation employed in expression (3.6) is that of Iifshitz and Kagan (1972). Lifshitz (1975) cites an estimate of about $1 \mathrm{~K}$. Taking either estimate of $T_{0}$ metastable liquids at these temperatures are unlikely to homogeneously nucleate except in the negative pressure region of the liquid-vapor phase diagram and therefore can only be studied in experiments involving tensile strength or cavitation. Our superheating measurements reported in this work were carried out at homogeneous nucleation temperatures of about $4 \mathrm{~K}$ and above. We therefore rule out quantum tunneling nucleation as a contributing mechanism in our studies, and we adopt Eq. (3.4) as the appropriate rate expression. 
EXPERIMENTAL TECHNIQUES

In examining the liquid-to-vapor nucleation phenomenon there have been two classes of experiment: superheating, and tensile strength or cavitation. This chapter will primarily focus on the superheating type of experiment. In superheating experiments the objective is to reach the maximum attainable superheat beyond which the homogeneous nucleation process renders further superheating impossible. This maximum can be approached in two ways: (1) by quickly expanding a given volume of liquid as is done in bubble chambers or (2) by direct superheating of a small amount of liquid, usually in droplets, at constant pressure. There are several ways the latter process can be accomplished.

Earlier data available from bubble chamber studies (Bugg 1959) are not reliable for quantitative comparisons with the homogeneous nucleation theory. However, in the recent years, specially designed bubble chambers for studying nucleation have yielded results in good agreement with theory. For example, Sinitsyn and Skripov (1968) and Skripov (1974) report nucleation measurements for several organic liquids which are in excellent agreement with the BDVZF nucleation theory. No such systematic bubble chamber measurements for cryogenic liquids, particularly for liquid helium, are currently available. However, Hord et al. (1964) report bubble chamber studies in nitrogen and 
hydrogen. Although their data show a large scatter, the highest superheat values in the case of liquid hydrogen are found to be consistent with the predictions from the nucleation theory.

\section{Exploding aroplet theory}

Among the direct superheating methods, the exploding droplet technique has received the most attention due to its simplicity and has most often been used to study the homogeneous nucleation temperatures of a large variety of organic liquids (Moore 1959, Wakeshima and Takata 1958, Porteus and Blander 1975, Blander et al. 1971, Skripov and Sinitsyn 1964, 1967, Sinitsyn and Skripov 1968, Skripov and Ermakov 1964). In this technique an involatile medium immiscible with and denser than the fluid to be studied is externally heated in a column so that it is hotter at the top than the bottom. The volatile liquid to be studied is introduced at the bottom of the column in many cases simply by using a hypodermic syringe. The small droplets of the volatile liquid become superheated as they rise slowly in the column and when the superheat reaches a value where the nucleation rate becomes significant, the droplets explode with a sharp report. The temperature at which they explode is measured easily with thermometers set near the levels where the droplets nucleate explosively. Despite the inherent simplicity, this method cannot be used for measuring the homogeneous nucleation temperature of a 
cryogenic liquid such as liquid helium, the main problem being that no appropriate host liquid exists for use at such low temperatures.

\section{Pulse heating method}

Skripov and Pavlov (1970) and Pavlov and Skripov (1965, 1967, 1970) have introduced a new technique by which the homogeneous nucleation temperature can be determined in a solid/liquid system, i.e. even in the presence of heterogeneous nucleation sites. This is accomplished by pulse heating a thin platinum wire submerged in a liquid; if the heating rate is sufficiently rapid, about $10^{6} \mathrm{k} / \mathrm{sec}$, the liquid in contact with the wire can be superheated sufficiently to nucleate explosively. During rapid heating, the available nucleation sites on the wire can only dissipate part of the internally generated heat due to the finite growth rate of the bubbles formed. Consequently, the liquid in contact with the wire can become highly superheated before the bubbles have a chance to coalesce and form a vapor blanket. Recently, the author (Brodie et al. 1977) independently developed a similar transient superheating technique for determining the homogeneous nucleation temperature of liquid helium I. This technique is described below.

Transient superheating technique in helium

We have found that liquid helium in contact with the surface of a solid can be superheated up to its homogeneous 
nucleation temperature by pulsing an electric current through the heater. Such superheating becomes possible primarily due to the finite delay time involved in the inception of heterogeneous nucleation at the solid-liquid interface. This delay time is of the order of 30-100 milliseconds and is found to be dependent on the applied power to the heater, decreasing in time with increasing power. Therefore, if the heating rate is sufficiently high, the homogeneous nucleation limit is reached before appreciable heterogeneous nucleation can take place. The existence of such a delay time in cryogenic liquids has been reported by Oker and Merte, Jr. (1978). The delay time, particularly in liquid helium, is due to its excellent wettability which makes the presence of vapor-filled sites on a submerged solid surface very unlikely. Moreover, at such low temperatures any dissolved impurity gases will be frozen. Consequently, under transient heating conditions, in the initial periods the superheating of the liquid in contact with the heater surface is entirely due to thermal conduction. Heterogeneous nucleation does not occur until an appropriate superheat and thermal boundary couditions are reached (Hsu and Graham 1971, Han and Griffith 1965). Due to the long delay times involved, the heating rate needs to be only of the order of $10 \mathrm{k} / \mathrm{sec}$, in contrast with the extremely high heating rate $\sim 10^{6} \mathrm{~K} / \mathrm{sec}$ required in the pulse heating technique of Pavlov and skripov mentioned previously. We discuss below the details of the methodology 
of this transient superheating technique and also point out the differences with the pulse heating technique. In the pulse heating method, Pavlov and Skripov (1965, 1967, 1970) used a thin platinum wire as the heaterthermometer in their studies of the homogeneous nucleation in a number of organic liquids. However, in the case of liquid helium, conventional resistive thermometry employing metals and alloys become quite ineffective and inadequate for temperature measurements under transient heating conditions primarily because of their extremely low temperature coefficient of resistance at such low temperatures. Consequently, we have developed a new magnetoresistive thermometry technique which is convenient and accurate for simultaneous heating and temperature measurement under transient conditions in liquid helium (Brodie et al. 1979, Chapter II). We had used this technique to study transient heat transfer and to make preliminary measurements of the homogeneous nucleation temperature in liquid helium (Brodie et al. 1977).

Our investigations involve the step-pulse heating of a single crystal of bismuth immersed in a bath of liquid helium I. A constant electric current is applied at $t=0$, and the change in crystal temperature caused by the Joule heating is subsequently observed as a function of time. The superheat temperature of the crystal is relatively easy to determine because of the strong temperature dependence of magnetoresistance of a high quality bismuth crystal 
at liquid helium temperatures. Thus, the crystal can be used as its own thermometer when properly calibrated. The crystals used were in the shape of parallelopiped and were as large as 4-7 cm long, $1.5 \mathrm{~mm}$ thick and 4-5 mm wide. The electronic circuitry and experimental details were presented in chapter II.

\section{OBSERVATIONS}

Typical superheat temperature $\Delta T$ vs. time curves are shown in Figs. $6 a$ and $6 b$ for several values of applied power per unit area of the solid's surface, P. The superheat temperature as a function of time initially rise above, i.e. overshoot, their steady-state values. Each curve subsequently cools toward its steady-state value after attaining a maximum superheat temperature $\Delta \mathrm{T}_{\mathrm{m}}$. For the relatively lower range of values of $\mathrm{P}$ (Fig. 6a) the temperature overshoots appear to be smoothly rounded in nature. As $\mathbf{P}$ is increased, the maximum superheat temperature $\Delta \mathrm{T}_{\mathrm{m}}$ of these overshoots becomes greater in magnitude and occurs at increasingly earlier times.

At somewhat higher values of $\mathrm{P}$, typically above $30 \mathrm{mw} / \mathrm{cm}^{2}$, the temperature overshoots have two distinct characteristics: (1) the transient superheat curves develop quite sharp peaks at the maximum temperature, and (2) the maximum superheat temperature $\Delta T_{\mathrm{m}}$ becomes a constant $\Delta \mathrm{T}_{\mathrm{mc}}$ (within about $5 \mathrm{mk}$, the resolution of our experiment), independent of the applied power P. Figure $6 \mathrm{~b}$ shows 

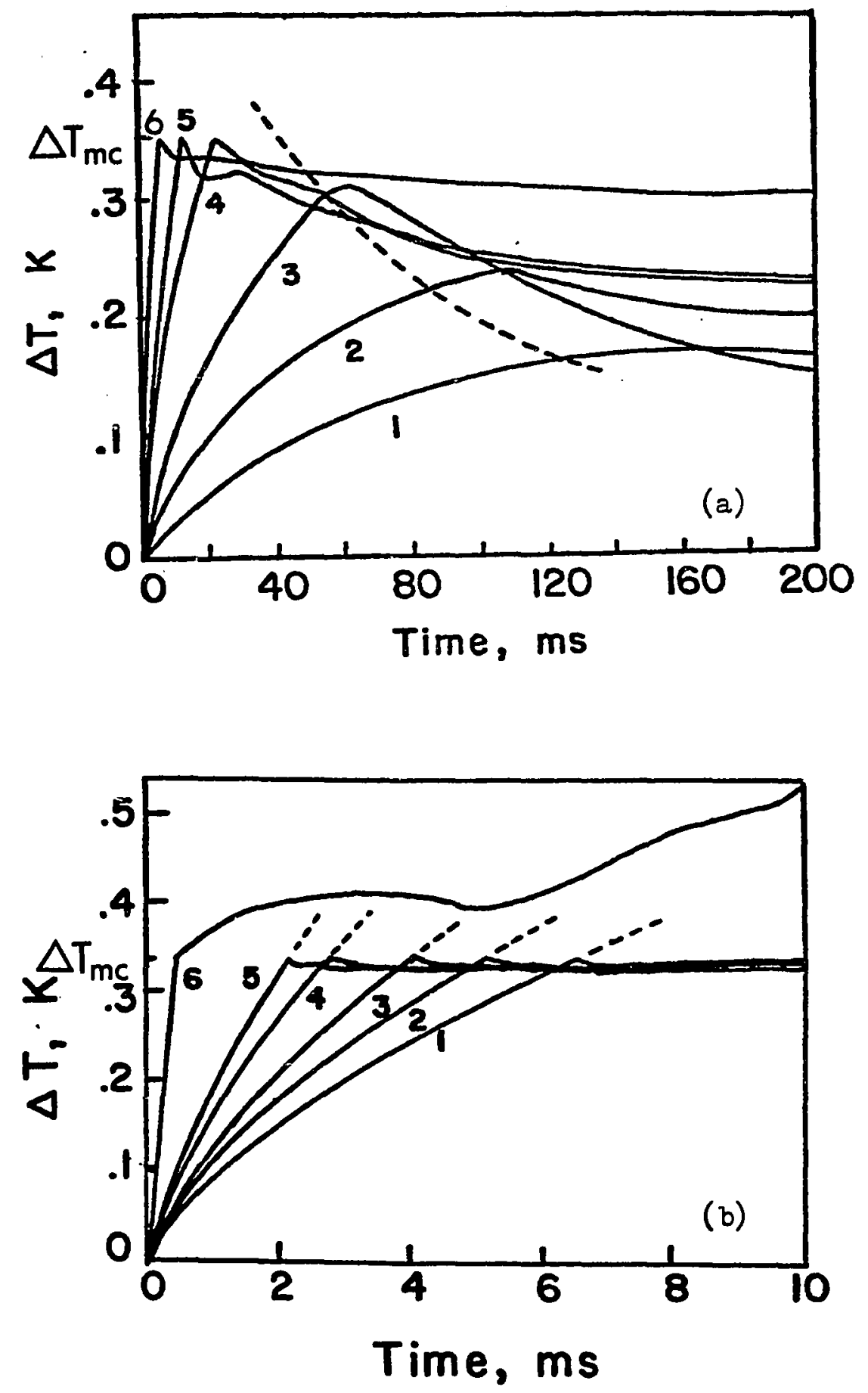

Figure 6. Superheat temperature $\Delta T$ vs. time. Transient superheat curves are shown for low and high range of applied powers. The bath temperature is $4.2 \mathrm{~K}$. The numbers labelling each curve represents the applied powers ( (a) 1) 5, 2) 9, 3) 15,4$)$, 29, 5) 41, 6) 72 and (b) 1) 58 , 2) 70, 3) 80,4$) 106$, 5) 127,6$) 430 \mathrm{mw} / \mathrm{cm}^{2}$. 
significantly higher power transient curves and much shorter time scales. (The significane of the dashed curves in Figs. $6 \mathrm{a}$ and $6 \mathrm{~b}$ are discussed in the next section.) The constancy of the maximum overshoot temperature is typically illustrated in Fig. 7, where $\Delta \mathrm{T}_{\mathrm{m}}$ is plotted as a function of the applied power for several values of the helium bath temperature $T_{b}$. For example, at $T_{b}=4.2 K, \Delta T_{m}$ is constant for applied powers greater than approximately $35 \mathrm{~mW} / \mathrm{cm}^{2}$ up to the maximum value used, roughly $420 \mathrm{~mW} / \mathrm{cm}^{2}$ (Fig. 7 only shows data up to a maximum of $120 \mathrm{~mW} / \mathrm{cm}$ ). At each bath temperature, the portion of the curves in Fig. 7 for which $\Delta \mathrm{T}_{\mathrm{m}}$ is constant corresponds to a sharp peak in the overshoots on a $\Delta T$ vs. time plot. Note also in Fig. 7 that $\Delta \mathrm{T}_{\mathrm{mc}}$ increases with decreasing bath temperature. Such characteristic behavior of the temperature response was not observed by Pavlov and Skripov (1965, 1967, 1970) in their pulse heating experiments.

At significantly higher powers, roughly above $400 \mathrm{mw} / \mathrm{cm}^{2}$, the sharp peak in the overshoot takes the shape of a kink and the temperature beyond that continually rises toward a steady film boiling condition. These kinks are found to be at the same superheat $\Delta T_{m c}$ as the sharp peaks at any given bath temperature (Fig. 6b). The lowest bath temperature at which the above behavior in the transient temperature response could be observed is the superfluid transition temperature. Although it is not shown in Fig. 7 , as the bath temperature is lowered through the $\lambda$ - point, 


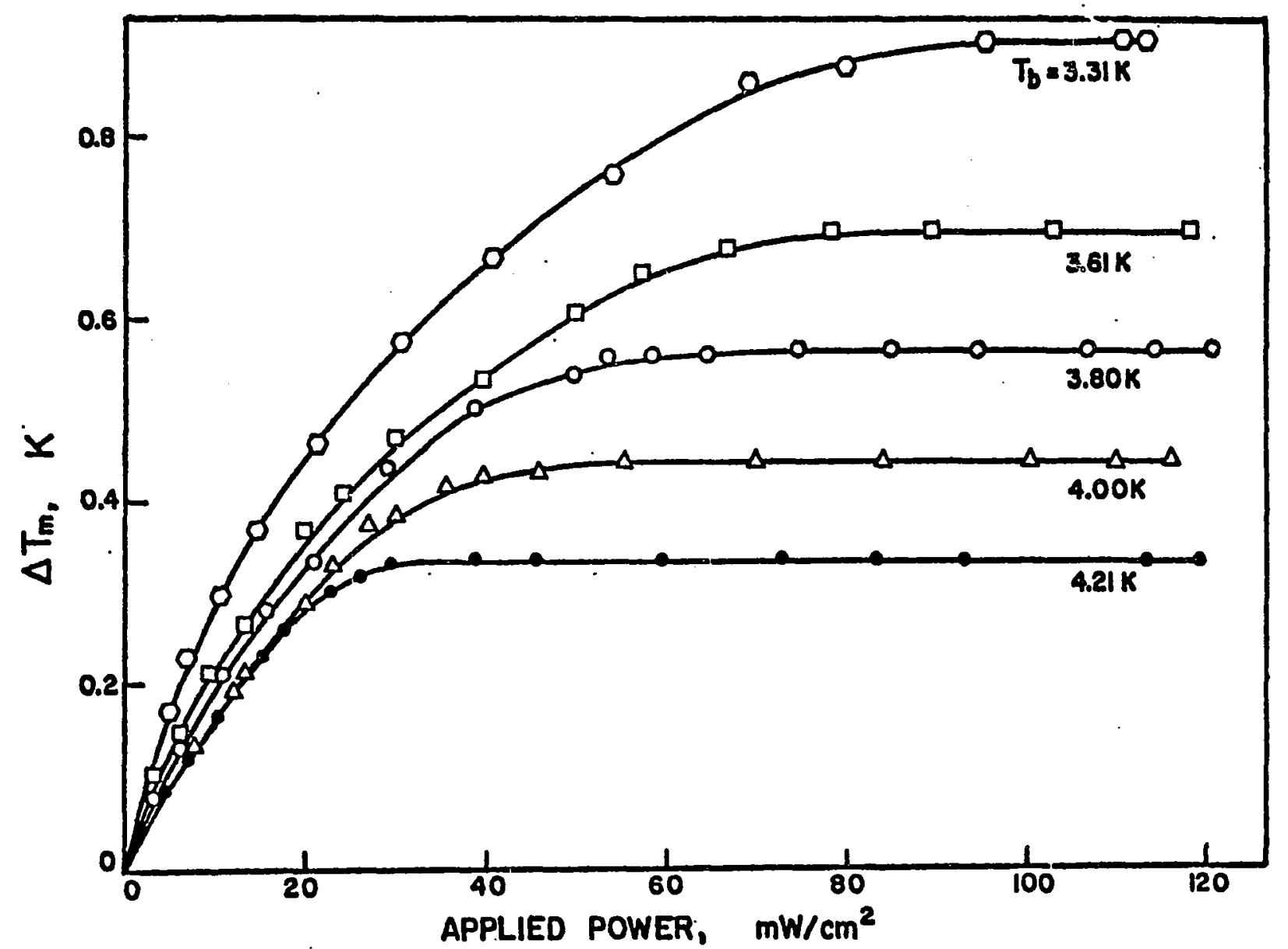

Figure 7. Maximum superheat temperature as a function of applied power. Maximum superheat temperatures $\Delta \mathrm{T}_{\mathrm{m}}$ are plotted as a function of applied power $\mathrm{P}$ for several bath temperatures. ${ }^{\mathrm{m}}$ For each bath temperature, $\Delta \mathrm{T}_{\mathrm{m}}$ approaches a constant value $\Delta \mathrm{T}_{\mathrm{mc}}$, as $\mathrm{P}$ increases. 
all indications of superheat and overshoot disappear. As a result, the lower temperature limit of this transient superheating technique is the superfluid transition temperature in the case of ${ }^{4}$ He. The upper bath temperature limit in our experiment was roughly $4.6 \mathrm{~K}$ because of the risk involved in pressurizing the glass Dewar beyond 1.25 atmospheres.

\section{DISCUSSION OF EXPERIMENTAL INTERPRETATIONS}

We suggest that the sharp peaks in the overshoots which occur at a constant superheat $\Delta \mathbf{T}_{\mathrm{mc}^{\prime}}$ result from the very rapid onset of homogeneous nucleation when the superheat temperature of a thin layer of liquid helium in contact with the heater surface reaches the homogeneous nucleation temperature. The temperature gradient in the superheated thermal boundary layer corresponding to applied powers above $30 \mathrm{~mW} / \mathrm{cm}^{2}$ is estimated (Lurie and Johnson 1962) to be of the order of $10 \mathrm{mK}$ per micron. On the other hand, the rounded overshoots occur due to a time delay in the onset of heterogeneous nucleation. In the initial stages, the liquid helium in contact with the heater surface is superheated primarily due to thermal conduction. At an appropriate superheat, depending on the heating rate and the surface condition of the heater, activation of nucleation sites takes place and heterogeneous nucleation then proceeds at a relatively gradual rate because the potential surface irregularities are randomly distributed in size, 
shape, and density. At higher applied powers, roughly above $30 \mathrm{~mW} / \mathrm{cm}^{2}$, the rate of heating is faster and the superheat of the liquid helium layer adjacent to the heater surface reaches the homogeneous temperature before appreciable heterogeneous nucleation at the interface can prevent it from doing so.

It may at first seem surprising that the homogeneous nucleation temperature can be attained by heating a solid in a liquid. As a result, it is worth stating in detail the reasons for our interpretation that the sharp temperature peaks are due to homogeneous nucleation. We cite the following eight arguments in support of this claim.

1. Due to the excellent wettability (the liquidsolid contact angle is near zerol and low surface tension of liquid helium, the presence of vapor-filled surface cavities is unlikely. Moreover, due to the low temperatures involved, there can be no dissolved impurity gases present inside the cavities. Consequently, in the initial stages of superheating there are no active nucleation sites on the solid surface to start with and the superheating of the liquid helium takes place solely due to thermal conduction from the heater into the liquid. This fact is confirmed by the excellent agreement between the observed superheat temperature in the initial stages of heating and predictions based on the assumption that heat is removed from the solid surface by thermal conduction into the liquid (Lurie and Johnson 1962). In Fig. 6a the dashed 
curve represents the locus of the points which mark the instances at which the temperature response is observed to begin deviating from the predicted temperature response based on the thermal conduction assumption. This situation occurs due to the finite delay time $\tau$ involved with the onset of heterogeneous nucleation. Consequently, if the superheating takes place on a time scale less than $\tau$, then the liquid can be superheated up to the homogeneous nucleation temperature by thermal conduction without significant competition from heterogeneous nucleation. Fig. $6 \mathrm{~b}$ shows the typical excellent agreement of the temperature response predicted from the conduction assumption (dashed lines) with the observed transient superheat temperature. This agreement supports our assumption that in time scales $t<\tau$, heterogeneous nucleation is not likely to be present in the superheating process.

2. The abrupt change in the characteristics of the temperature overshoots from rounded to sharp peaks strongly suggest that two different processes may be involved. The fact that the sharp peaks occur only at times less than $\tau$, the delay time for inception of heterogeneous nucleation, is an indication that the sharp peaks are caused by a sudden onset of homogeneous nucleation in a thin superheated layer of liquid helium. The sudden drop in temperature associated with the sharp peak is believed to be due to extraction of thermal energy from the heater surface for subsequent 
growth of the critical nuclei formed in the process of homogeneous nucleation.

3. The sharp peaks in the temperature overshoots occur at a constant superheat temperature $\Delta \mathrm{T}_{\mathrm{mc}}$ ' independent of applied power. In our first argument we show evidence that up to the development of the sharp peaks, heterogeneous nucleation is not involved. The characteristic constant superheat associated with the sharp peaks further supports our argument because it is highly unlikely that a random process such as heterogeneous nucleation could produce a temperature pattern with such reproducibility and also be independent of the applied power. On the other hand, the probability of homogeneous nucleation becomes significant in practice only above a very narrowly defined superheat temperature range and the resulting nucleation process is extremely rapid. It is worth mentioning at this point that the exact value $\Delta \mathrm{T}_{\mathrm{mc}}$ has been reproduced at any given bath temperature with different bismuth crystals of varied surface conditions, using both an electropolished surface and surfaces filled with etch pits. The lack of influence of the surface microgeometry on the superheat $\Delta T_{m c}$ is further evidence that the sharp peaks are not due to a sudden onset of heterogeneous nucleation. In contrast, the homogeneous nucleation temperature is a characteristic property of the liquid only and does not depend on the microgeometry of the solid surface. It is also unlikely that such repeatable constant superheat temperatures can 
result from an adsorption-desorption process as suggested by Purdy et al. (1972) in connection with their study of steady-state heat transfer in liquid helium.

4. We found that once nucleation takes place on the surface, it takes several seconds before the nucleation sites are deactivated, i.e. become filled with liquid helium. If the heating current is turned back on before that time, the effects of preexisting active nucleation sites can be clearly observed. Such effects manifest themselves through the lowering of the peak superheat temperatures of the rounded overshoots at any given applied power. The effects can also be observed in the case of those sharp peaks which are associated with relatively lower power values (around 30-40 $\mathrm{mW} / \mathrm{cm}^{2}$ at atmospheric pressure). Fast repeated pulsing tends to make these sharp peaks rounded. However, at higher power values, the influence of the preexisting active nucleation sites are of diminishing importance. This can be inferred from the fact that the higher power sharp peaks are not affected and the original behavior of constant superheat $\Delta \mathrm{T}_{\mathrm{mc}}$ associated with such sharp peaks can still be observed. The only difference in the case of preexisting active nucleation sites is that the $\Delta T_{m}$ vs. $P$ characteristics shown in Fig. 7 are shifted slightly toward higher powers. This result is consistent with that of the pulse heating method of Pavlov and Skripov (1965, 1967, 1970) used in organic liquids. They also find that at high heating rates, 
attainment of homogeneous nucleation temperature is possible even in the presence of active nucleation sites.

5. The experimentally determined superheat values, $\Delta \mathbf{T}_{\text {mc }^{\prime}}$ at wide range of bath temperatures, $\mathbf{T}_{b^{\prime}}$ are found to be in excellent agreement with the estimates based on the predictions from the homogeneous nucleation theory. In Table I we present the experimental data on the homogeneous nucleation temperature, $T_{h}=T_{b}+\Delta T_{m c}$, and the theoretical values derived from Eq. (3.4) for a nucleation rate of $\mathrm{J}$ equal to one critical nuclei/ $\mathrm{cm}^{3} / \mathrm{sec}$. This value of $J$ was selected somewhat arbitrarily, based on the heating rate employed in this study. Due to the extreme insensitivity of the homogeneous nucleation temperature to the nucleation rate, the error involved is insignificant. For example, some twelve orders of magnitude increase in the J value corresponds to a mere one per cent increase in the superheat of liquid helium at atmospheric pressure. Figure 8 illustrates the dependence of the homogeneous nucleation temperature on the nucleation rate. It should be mentioned that in deriving the kinetic coefficient in the preexponential factor on Eq. (3.4), viscous and inertial forces which affect the dynamics of the growth of bubbles as well as the thermal regime of growth were not taken into account. The effect of these factors has been considered by Kagan (1960), and are negligible in the case of liquid helium. The estimated values of the parameters $\delta_{\lambda}$, $\omega$ and $\omega^{\prime}$ for liquid helium, which characterize 


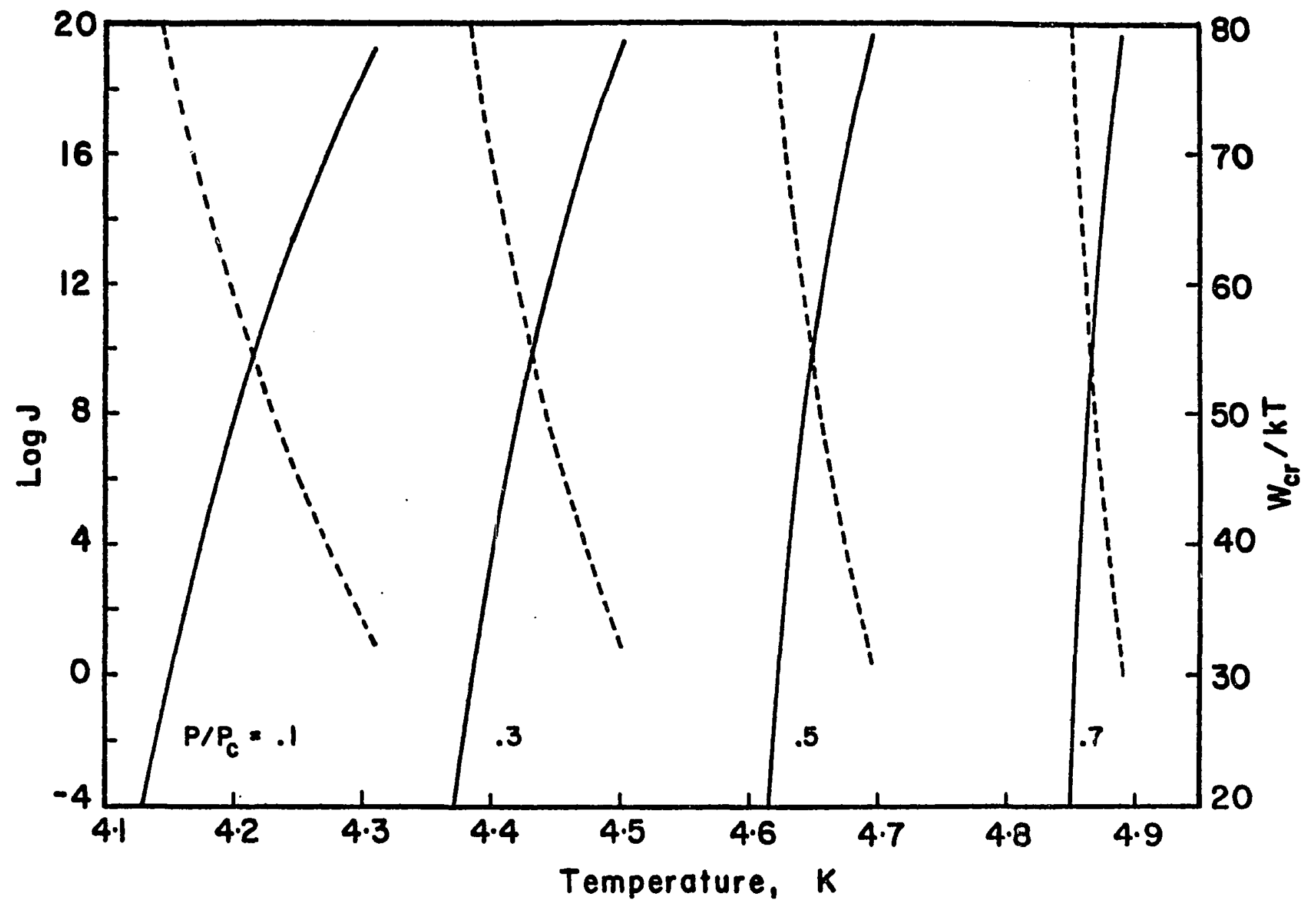

Figure 8. Temperature dependence of $J$ and $W_{0}$. Temperature dependence of the nucleation rate and the work of formation of the critical nucleus (dashed line) in superheated liquid helium are shown for four different bath pressures. $P_{c}$ is the critical pressure of helium. 
the hydrodynamical constraints (see Kagan 1960 and Blander 1979 for details), are of the order of $2.5 \times 10^{-4}, 10.5$ and 55, respectively.

TABLE I

HOMOGENEOUS NUCLEATION TEMPERATURE $\left(T_{h}\right)$ OF LIQUID HELIUM

\begin{tabular}{|c|c|c|}
\hline \multirow{2}{*}{$\begin{array}{c}\text { Bath Temp. } \\
\text { (K) }\end{array}$} & \multicolumn{2}{|c|}{ Homog. nucl. temp. (Th) } \\
\cline { 2 - 3 } & Exp. (K) & Theo. (K) \\
\hline 4.616 & 4.763 & \\
4.588 & 4.750 & 4.766 \\
4.559 & 4.733 & 4.750 \\
4.529 & 4.710 & 4.733 \\
4.468 & 4.683 & 4.715 \\
4.499 & 4.699 & 4.681 \\
4.437 & 4.677 & 4.698 \\
4.406 & 4.648 & 4.664 \\
4.373 & 4.633 & 4.647 \\
4.340 & 4.622 & 4.629 \\
4.307 & 4.593 & 4.613 \\
4.272 & 4.585 & 4.595 \\
4.210 & 4.550 & 4.578 \\
3.993 & 4.453 & 4.555 \\
3.907 & 4.407 & 4.450 \\
3.742 & 4.355 & 4.415 \\
3.672 & 4.322 & 4.353 \\
3.548 & 4.289 & 4.328 \\
3.497 & 4.275 & 4.288 \\
3.247 & 4.212 & 4.271 \\
2.992 & 4.152 & 4.210 \\
2.792 & 4.120 & 4.142 \\
2.587 & 4.052 & 4.120 \\
& & 4.097 \\
\hline & & \\
\hline & &
\end{tabular}

The surface tension values used in estimating $T_{h}$ are based upon an interpolation of the measurements of Allen and Misener (1938). More recent measurements of the surface tension of helium I by Dickson et al. (1970) and King and Wyatt (1971) are in excellent agreement with the earlier 
measurements of Allen and Misener (1938) . Other thermophysical properties are taken from Mccarty (1973). The overall estimated uncertainty in the experimental measurement of $\mathbf{T}_{\mathrm{h}}$ is $\pm 10 \mathrm{mk}$. The error in the bath temperature is $\pm 2 \mathrm{mK}$ at higher temperatures and $\pm 6 \mathrm{mk}$ at the lower bath temperatures.

6. Similar transient superheating experiments performed by us using platinum wire in liquid nitrogen (Chapter IV) and oxygen are consistent with the observations in liquid helium. Excellent agreement with theory has been observed in all cases over a wide range of reduced bath pressures. Moreover, the inception of nucleation in the case of liquid nitrogen was studied using laser scattering and acoustic transducers. The results obtained from these studies correspond in many respects with the liquid helium studies. We found strong correlation between inception of heterogeneous nucleation and deviation of the observed transient superheat response from the predicted response based on a conduction assumption. In addition, the laser scattering experiments in liquid nitrogen are consistent with the sudden onset of nucleation at the sharp temperature overshoots. The results of the transient superheating experiments in liquid nitrogen provide us with an independent corroboration of the experimental technique.

7. That the observed constant superheat $\Delta T_{\text {mc }}$ does in fact represent the homogeneous nucleation superheat temperature which is not affected by background radiation 
or cosmic rays can be inferred from the lack of random fluctuations in $\Delta \mathbf{T}_{\mathrm{mc}} \cdot$ To further validate this assumption, we subjected the heater surface to $\mathrm{x}$-rays from a radioactive source placed in the close proximity of the heater surface. The same value of superheat $\Delta \mathbf{T}_{\mathrm{mc}}$, at any given bath temperature, could be reproduced exactly, but at slightly higher heating rates than normal.

8. In our thermometry technique we have used an external magnetic field. Questions may be raised regarding a possible effect of this magnetic field on the measured superheat temperature $\Delta \mathrm{T}_{\mathrm{mc}} \cdot$ Since liquid helium is slightly diamagnetic compared to helium vapor, there is a possibility that an applied magnetic field may influence the measured homogeneous nucleation temperature of liquid helium. This effect is believed to be due to the difference in the magnetostatic energy in the liquid and the vapor nuclei. This energy difference causes a reduction in the pressure inside critical vapor nuclei which then acts to increase the height of the thermodynamic barrier slightly and consequently raises the homogeneous nucleation temperature. The estimated effect on the temperature turns out to be less than $1 \mu \mathrm{K}$. An experimental check on this was made by repeating the transient superheating experiments at different magnetic fields. Changing the magnetic field from $0.4 \mathrm{~T}$ to $1.5 \mathrm{~T}$ did not produce any detectable change in the observed maximum superheat $\Delta \mathrm{T}_{\mathrm{mc}} \cdot$ Therefore, we assumed that the effect of the magnetic field is insignificant. 
In summing up the arguments presented above, we first showed that the process of superheating up to the sharp peaks is primarily due to thermal conduction; heterogeneous nucleation is not involved. Next, we argued that the probability of nucleation in liquid helium due to homogeneous nucleation is greater than that of heterogeneous nucleation. We then present evidence that the sharp peaks must be due to a sudden onset of homogeneous nucleation in the superheated liquid layer in contact with the heater surface. Finally, we show that the measured homogeneous nucleation temperatures are not affected either by cosmic rays or by the external magnetic field used in thermometry. It is pertinent at this point to comment on the appropriateness of using the homogeneous nucleation equation (3.4) which gives us a steady-state nucleation rate, in deriving theoretical estimates for comparison with a transient experiment. For nonsteady-state conditions, Eq. (3.4) changes to (Abraham 1974)

$$
J(t)=J\left[1-\exp \left(-t / \tau_{s}\right)\right]
$$

where $J$ is the steady-state nucleation rate and $\tau_{s}$ is the characteristic relaxation time for the establishment of steady-state nucleation. An estimate of this relaxation time for superheated liquid helium over a wide range of bath temperatures shows $\tau_{s}$ to be extremely short, varying roughly from 2 to $10 \mathrm{~ns}$. Since this relaxation time is 
several orders of magnitude smaller than the time scale of our superheating experiment, 0.1 to $30 \mathrm{~ms}$, the use of the steady-state expression (3.4) is quite justified.

\section{RESULTS}

The experimental superheat data $\Delta \mathbf{T}_{\mathrm{mc}^{\prime}}$ corresponding to homogeneous nucleation in Iiquid helium can be satisfactorily expressed in terms of the scaled temperature $\mathbf{T} / \mathbf{T}_{\mathbf{C}}$ by

$$
\Delta \mathrm{T}_{\mathrm{mc}}=\Delta \mathrm{T}_{\text {ref }}\left(1-\frac{\mathrm{T}}{\mathrm{T}_{\mathrm{C}}}\right)^{1.529} ;\left(\Delta \mathrm{T}_{\text {ref }}=4.268 \mathrm{~K}\right)
$$

The theoretical estimates for the homogeneous nucleation temperature $T_{h^{\prime}}$ corresponding to a nucleation rate of $\mathrm{J}=1$, can be expressed in terms of the pressure as

$$
T_{h}=T_{c}\left[1-.2238\left(1-\frac{P}{P_{C}}\right)^{1.0001}\right]
$$

where $P_{C}$ and $T_{C}$ are the critical pressure and critical temperature of liquid helium respectively, and $\mathrm{P}$ is the ambient bath pressure. Homogeneous nucleation temperature for nucleation rates ranging from $J=10^{-4}$ to $10^{20}$ nuclei/cm $3 / \mathrm{sec}$ at several reduced pressures can be obtained from Fig. 8 .

A simplified form of the nucleation rate equation (3.4) which is valid over the entire range of bath pressures studied, can be expressed as 


$$
\text { ln } J=76.3-G
$$

where, $J=$ number of critical nuclei $/ \mathrm{cm}^{3} / \mathrm{sec}$. $G=W_{C r} / k T$ is the Gibb's number and $w_{c r}=$ work of formation of a critical nuclei.

The Gibb's number contains the most essential information on the stability of the metastable liquid and frequency of spontaneous nucleation. The significance of the Gibb's number is that it represents the ratio of the height of the activation barrier of the free energy in homogeneous nucleation to the mean energy of thermal motion per degree of freedom of the system. The magnitude $k T$ is the natural measure of the thermodynamic fluctuations to which the metastable phase is subjected. This magnitude also shows how the Gibb's number $G$ characterizes the stability of the metastable phase. The dashed lines in Fig. 8 represent the behavior of the Gibb's number as a function of temperature at several reduced pressures.

Referring back to the three arguments we made earlier, in connection with the explanation of the lack of agreement of tensile strength experiments with the predictions from the homogeneous nucleation theory, we have shown that none of the arguments can be supported on the basis of our experimental data in superheated liquid helium. We find that the homogeneous nucleation theory does describe the nucleation behavior of superheated liquid helium quite 
well. The excellent agreement of our experimental nucleation data with theory confirms the validity of the theory for a quantum fluid such as liquid helium. No obvious modification of the existing nucleation theory due to any quantum effects seem necessary. Moreover, no different mechanism of nucleation needs to be invoked. As it has been pointed out in the theory section, nucleation due to quantum tunneling of the thermodynamic barrier may be of significance only at very low temperatures $-0.3 \mathrm{~K}$ (Lifshitz and Kagan 1972). One is likely to observe the tunneling nucleation, if at all, only in tensile strength experiments and not in superheating experiments as in this work.

In the homogeneous nucleation temperature calculations the surface tension $\gamma$ used was for a plane interface. Equation (3.4) is extremely sensitive to the accuracy of the surface tension data employed. Therefore, the excellent agreement between the theoretical and experimental homogeneous nucleation temperatures is suggestive of the fact that the interface curvature effects might be negligible for the investigated superheating in liquid helium (the liquid-vapor interface in the case of liquid helium should be considered as relatively diffused due to the large thermal de Broglie wavelength $5 \AA$ associated with the helium atoms). On the basis of such agreement between theory and experiment, it can be estimated that down to about $30 \AA$ radius of the critical vapor nuclei in 
liquid helium the curvature effects, if there are any, are not detectable. This result is consistent with investigations in other superheated liquids (Skripov et al. 1979, Skripov and Sinitsyn 1966). However, such a situation may not be valid in the case of strongly expanded liquids $\left(P_{I}<0\right)$ as encountered in tensile strength experiments. The vapor density at the temperatures at which such expanded states are possible (e.g. in He II) is very low. Consequently, the critical bubble is practically empty, while in the superheating case $\mathrm{P}_{\mathrm{I}}>0\left(\mathrm{~T}_{\mathrm{h}} / \mathrm{T}_{\mathrm{C}} \sim .8\right.$ to 1$)$, we have a relatively large number of vapor molecules in the critical nuclei, $n_{c r}=10^{2}-10^{3}$. Moreover, the critical nucleus associated with tensile strength experiments ( $10 \AA)$ is considerably smaller than that encountered in superheated Iiquid helium, 30 - $80 \AA$. These factors may contribute to a modification of the nucleation theory, particularly the capillarilty approximation, as applicable to the case of tensile strength experiments in He II. For instance, the ripplon contribution (Atkins and Narhara 1965) to the bulk $\gamma$ may need to be replaced by that due to quantized surface deformations of the critical bubble (Marston 1976). 
CORRESPONDING STATES ANALYSIS

Phenomenological analyses based on the principle of corresponding states have shown that this provides a useful method for systematizing the measured properties of one or more substances to predict the properties of other substances under conditions for which no data exist. The virtue of the corresponding states method consists in its simplicity and its universality. This principle was originally formulated by van der wals for classical systems. Subsequently, a method of accounting for deviations caused by quantum effects was suggested by Byk $(1921,1922)$. The further development of the quantum mechanical principle of corresponding states has been primarily due to de Boer and his co-workers (1948a, 1948b, 1948c).

In the classical empirical system of corresponding states, the units which are used to reduce the state variables are the critical point values $P_{C}, V_{C}$, and $T_{C}$. There is, however, nothing unique about this choice of units. For instance, an alternative formulation of the law of corresponding states is due to de Boer and his collaborators. In this formulation, which is a deduction from the statistical mechanical expressions for the partition function and configurational integral, the intermolecular field parameters are used to reduce the state variables. 
A good analytical approximation of the intermolecular interaction is given by the Lennard-Jones spherically symmetrical 6-12 potential (1924). This potential may be written

$$
u(r)=4 \varepsilon\left[(\sigma / x)^{12}-(\sigma / x)^{6}\right]
$$

where $\varepsilon$ is the depth of the potential field and $\sigma$ is the value of $r$ for which $u(r)=0$. The two parameters of the Lennard-Jones potential provide a convenient scaling to express the thermodynamic variables of interest as reduced (dimensionless) quantities, as follows:

$$
\begin{gathered}
\mathrm{T}^{*}=\mathrm{k} \frac{\mathrm{T}}{\varepsilon}, \quad \mathrm{P}^{*}=\frac{\sigma^{3}}{\varepsilon} \mathrm{P}, \quad \gamma^{*}=\frac{\sigma^{2}}{\varepsilon} \gamma \\
\mathrm{N}^{*}=\mathrm{N} \sigma^{3}, \quad \mathrm{t}^{*}=\left(\frac{\varepsilon}{\mathrm{m} \sigma^{2}}\right)^{\frac{1}{2}} \mathrm{t}
\end{gathered}
$$

where $\mathrm{k}$ is the Boltzman constant, $\mathrm{m}$ the molecular mass, $N$ the number density, $t$ the time, $\gamma$ the surface tension, $\mathbf{P}$ the pressure, and $\mathbf{T}$ the temperature. With the above reduced quantities the homogeneous nucleation equation, Eq. (3.4), can also be expressed in dimensionless form as

$$
J^{*}=N^{*}\left(\frac{2 \gamma^{*}}{\pi B}\right)^{\frac{1}{2}} \exp \left[\frac{-16 \pi \gamma^{* 3}}{3 T^{*}\left(P_{V}^{*}-P_{L}^{*}\right)^{2}}\right]
$$

where $J^{*}\left(J^{*}=\sigma^{4} V(m / \varepsilon)\right)$ is the number of critical nuclei 
per unit reduced time per unit reduced volume.

The advantage of the intermolecular unit formulation over the older empirical formulation is that it enables one to investigate the deviations from classical thermal behavior caused by quantum effects, which occur with the lighter molecules whose fluid ranges extend to lower temperatures. The dimensionless parameter $\Lambda=\mathrm{h} /(\sigma \sqrt{\mathrm{m} \varepsilon})$ as defined by de Boer, is referred to as the quantum parameter for the law of corresponding states. It is the ratio of the de Broglie wavelength, $h / \sqrt{ } \mathrm{m} \varepsilon$, of a molecule whose mass is $\mathrm{m}$ and kinetic energy $\varepsilon / 2$, to the average distance between the molecules as characterized by the parameter $\sigma$ of the Lennard-Jones potential. Table II presents the value of the de Boer quantum parameter, and the LennardJones parameters for the liquids employed in this study. These values are taken from Rogers and Brickwedde (1966). We now show the applicability of the quantum mechanical principle of corresponding states to the case of homogeneous nucleation of the liquid noble gases such as $\mathrm{Ar}, \mathrm{Kr},{ }^{4} \mathrm{He}$, and ${ }^{3} \mathrm{He}$. We have also included $\mathrm{H}_{2}$ since its interatomic potential can be assumed to be approximately spherically symmetric (Rogers and Brickwedde 1966). This is due to the fact that the population of excited rotational states of para- $_{2}$ below the critical temperature is low, inelastic collisions are essentially nonexistent and a spherically symmetric force field is applicable. 
TABLE II

THE MOLECULAR PARAMETERS FOR 6-12

LENNARD-JONES FLUIDS

\begin{tabular}{lccc}
\hline Fluid & $\Lambda$ & $\varepsilon / \mathrm{k}(\mathrm{K})$ & $\sigma(\AA)$ \\
\hline $4_{\mathrm{He}}$ & 2.678 & 10.22 & 2.556 \\
$3_{\mathrm{He}}$ & 3.085 & 10.22 & 2.556 \\
$\mathrm{Ar}$ & .186 & 119.8 & 3.405 \\
$\mathrm{Kr}$ & .103 & 165.2 & 3.66 \\
$\mathrm{Xe}$ & .063 & 230 & 3.99 \\
$\mathrm{H}$ & 1.720 & 36.7 & 2.959 \\
$\mathrm{HD}$ & 1.542 & 35.94 & 2.955 \\
$\mathrm{D}_{2}$ & 1.245 & 35.2 & 2.952 \\
$\mathrm{~T}_{2}$ & 1.029 & 34.5 & 2.949 \\
\hline
\end{tabular}

The value of the de Boer parameter $\Lambda$ for HD has been computed after Friedman (1962), see Eq. (3.19).

In Fig. 9 we present the homogeneous nucleation temperature $\mathrm{T}_{\mathrm{h}}$ data for $\mathrm{Ar}, \mathrm{Kr}, \mathrm{Xe},{ }^{4} \mathrm{He}$, and $\mathrm{H}_{2}$ in terms of their scaled temperatures $T_{h} / T_{C}$ and scaled bath pressures $P / P_{C}$. The experimental data for $\mathrm{Ar}, \mathrm{Kr}$, and $\mathrm{Xe}$ are taken from recent capillary boiling experiments of Skripov et al. (1979). The $\mathrm{H}_{2}$ data shown in Fig. 9 are selected bubble chamber data from Hord et al. (1964). The ${ }^{4}$ He data are from our transient superheating experiments discussed in this chapter. The solid lines are the theoretical predictions for each liquid from the homogeneous nucleation theory obtained by using Eq. (3.4). The dashed line represents the van der Waals spinodal shown only as a qualitative reference line. Note that all 


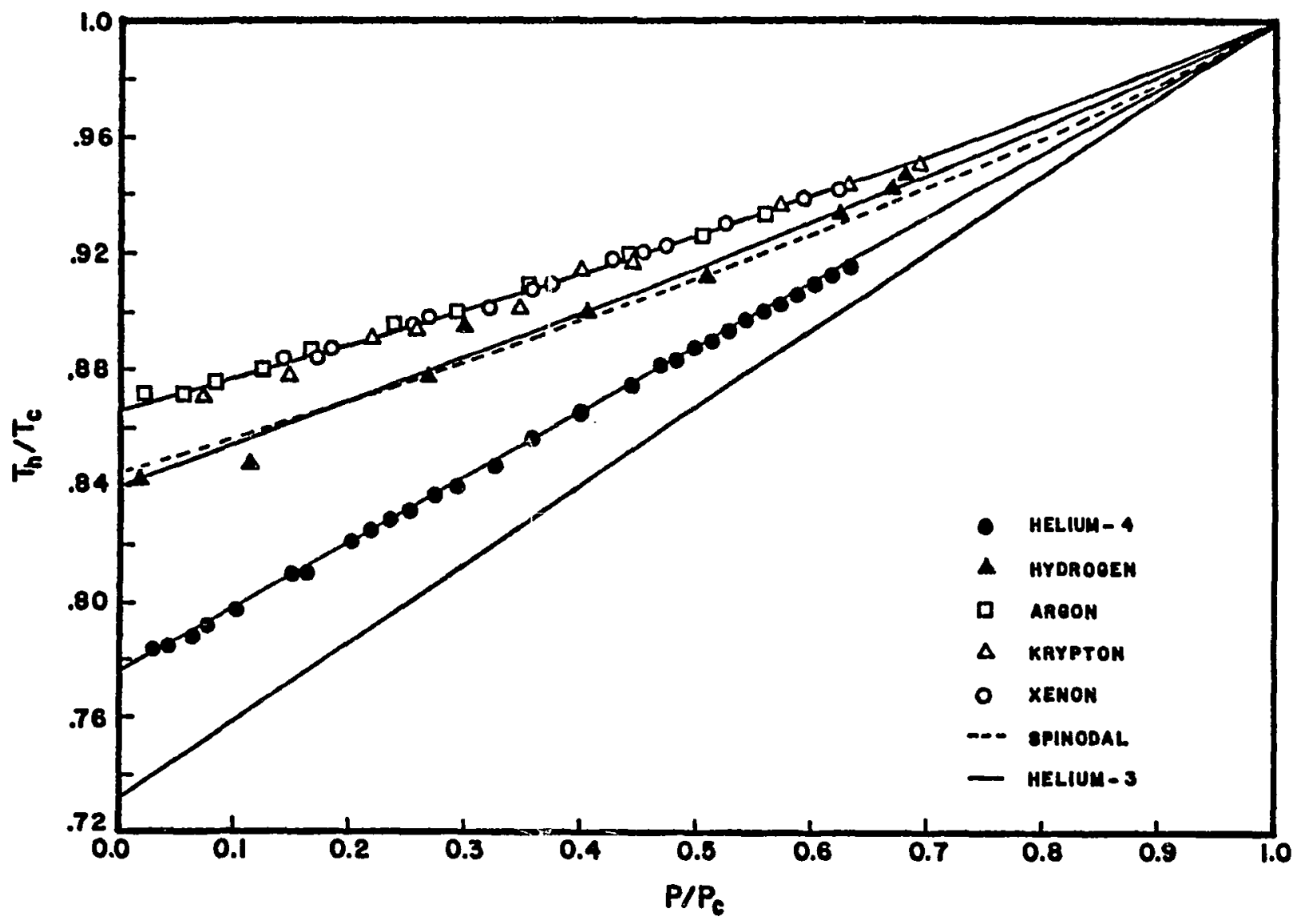

Figure 9. Scaled limits of superheat, $T_{h} / T_{c}$ versus scaled pressure, $P / P_{c}$. The homogeneous nucleation temperature $T_{h} / T h_{\text {is }}{ }^{c}$ plotted as a function of the bath pressure for $\mathrm{Ar}, \mathrm{Kr}, \mathrm{Xe}, \mathrm{H}_{2}$, and $\mathrm{He}$, where $\mathrm{T}_{\mathrm{c}}$ and $\mathrm{P}_{\mathrm{c}}$ are the critical temperature and pressure, respectively. The solid lines represent the predictions from Eq. $(3.4)$ for each liquid. The van der Waals spinodal is shown by the dashed line. 
the experimental $T_{h}$ data for the classical liquids such as $\mathrm{Ar}, \mathrm{Kr}$, and $\mathrm{Xe}$ fall on the same line clearly demonstrating the validity of the principle of corresponding states. The deviations of the experimental data and the theoretical predictions for the quantum liquids $\mathrm{H}_{2}$ and ${ }^{4} \mathrm{He}$, are obviously due to the quantum effects. At the present time there are no experimental data available for ${ }^{3}$ He. Therefore, only the theoretical predictions are shown. The magnitude of the deviations from the classical behavior is clearly proportional to the quantum parameter $\Lambda$.

The quantum effects on the homogeneous nucleation temperature are better illustrated in Fig. 10 in which the temperature and pressure are reduced using the LennardJones parameters. The significance of the dashed lines will be discussed later. The reduced homogeneous nucleation equation (3.13) describes the behavior of the three classical noble liquids $\mathrm{Ar}, \mathrm{Kr}$, and $\mathrm{Xe}$ as shown in Fig. 10.

We find that the available nucleation data for both the classical and quantum liquids can be quite satisfactorily correlated by an expression of the form

$$
\left(1-\frac{T_{h}}{T_{c}}\right)=m(\Lambda)\left(1-\frac{P}{P_{c}}\right)^{n(\Lambda)}
$$

which provides an explicit relationship between the homogeneous nucleation temperature and the saturation bath pressure. The parameters $m$ and $n$ are assumed to be dependent on the quantum parameter $\Lambda$ and their parametric 


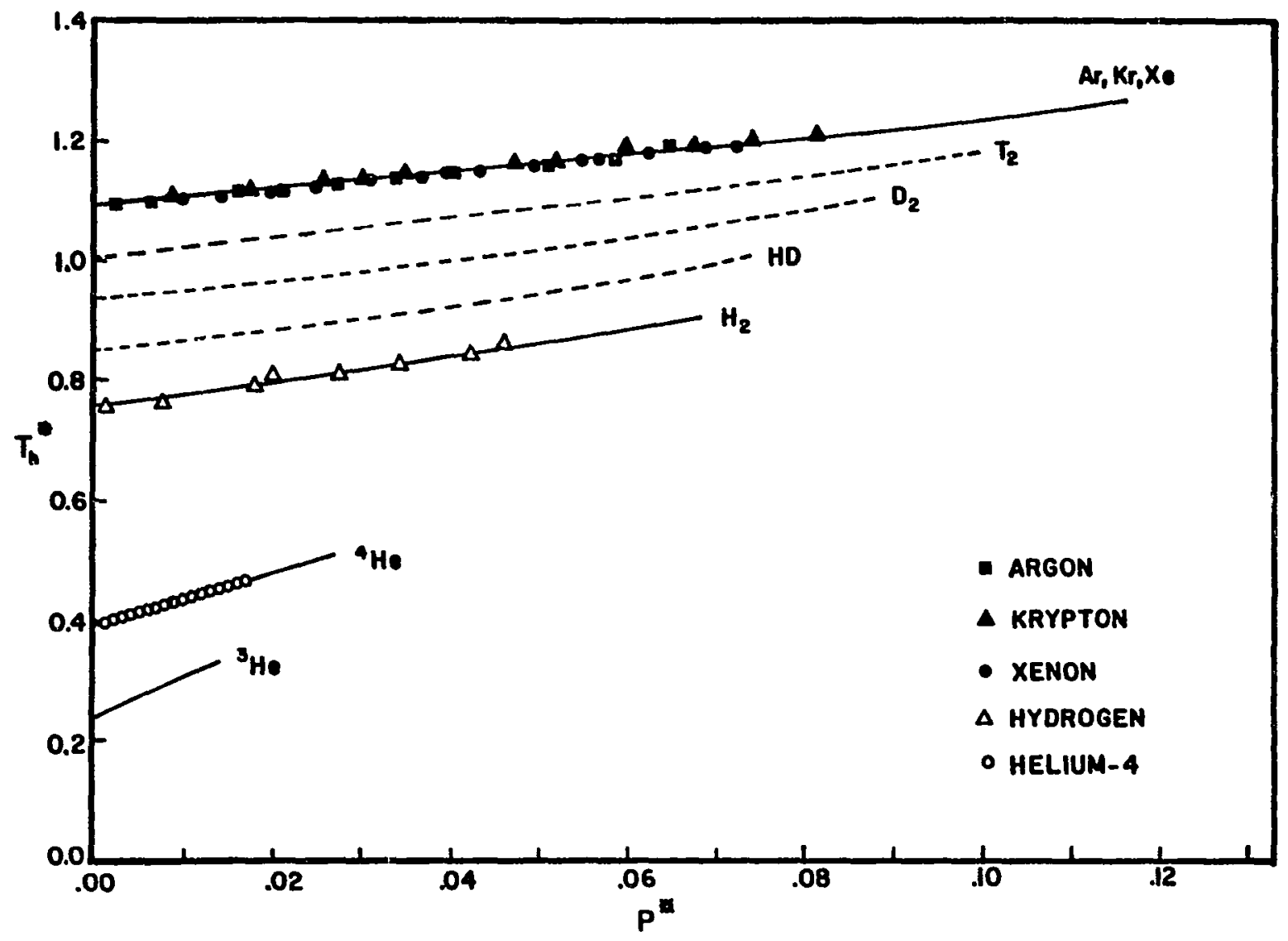

Figure 10. Reduced homogeneous nucleation temperature $\mathrm{T}^{*}$ vs. reduced pressure $\mathrm{P}^{*}$. The data shown in Fig. 9 are reduced according to $(3.12)$ and are plotted here. The dashed lines are the predictions of the homogeneous nucleation temperatures for the hydrogen isotopes $T_{2}, D_{2}$, and $\mathrm{HD}$. 
dependence on $\Lambda$ is illustrated in Fig. 11. The values of $\mathrm{m}$ and $\mathrm{n}$ for all the liquids considered were determined from a nonlinear least-squares curve fit of the predicted homogeneous nucleation temperature values obtained from Eq. (3.4) for a nucleation rate of $J=1$. The resulting slight deviations of the experimental data for $\mathrm{Ar}, \mathrm{Kr}$, and $\mathrm{Xe}$ is not serious. Since the nucleation data for different liquids are obtained from different experimental techniques the associated nucleation rates are different. We have chosen to standardize our correlation by selecting a reference value of $\mathrm{J}=1$ and using the theoretical values instead of the experimental data. The error in doing so is very small due to the insensitivity of the homogeneous nucleation temperature to the nucleation rate $\mathrm{J}$.

The parameters $m$ and $n$ can be expressed as a series in powers of $\Lambda^{2}$ as

$$
\begin{aligned}
m(\Lambda)= & .1403707+1.823961 \times 10^{-3} \Lambda^{2}+1.845064 \times 10^{-3} \Lambda^{4} \\
& -6.642568 \times 10^{-5} \Lambda^{6} \\
n(\Lambda)= & .9218464-1.999041 \times 10^{-4} \Lambda^{2}+2.916486 \times 10^{-3} \Lambda^{4} \\
& -1.906154 \times 10^{-4} \Lambda^{6}
\end{aligned}
$$

The coefficients in Eqs. (3.15) and (3.16) were determined from the values of $\mathrm{m}$ and $\mathrm{n}$ for $\mathrm{Ar}, \mathrm{H}_{2},{ }^{4} \mathrm{He}$ and ${ }^{3} \mathrm{He}$. As mentioned earlier, in reference to Fig. 10, the deviations of the $\mathrm{T}^{*}-\mathrm{P}^{*}$ curves corresponding to the 

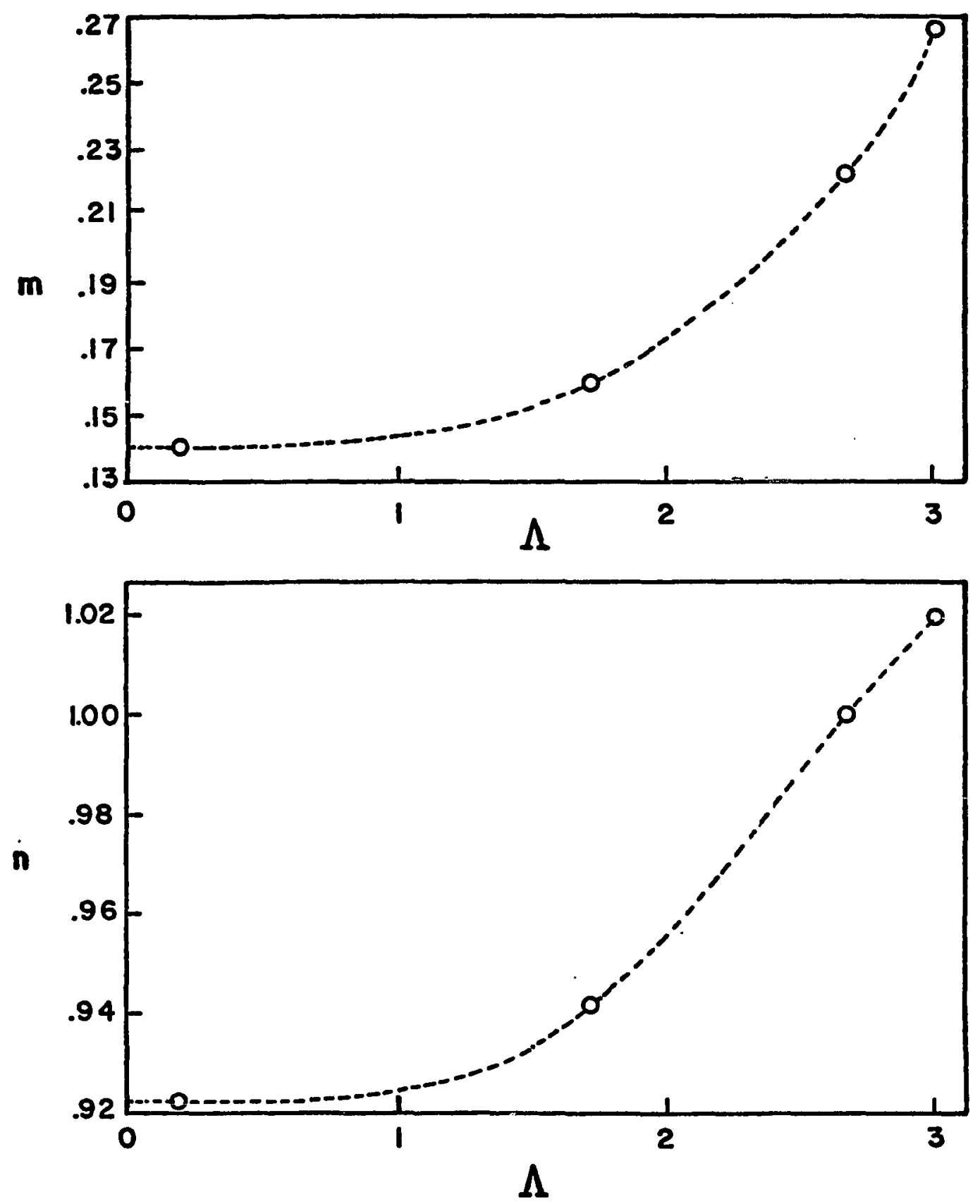

Figure 11. A plot of equations (3.15) and (3.16). $\Lambda$ is the de Boer quanfum parameter. The circles represent the values for $\mathrm{Ar}, \mathrm{H}_{2}$, $\mathrm{He}$, and ${ }^{3 e}$. 
quantum liquids from the single curve representing the classical fluid, are due to the fact that the quantum effects are not included. If the quantum parameter $\Lambda$ is incorporated as a scaling unit in addition to the two Lennard-Jones parameters $\varepsilon$ and $\sigma$ in reducing the variables then a single curve should represent the behavior of both the classical and the quantum liquids. A simple and straightforward way to accomplish this would be to define two variables containing $\Lambda$ as

$$
\begin{aligned}
& \Gamma=\left(1-\mathrm{T}_{h} / \mathrm{T}_{\mathrm{c}}\right) / \mathrm{m}(\Lambda) \\
& \text { and } \Pi=\left(1-\mathrm{P} / \mathrm{P}_{\mathrm{c}}\right)^{\mathrm{n}(\Lambda)}
\end{aligned}
$$

Figure 12 illustrates the nature of the quantum law of corresponding states as applied to the case of the homogeneous nucleation temperature. As expected, we find that a single line in the $\Gamma-\Pi$ plane satisfactorily represents the nucleation data for all the liquids, including the quantum liquids. The slight deviation of the data for Ar, $\mathrm{Kr}$, and $\mathrm{Xe}$ is due to the difference in nucleation rate as mentioned earlier.

The obvious advantage of such a quantum mechanical law of corresponding states is that on the basis of this one can now conveniently predict the homogeneous nucleation temperature of several hydrogen isotopes. A knowledge of the reduced pressure and the quantum parameter $\Lambda$ is sufficient to predict the reduced homogeneous nucleation 


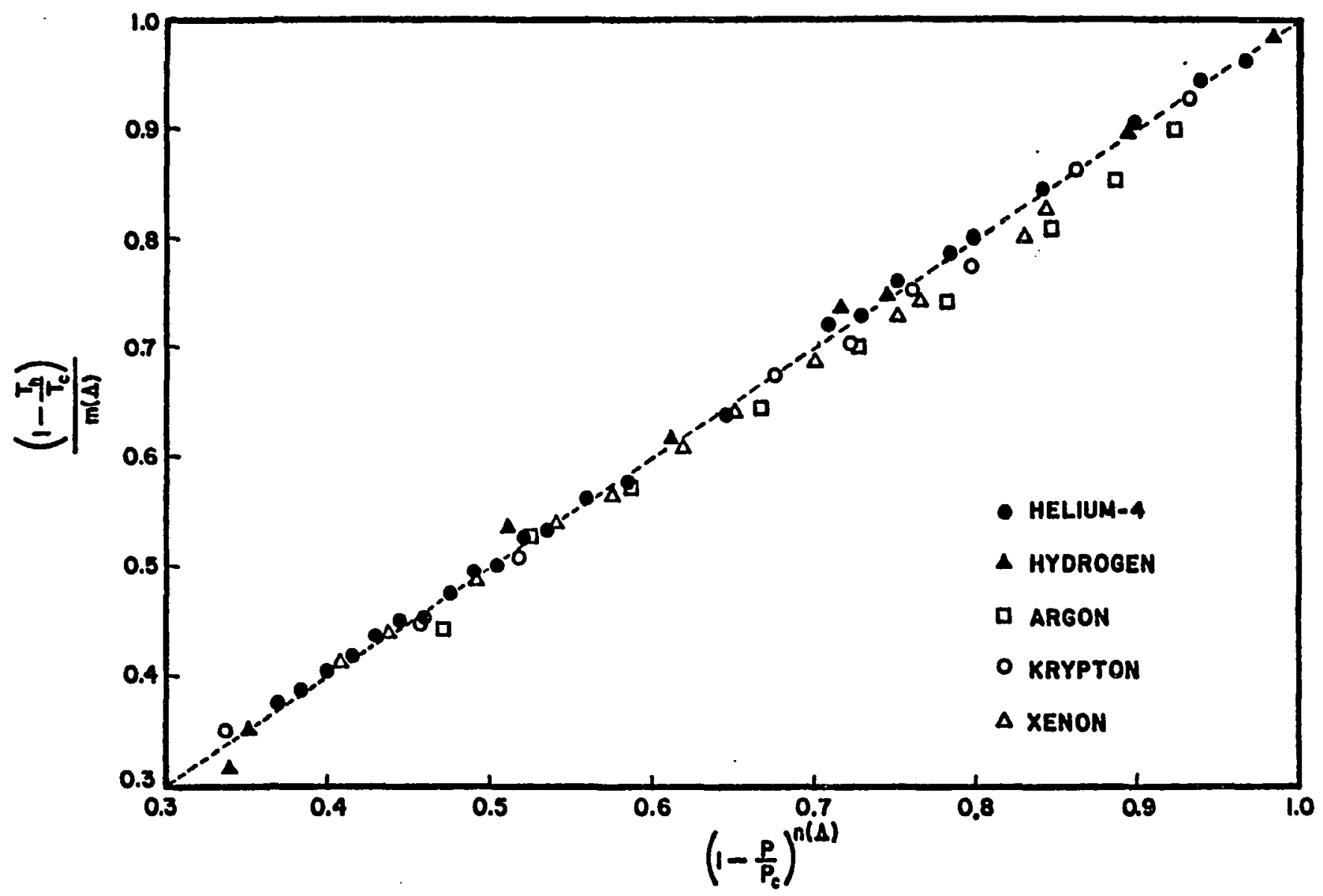

Figure 12. A plot of equation (3.17). A universal curve for the homogeneous nucleation temperatures of liquid noble gases. 
temperature of any hydrogen isotope with sufficient accuracy. A useful form of Eq. (3.14) in this respect is

$$
T_{h}^{*}=T_{c}^{*}\left[1-m(\Lambda)\left(1-\frac{P^{*}}{P_{c}^{*}}\right)^{n(\Lambda)}\right] .
$$

where $m$ and $n$ are given by Eqs. (3.15) and (3.16), or can be obtained from Fig. 11. The dashed lines in Fig. 10 represent the predictions of the homogeneous nucleation temperature for the hydrogen isotopes $D_{2}, \mathrm{HD}$, and $T_{2}$. It is otherwise difficult to obtain theoretical values for the nucleation temperature for these substances simply on the basis of the nucleation theory because the required thermophysical properties are not readily available. For instance, there is no surface tension data currently available for $T_{2}$. It should be pointed out that the quantum parameter $\Lambda$ used in the predictions for the heteronuclear isotope HD has been computed after Friedman (1962) as

$$
\Lambda_{\text {eff }}^{H D}=\Lambda_{\text {de Boer }}^{H D}\left[1+\frac{1}{6} \frac{\left(m_{1}-m_{2}\right)^{2}}{m_{1} m_{2}}\right]
$$

where $m_{1}$ and $m_{2}$ are the atomic masses of $H_{2}$ and $D_{2}$ respectively. 
CHAPTER IV

HOMOGENEOUS NUCLEATION IN LIQUID NITROGEN

\section{INTRODUCTION}

A metastable superheated state of a liquid becomes possible due to the potential of the liquid to attain a nonequilibrium state. The lifetime of such a metastable state depends on the extent of departure of the system from equilibrium, i.e. on the degree of superheating, and on the conditions to which the liquid is subjected. In the absence of external initiating influences such as solid surfaces, impurities or high energy radiation, a homogeneous liquid can be significantly superheated relative to the saturation temperature before a sufficiently large number of vapor nuclei can generate and grow spontaneously in the liquid. This process of spontaneous initiation of nucleation restricts further superheating of the liquid and is termed homogeneous nucleation. Thus, homogeneous nucleation in practice defines the maximum attainable superheat in a liquid. The kinetic theory of homogeneous nucleation (Becker and Doring 1935, Volmer 1939, Zel'dovich 1942, and Frenkel 1947) predicts the nucleation rate which depends exponentially on parameters related to the degree of superheating. Consequently, one obtains a sharp 
definition of the maximum attainable superheat in a liquid. Because of the extreme sensitivity of the nucleation rate on the superheat temperature of the liquid, one adopts the more or less conventional usage of referring to a single temperature, the homogeneous nucleation temperature.

In recent years the homogeneous nucleation temperature has been investigated extensively employing various techniques for a wide variety of organic liquids (see, for example, Blander and Katz 1977, Blander 1979, Skripov 1974). In all cases, fairly good agreement of the experimental data with the predictions from the kinetic theory of homogeneous nucleation has been observed. In contrast to the extensive studies done with organic liquids, the number and extent of such investigations involving cryogenic Iiquids have been limited (Skripov et al. 1977). Such studies in liquid nitrogen are completely lacking. The study of the maximum attainable superheat in liquids is essential for investigating the theoretical basis of homogeneous nucleation. From the practical point of view, a knowledge of homogeneous nucleation in liquid nitrogen is important in understanding the explosive boiling of liquid cryogens on a water surface (Enger et al. 1973). Moreover, since the homogeneous nucleation temperature depends exclusively on the intrinsic thermophysical properties of the fluid, its determination may prove to be essential in providing a reference temperature in heat transfer studies. In this context, homogeneous nucleation temperature data 
in nitrogen are necessary as a design parameter for systems in which liquid nitrogen is used as a coolant, e.g. cryoresistive transmission lines.

The main difficulty in the study of homogeneous nucleation involves the superheating of the liquid without interference due to the effects of heterogeneous nucleation. Consequently, the methods developed for studying homogeneous nucleation are based on enhancing the conditions which will render rates of homogeneous nucleation greater than for those of heterogeneous nucleation. One important condition in this respect is that the liquid effectively wet the substrate used for superheating. The smaller the contact angle, the easier it is to approach the maximum attainable superheat. In the case of liquid nitrogen such a condition is easily satisfied because of the small contact angle which liquid nitrogen forms with a solid surface (Bald 1973). The rising droplet technique developed by Wakeshima and Takata (1958) and Moore (1959) has been successfully used in the determination of the maximum attainable superheat for a large number of organic fluids by skripov and Ermakov (1964), Skripov and Sinitsyn (1964, 1967), Sinitsyn and Skripov (1968), Blander et al. (1971), and Porteous and Blander (1975). In this technique a drop of a volatile liquid is allowed to rise inside an immiscible heavier host liquid whose temperature is externally controlled. The interface between the two immiscible liquids provides good contact which renders the attainment of the homogeneous 
nucleation superheat limit by the liquid drop feasible. However, there are inherent restrictions involved in the rising droplet method, among which the requirement of a less volatile and denser immiscible host liquid is one. Unavailability of such a host liquid makes this technique unusable for studying liquid nitrogen.

However, Skripov and Pavlov (1970) and Pavlov and Skripov $(1965,1967,1970)$ introduced a new pulse heating technique for studying the kinetics of nucleation in strongly superheated liquids. A platinum wire, immersed in the test liquid, was pulse heated at a rate about $10^{6} \mathrm{~K} / \mathrm{s}$ using a single electric current pulse ranging from a low of $20 \mu \mathrm{s}$ to a high of $850 \mu \mathrm{s}$ duration. Under such conditions the liquid in the immediate neighborhood of the wire is strongly superheated and the nucleation is explosive in nature. Although this pulse heating technique was used only for organic liquids and water, the basis of the method was adopted for the case of liquid nitrogen. The first measurements of the homogeneous nucleation temperature of liquid nitrogen for reduced pressures $P / P_{c}$ ranging from .03 to .91 employing the transient superheating technique is reported herein. 


\section{APPARATUS AND EXPERIMENTAL PROCEDURE}

The apparatus consists essentially of a stainless steel cryostat of $7 \mathrm{~cm}$ inner diameter (Fig. 13) constructed specially to withstand pressures up to $10 \mathrm{MPa}$. A mechanical roughing pump is used to evacuate the space between the outer steel jacket and the inner stainless steel pressure vessel. Special high pressure hermetic seals are used for electrical feedthrough. A Setra system Model 204 pressure transducer with a $4 \frac{1}{2}$ digit digital pressure indicator is used to monitor the pressure inside the pressure vessel. The liquid $\mathrm{N}_{2}$ bath temperature is determined with a platinum resistance thermometer. A ring of ordinary soft soldering wire is used as a seal between the top flange and the stainless steel vessel. The soft solder provided an inexpensive and convenient substitute for indium wire seals and performed just as well at liquid nitrogen temperatures.

The test section consists of a platinum heating wire roughly $7 \mathrm{~cm}$ in length and $.102 \mathrm{~mm}$ in diameter attached to a spring loaded holder to keep the wire taut. The whole test section assembly is mounted vertically inside the pressure vessel. The heating wire also serves as its own thermometer. Platinum was chosen as the heating wire material because of its large temperature coefficient of resistance and high degree of linearilty in resistance vs. temperature over a wide range of temperatures. The 


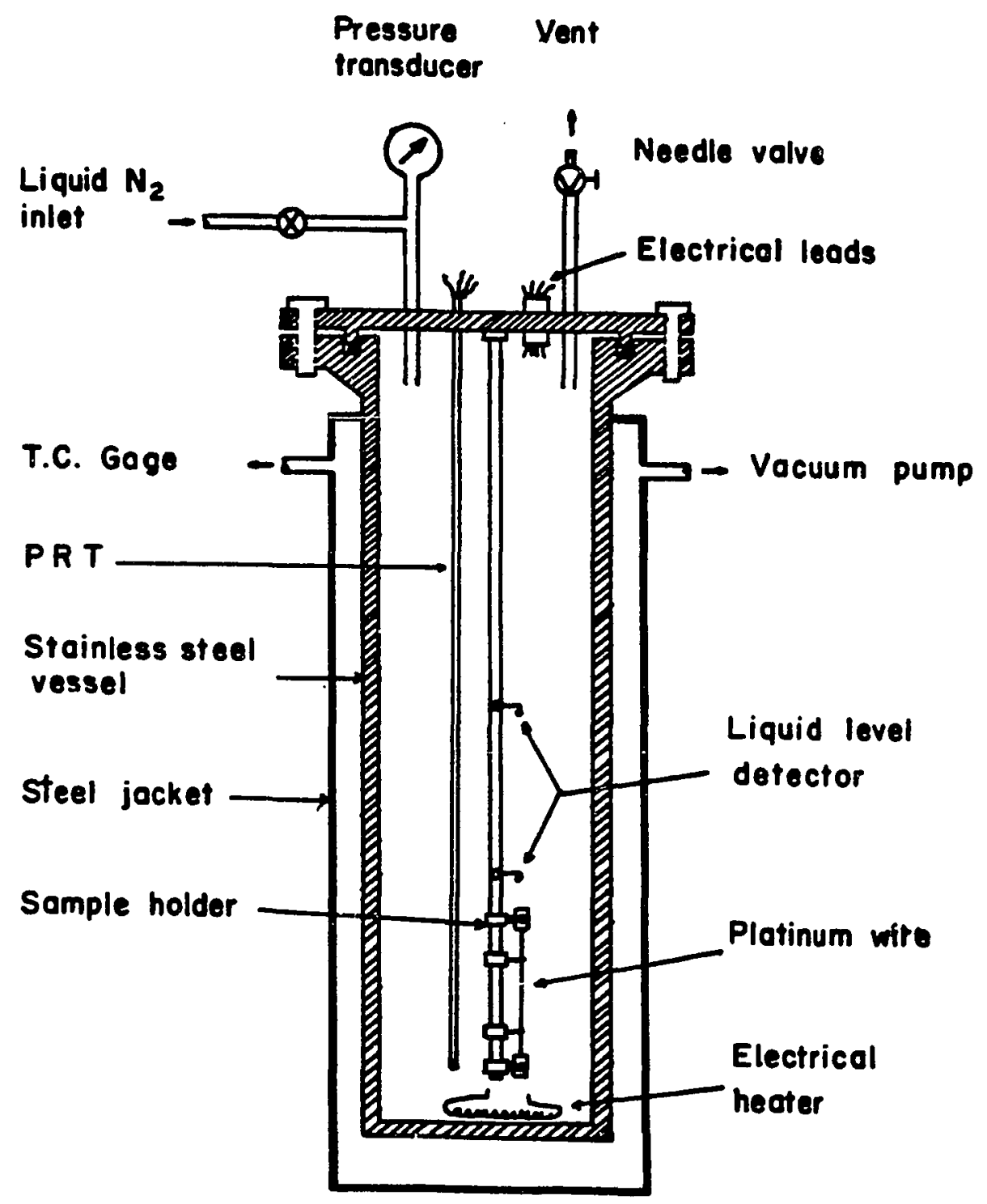

Figure 13. Schematic diagram of the cryostat assembly. 
electronic circuitry used and the procedure employed for the superheat temperature measurement employing the platinum heating wire is described in detail in Chapter VII and will not be repeated here.

The liquid nitrogen is transferred into the cryostat through the fill tube at the top. Two germanium diodes are used as liquid level detectors, one for indicating the starting level and the other one to indicate the level of the liquid when it reaches the top of the test section. After the cryostat is filled up to the desired level, the valve on the fill tube is shut off and the valve leading to the pressure transducer is opened. The pressure inside the cryostat vessel gradually builds up due to evaporation. The bath temperature $\mathrm{T}_{\mathrm{b}}$ and the bath pressure $\mathrm{P}$ are continuously monitored by feeding the analog outputs of the digital ohmmeter Kiethley Model 177, used for measuring the resistance of the platinum resistance thermometer, and the digital pressure indicator to an $X-Y$ recorder. The vapor pressure P-T curve for nitrogen was previously drawn on the paper used on the X - Y recorder in terms of the bath pressure and the resistance of the platinum thermometer. Through previous calibration of the platinum thermometer the resistance values are converted to temperature. Therefore, once properly calibrated, the $\mathrm{X}-\mathrm{Y}$ recorder output provides a continuous record of the thermophysical state of the liquid nitrogen inside the pressure vessel in terms of its pressure and temperature. A small 
heater at the bottom of the vessel is used to control the bath temperature in the initial stages of pressurization. First, the pressure is allowed to build up gradually close to the critical pressure of nitrogen. Then by controlling the heater power and the flow of vapor through the needle valve at the vent tube, the system is brought on to the saturation line previously drawn on the $\mathrm{X}-\mathrm{Y}$ recorder. This process takes between 1-2 hours. Once on the saturation line, the heater power is turned off and the vapor flow through the needle valve is adjusted so that the pressure drops at a uniform rate of roughly $600 \mathrm{~Pa}$ per minute. This ensures that the system will maintain itself on the saturation line. Thus, the actual experimentation begins at the highest pressure and ends at a lower pressure when the liquid nitrogen level reaches the bottom liquid level indicator. For the sake of simplicity, the system does not have any provision for filling the cryostat at high pressures. Consequently, several runs are made to cover the entire pressure range from atmospheric to about 918 of the critical pressure with each run taking about 4-5 hours. Such long run times are necessary to ensure that the bath pressure does not change more than $600 \mathrm{~Pa}$ during which the homogeneous nucleation temperature measurements are made. At the present time we are limited to a pressure of .91 $\mathbf{P}_{c}$ because above that pressure the magnitude of the observed superheat temperature is less than the accuracy of our temperature measurement. 
TRANSIENT SUPERHEATING TECHNI@UE

The simplest way to achieve superheating in a liquid is to heat a solid inside the liquid. However, a high degree of liquid superheat is not attained by this simple method because of the restrictive heterogeneous nucleation phenomenon at the solid-liquid interface. Surface irregularities such as grain boundaries, ledges, cracks, and scratches are responsible for heterogeneous nucleation. Interestingly, however, it has been observed in connection with transient heat transfer studies in liquid nitrogen (Oker and Merte 1978), in water (Sakurai and Shiotsu 1977), and in several organic liquids (Tolubinskiy et al. 1976, $1978,1979 a, 1979 b)$ that when the temperature of a solid surface in contact with these liquids is rapidly increased above the saturation temperature, heterogeneous nucleation occurs only after a finite time delay. This time delay is not of a constant value but decreases with increasing heating rate of the solid surface (see Chapter III and Sakurai and Shiotsu 1977). The onset of nucleate boiling occurs when the solid-liquid interface temperature reaches a certain superheat temperature, dependent upon the surface and liquid properties, surface microgeometry, and temperature gradient in the liquid. For sufficiently high heating rates natural convection in the liquid around the solid surface does not have time to develop and the superheating of the liquid adjacent to the surface takes place solely due to 
thermal conduction prior to the onset of heterogeneous nucleation.

Due to the finite delay time involved in the heterogeneous nucleation process, it is possible to attain a high degree of superheat for a thin liquid layer in contact with the solid surface under transient superheating conditions, if the heating rate is sufficiently high. Such a high heating rate can be easily achieved if a thin metal wire is electrically heated inside the liquid. Because of the small thermal capacity of the wire a significant portion of the electrical energy input is transmitted into the liquid and only a small fraction is distributed inside the solid wire. This transient superheating technique has been previously employed to determine the homogeneous nucleation temperature of liquid helium I (see Chapter III). This technique is well suited for studying cryogenic liquids. The low wetting angles and low surface tensions, characteristic of cryogenic liquids, make the presence of vaporfilled cavities on the solid surface highly unlikely. This results in longer delay times for nucleation and consequently relatively lower heating rates are sufficient. The low heating rates in turn obviate the necessity for using extremely thin heating wires as required in the case of organic liquids (skripov 1974). 
RESULTS AND DISCUSSION

We show typical sets of superheat temperature $\Delta \mathrm{T}$ vs. time curves at two representative bath pressures in Figs. $14 \mathrm{a}$ and $14 \mathrm{~b}$. In Fig. $14 \mathrm{a}$ note the kinks in the temperature rise curves occurring at the same superheat temperature $\Delta T_{h}$. The peak of the rounded overshoot corresponding to the next to lowest heating rate, curve $\underline{b}$, also occurs at the superheat $\Delta \mathrm{T}_{\mathrm{h}}$. Curves $\underline{\mathrm{b}}$ and $\underline{\mathrm{c}}$ illustrate the transition in the characteristics of the transient superheating curves from rounded temperature overshoots to the one which continually rises toward stable film boiling. As the bath pressure is increased, this transition moves to shorter time scales and roughly above .96 MPa only temperature overshoots with sharp peaks are observed as shown in Fig. $14 \mathrm{~b}$. It is worth pointing out the similarities in the characteristics of the transient temperature response curves in liquid helium and liquid nitrogen. The high pressure liquid nitrogen $\Delta T$ vs. time curves are almost identical to that of liquid helium (see Fig. 6). The sharp peaks occur at a constant superheat independent of the heating rate. At pressures lower than .5 MPa, the kinks in the $\Delta \mathrm{T}$ vs. time curves occur at a constant superheat. Such characteristic behavior in the transient temperature response was not observed by Pavlov and Skripov $(1965,1967,1970)$ in their pulse heating experiments. 

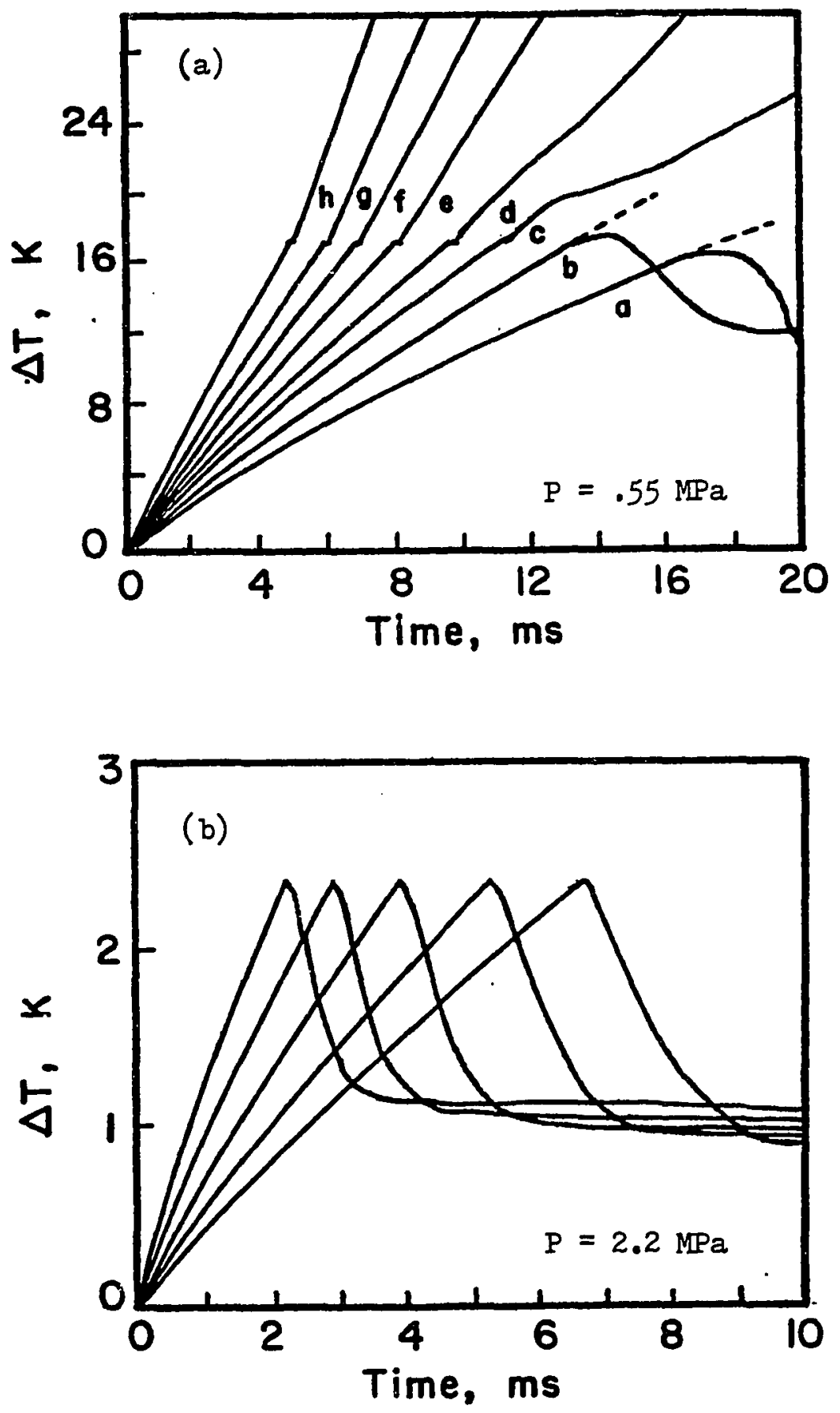

Figure 14. Superheat temperature $\Delta T$ vs. time. Transient superheat temperature curves are shown for two bath pressures in liquid nitrogen. Note in (a) the kinks in the temperature rise and in (b) the sharp peaks all occur at constant superheat temperatures. 
We interpret the sharp peak in the temperature overshoots and the kinks in the temperature rise curves at low pressures, both occurring at constant superheats, as indications of a sudden onset of homogeneous nucleation in the superheated liquid nitrogen layer in contact with the heating wire. At low pressures large superheating of the liquid is encountered. As previously mentioned, this superheating of the liquid is attained entirely due to heat transfer into the liquid by thermal conduction from the heating wire because the time scales for transient heating are much shorter than the long delay times involved in the onset of heterogeneous nucleation. This can be inferred from the excellent agreement between the observed transient superheat and the temperature rise predicted by assuming that the heat transfer is purely due to thermal conduction as illustrated by the dashed lines in Fig. 14a (see Appendix c). Therefore, during the superheating process, a large amount of thermal energy is accumulated in the superheated liquid nitrogen layer. When the homogeneous nucleation temperature is reached, a large number of critical vapor nuclei are formed spontaneously in the bulk of the superheated liquid layer. Their subsequent explosive growth is promoted by the high rate of vaporization associated with such high superheats. The behavior of the wire temperature at the onset of homogeneous nucleation is controlled by a combination of two factors, namely, rate of heat removal by vaporization and the thermal screening 
of the wire surface by the growing vapor film. At low pressures, the thermal screening of the wire surface following the onset of nucleation results in the continued rise of the wire temperature to a stable film boiling condition.

At high pressure, the break-off diameter of the bubbles is small (Kirichenko et al. 1975, Verkin et al. 1976) and the heat consumed for vapor formation is large, since the factor $L_{\rho} \rho_{v}$ increases significantly with temperature, where $I$ is the heat of vaporization and $p_{v}$ the vapor density. The latter factor contributes to a high rate of heat removal by vaporization of the liquid whereas the small break-off diameter reduces the chances of a vapor film formation around the heating wire. Consequently, at high pressures we see a sharp peak in the temperature overshoot indicating the onset of homogeneous nucleation with a subsequent cooling of the wire down to steady-state nucleate boiling. The required heating rates at high pressures are also much smaller, $100 \mathrm{~K} / \mathrm{s}$ at $.9 \mathrm{P}_{\mathrm{c}}$ compared to $3000 \mathrm{~K} / \mathrm{s}$ at atmospheric pressure. This is partly due to a relative shortage of nucleation sites, lower surface tension and lower contact angle of the liquid (Stepanov 1977) at higher bath pressures. The above factors reduce the influence of heterogeneous nucleation in transient superheating situations at high pressures.

We have used a laser beam from a He-Ne laser passing through the liquid layer adjacent to the wire surface to 
detect the rapid onset of homogeneous nucleation which we associate with the kink in the transient temperature rise of the wire. This experiment was performed separately in a glass Dewar. Figure 15 shows the correlation of the intensity signal from a photodetector, which detects the transmitted laser beam through the superheated liquid nitrogen layer, with the superheat vs. time curve at atmospheric pressure. The absence of random fluctuations in the photodetector signal prior to the kink confirms our view that heterogeneous nucleation is not involved at high heating rates. The sharp decrease in the transmitted laser beam intensity corresponds exactly to the kink and indicates that extremely rapid nucleation of vapor phase has taken place at that instant. The intensity of the scattered beam detected at $90^{\circ}$ to the direction of the incident beam also gives the same indications of rapid phase transitions. A further confirmation of this explosive nucleation is obtained from the acoustic signal from a submerged piezoelectric PZT-4 transducer. A sudden burst of acoustic energy was detected which coincided exactly in time with the occurrence of the kink in the transient temperature rise. The nucleation occurs with such rapidity, almost explosive in nature, that it is loud enough to be easily heard from outside of the Dewar.

In order to verify that the observed constant superheat temperatures $\Delta T_{h}$ are independent of the wire material, we have repeated the measurements with wires of different 


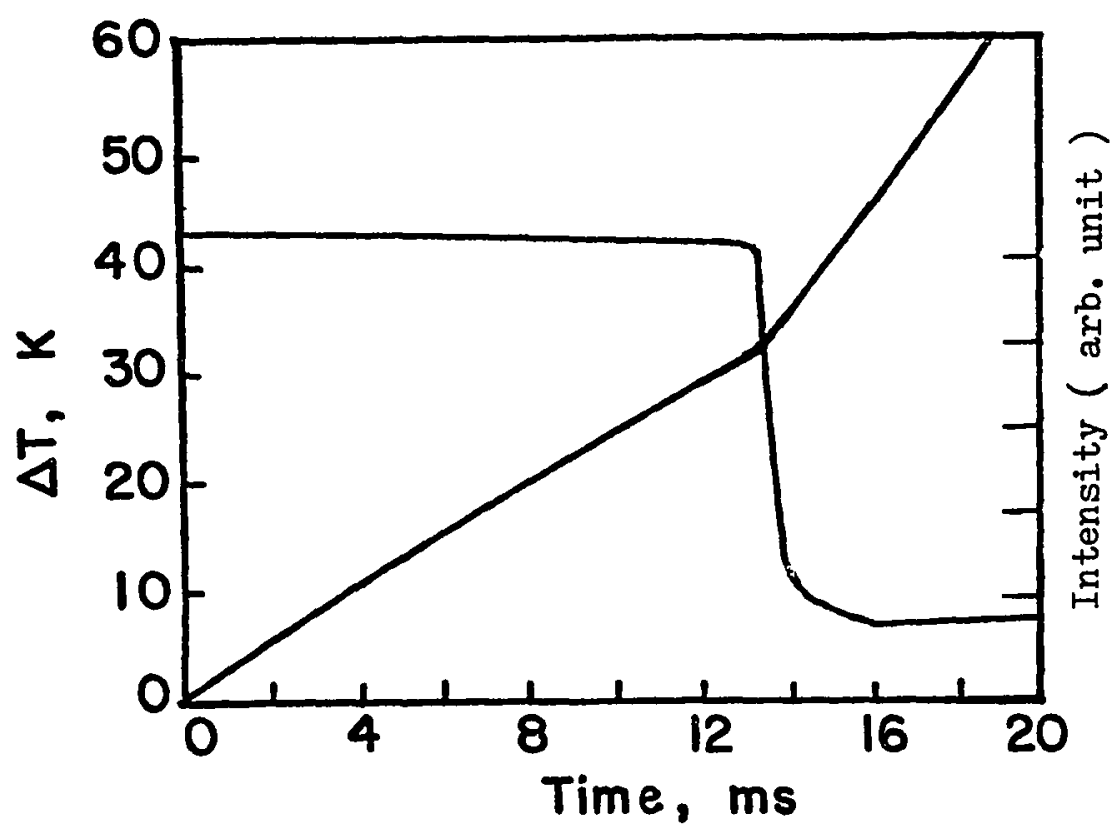

Figure 15. Superheat temperature and laser beam intensity vs. time. The bath pressure is atmospheric. Note the sharp decrease in the transmitted laser beam intensity occuring at the same time the temperature rise shows a kink. The superheat temperature corresponding to the kink is consistent with the estimate of homogeneous nucleation temperature based on the nucleation theory, equation (4.1). 
materials and dimensions. We have also repeated the atmospheric pressure measurements for both vertical and horizontal wires. In all cases the same value of the constant superkeat $\Delta T_{h}$ are observed within $\pm 0.1 \mathrm{~K}$, which is the accuracy of our temperature measurement.

It is worth mentioning at this point that homogeneous nucleation theory (Fisher 1948, Cole 1974) suggests that for cryogenic liquids such as liquid nitrogen, in which the wetting angle is very close to zero (Bald 1973), homogeneous nucleation in the bulk of the superheated liquid is more probable than heterogeneous nucleation at the wireliquid interface. This is true even for a surface which is perfectly smooth. As a result of this, it is relatively easy to observe homogeneous nucleation in cryogenic liquids. Finally, in Fig. 16 we show the experimental data on $T_{h}\left(T_{h}=T_{b}+\Delta T_{h}\right)$, cbtained from the transient superheating technique, as a function of the bath pressure in terms of their reduced values, $T_{h} / T_{C}$ and $P / P_{C}$. For comparison, we also show in Fig. 16 (solid line) the theoretical estimates for the homogeneous nucleation temperature based on the predictions from the nucleation theory. We employ the form recently given by Blander and Katz (1977) for the homogeneous nucleation rate

$$
J=N\left(\frac{2 \gamma}{\pi m B}\right)^{\frac{1}{2}} \exp \left[\frac{-16 \pi \gamma^{3}}{3 k T\left(P_{e}-P_{L}\right)^{2} \delta^{2}}\right]
$$




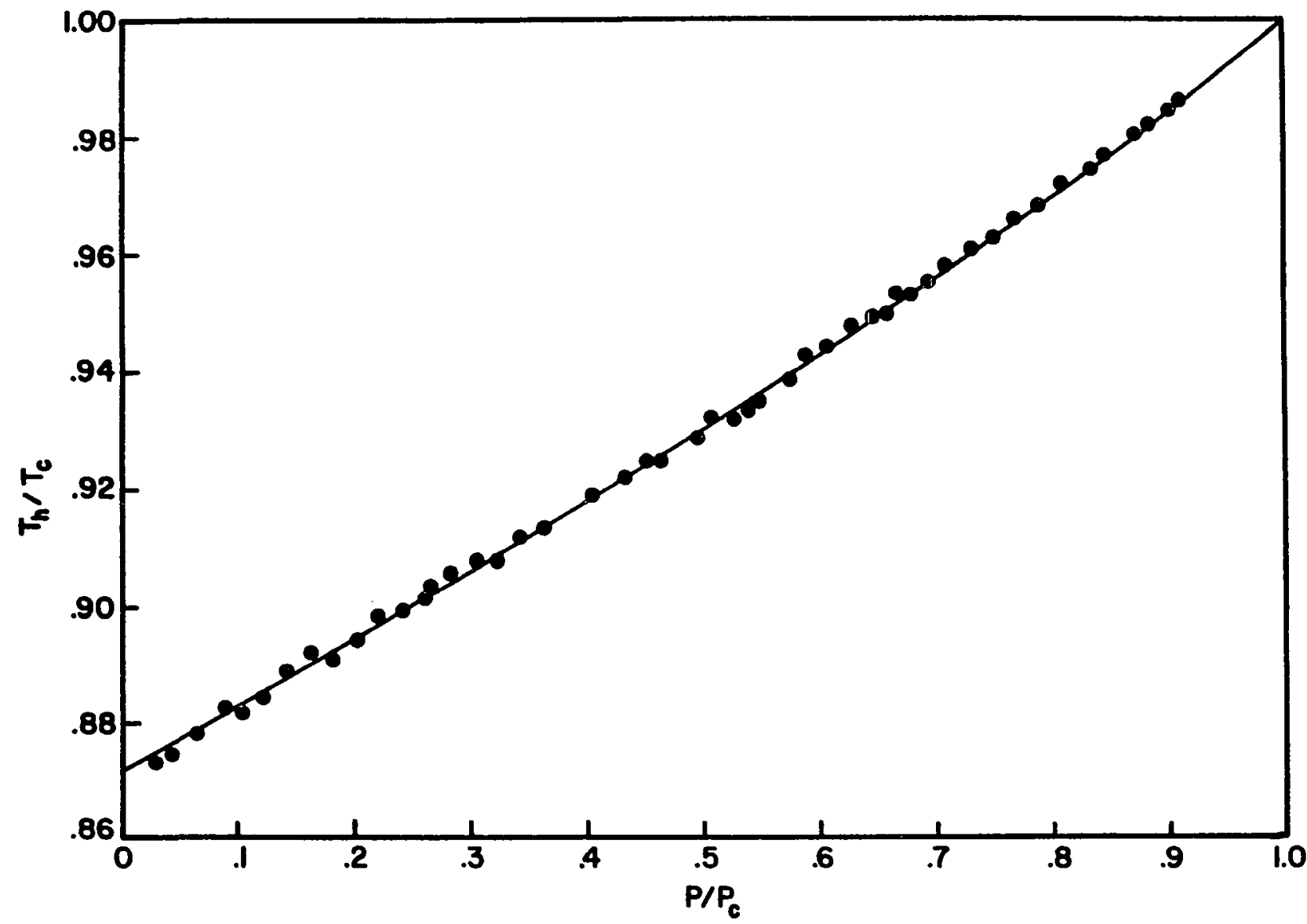

Figure 16. Scaled homogeneous nucleation temperature $T_{h} / T_{c}$ vs. scaled pressure $P / P_{c} \cdot$ The experimental temperatures $T_{h} / T_{c}$ (dark circles) are plotted as a function of the vapor pressure $P / P_{C}$ of the nitrogen bath. The solid line represents the prediction of homogeneous nucleation temperature from the nucleation theory for a nucleation rate $\mathrm{J}=10^{6}$. 
where the notation employed is that of the above reference. The solid line in Fig. 16 corresponds to a nucleation rate of $J=10$ nuclei/cm /sec. This nucleation rate is based somewhat arbitrarily on the heating rates employed in this study. Figure 17 illustrates how the homogeneous nucleation rate, obtained from Eq. (4.1), depends on the temperature at several bath pressures. The good agreement between the experimental data and the theoretical estimates of homogeneous nucleation temperature supports our interpretation that the kinks and the sharp peaks occurring at constant superheat temperature are indeed the result of a sudden onset of homogeneous nucleation in the superheated liquid in contact with the heater wire surface.

The experimental data on $\mathrm{T}_{\mathrm{h}}$ have been curve fitted by a function of the form

$$
\left(1-\frac{T_{h}}{T_{c}}\right)=0.1305\left(1-\frac{P}{P_{c}}\right)^{0.9115}
$$

with an accuracy of .2 per cent where $T_{c}$ and $P_{c}$ are the critical temperature and pressure of liquid nitrogen, respectively. The observed superheat temperature $\Delta T_{h}$ can be expressed within . 3 per cent in terms of the reduced bath temperature as

$$
\Delta T_{h}=200.1\left(1-\frac{T_{b}}{T_{c}}\right)^{1.65}\left[1-0.5394\left(1-\frac{T_{b}}{T_{c}}\right)\right], K
$$




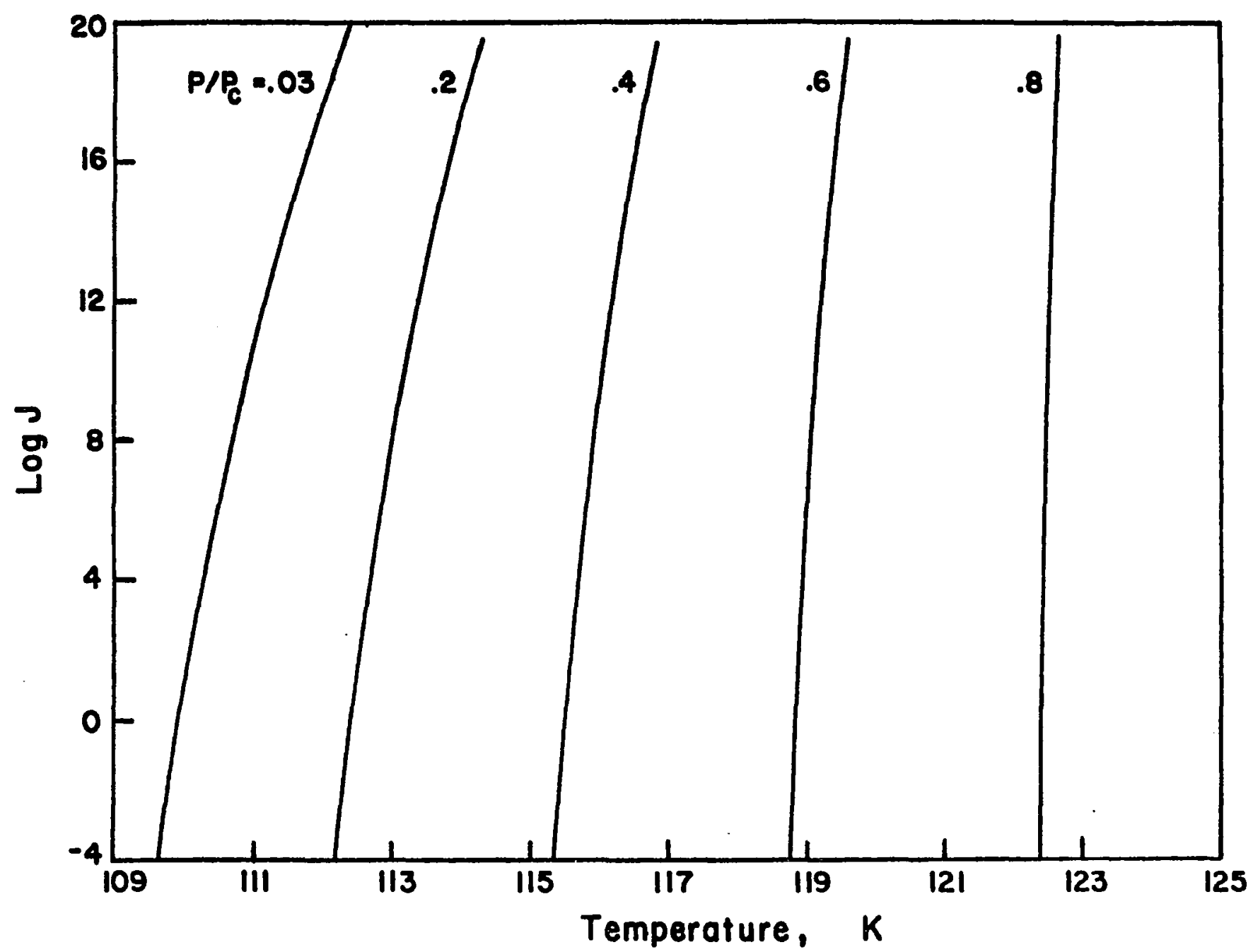

Figure 17. Temperature dependence of the nucleation rate. The temperature dependence of the nucleation rate $J$ in superheated liquid nitrogen is shown for five different bath pressures. $P_{c}$ is the critical pressure of nitrogen. 
COÑCLÜSION

It has been shown that the transient superheating technique developed by us is well suited for determining the homogeneous nucleation temperatures in cryogenic liquids such as liquid nitrogen. Low surface tension and small wetting angle make homogeneous nucleation inherently more probable in the bulk of the superheated liquid than heterogenecus nucleation at the solid-liquid interface. The existence of a delay time for the onset of nucleate boiling makes it possible to attain high superheat temperatures without the adverse influences of heterogeneous nucleation. The required heating rate ranged from $~ 100-3000 \mathrm{k} / \mathrm{s}$ depending on the bath pressure. Sharp temperature perturbations occurring at a constant superheat are taken as an indication of a rapid onset of homogeneous nucleation in a superheated liquid layer adjacent to the wire surface. These constant superheat temperatures are found to be in excellent agreement with the predictions from the homogeneous nucleation theory. 
CHAPTER V

\section{COMMENTS ON THE IIMITING SUPERHEAT TEMPERATURE}

OF LIQUID HELIUM I

The study of heat transfer in liquid helium is a subject of great practical interest from the point of view of applied superconductivity. In the past several years investigators have studied the nucleate boiling crisis condition in liquid helium. While the hydrodynamic theories, e.g. Kutateladze (1970), predict the peak nucleate boiling heat flux $q_{\max }$ with reasonable success, there does not yet exist any theory which says anything about the maximum nucleate boiling superheat temperature $\Delta \mathrm{T}_{\max }$ at departure from nucleate boiling. Investigations of nucleate pool boiling in he?ium with different heater surface material and surface conditions show a large dispersal in the $\Delta T_{\max }$ data. Apparently the thermal properties, microgeometry of the heater surface, and heating surface orientation, all have a significant influence on the nucleate boiling in helium. However, recently, Grigoriev et al. (1977) reported that the reduction of the heating surface roughness of stainless steel to 0.7 um brings about the complete disappearance of the nucleate boiling regime. They found that the incipience of boiling takes place, with consequent development of film boiling, at superheat 
temperatures of the order of $0.6 \mathrm{~K}$ which they claim corresponds to the limiting superheat temperature of liquid helium at atmospheric pressure. Kutateladze and Avksentyuk (1979) have termed the above process of vapor film formation on the heater surface without nucleate boiling as the 'third boiling crisis.' They also reaffirmed that the critical superheat temperature corresponding to the third boiling crisis at $\Delta \mathrm{T}_{\mathrm{cr} 3^{\prime}}$ should be such that $\Delta \mathrm{T}_{\max }<\Delta \mathrm{T}_{\operatorname{cr} 3}<\Delta \mathrm{T}_{\mathrm{L}^{\prime}}$ where $\Delta \mathrm{T}_{\mathrm{I}}$ is the limiting superheat temperature of liquid helium. Deev et al. (1977) assert that $\Delta \mathrm{T}_{\max }$ can be estimated as the liquid superheat $\Delta \mathbf{T}_{I}$. They calculated the $\Delta T_{I}$ values in two different ways: (1) from the van der Waals equation of state the spinodal curve which is the locus of the minima in the liquid isotherms, and (2) by extrapolating the isochoric lines on the pressure-temperature plane into the metastable region as first suggested by skripov (1974). The apparent agreement of $\Delta T_{\max }$ and $\Delta T_{I}$ values over a restricted range of reduced pressures was considered by the above investigators to be a verification of their claim. On the other hand, purdy et al. (1970) have reasonsed that the apparent lack of agreement of their $\Delta \mathrm{T}_{\max }$ with $\Delta \mathrm{T}_{\mathrm{I}}$ as estimated from van der waals equation of state is due to quantum mechanical modification of the principle of corresponding states.

The very important implication contained in the above works is that it is meaningful to expect that the limiting superheat temperature, an intrinsic property of the liquid 
only, could provide estimates for the peak nucleate boiling superheat temperatures in liquid helium which are significantly influenced by the heater material and surface conditions. In order to make a comparison between $\Delta T_{\max }$ and $\Delta \mathrm{T}_{\mathrm{I}}^{\prime}$ one needs to make the following two assumptions: (1) $\Delta T_{\max }$ represents the true temperature of the superheated liquid in the proximity of the heater surface, and (2) $\Delta T_{L^{\prime}}$ the spinodal temperature is the appropriate limiting superheat temperature for liquid helium. The purpose of this chapter is to point out that neither of the above assumptions is necessarily correct and that the apparent reported agreement (Deev et al. 1977) between $\Delta T_{\max }$ and $\Delta \mathrm{T}_{\mathrm{L}}$ is largely fortuitous. We shall discuss these points below.

We first examine assumption (1) as stated above. The measurement of $\Delta T_{\text {max' }}$ the maximum surface excess temperature, is made at the peak nucleate boiling heat flux conditions. At this stage of nucleate boiling the surface of the heated solid is only partly in contact with the liquid and a significant fraction of the surface is covered with vapor clusters due to coalescence of bubbles. Large positive temperature fluctuations of the surface directly beneath the vapor clusters have been observed by Swanson and Bowman (1974) in nucleate pool boiling of nitrogen. This fluctuation in temperature is primarily due to the difference in local heat transfer rates through the vapor and through the liquid. Thus, at any given instant, there is a 
distribution of local temperature variations over the heater surface. Consequently, the observed $\Delta \mathrm{T}_{\max }$ is a combination of both time and space average temperature of the entire heater surface and does not necessarily represent the temperature of the entire superheated liquid in the proximity of the surface or in momentary contact with it. The fact that the peak nucleate boiling superheat temperature does not in general represent the true superheat of the liquid is obvious from the data of Jergel and stevenson (1974) who report $\Delta T_{\max }$ values which correspond to temperatures higher than the critical temperature of liquid helium. The difference between the observed $\Delta T_{\max }$ and the average superheat temperature of the liquid helium adjacent to the heater surface should be dependent on the thermophysical properties of both the heater material and the 1iquid. As a result of this, the exact magnitude of the disagreement between $\Delta T_{\max }$ and $\Delta \mathrm{T}_{\mathrm{h}}$ can be expected to vary at different bath temperatures and also on different heater surfaces.

Before we examine the second assumption, a brief discussion of the limiting superheat temperature of a liquid is in order. In classical thermodynamics, stability criteria predict instability for a state if the isothermal compressibility $k_{T}$ becomes infinite or negative. This unstable limit defines the thermodynamic limit of superheat, also termed the spinodal line. As mentioned earlier, this limit is the locus of the minima in the liquid isotherms, i.e. the 
spinodal boundary, which satisfies the conditions $(\partial p / \partial v)_{T}=0$ and $\left(\partial^{2} p / \partial v^{2}\right)_{T}>0$, where $T$ is the temperature, $v$ is the volume and $p$ the pressure. The region between the spinodal line and the coexistence curve (saturation line) is the metastable region of a liquid, if the fluid exists in a single phase. The spinodal curve is generally supposed to represent the thermodynamic limit of stability of the metastable, uniform, one component phase. Unfortunately, there is no simple way to reliably determine this thermodynamic limit because, at present, there is no suitable equation of state available that properly represents the behavior of a liquid in the metastable region. But independently of this fact, this limit can never be reached physically, because this implies a fluid without fluctuations and microscopic structure. Volmer (1939), Becker and Doring (1935), Zeldovich (1942), and Frenkel (1947) developed a theory considering thermal fluctuations in the fluid, the so-called homogeneous nucleation theory which provides a kinetic limit of superheat. This kinetic limit of superheat incidentally is not defined by a single temperature but depends on the nucleation rate. However, because of the extreme sensitivity of the nucleation rate on the temperature of the superheated liquid one adopts a more or less conventional usage of referring to a single superheat temperature $\Delta \mathrm{T}_{\mathrm{h}^{\prime}}$ This kinetic limit of superheat, $\Delta \mathrm{T}_{\mathrm{h}^{\prime}}$ is always less than the thermodynamic limit of superheat $\Delta T_{L}$. 
As mentioned above, there is no straightforward way to determine the thermodynamic limit (spinodal) from the existing equations of state mainly because of the lack of evidence about their validity in the metastable region. However, a different approach to the spinodal may be possible. Skripov (1966) has shown that a plausible and simple estimate of the spinodal can be obtained by using the elementary hole liquid theory of Fürth (1941). Skripov and coworkers (Skripov 1974, Ermakov and Skripov 1970, Baidakov et al. 1975) verified the applicability of such an approach to the spinodal by showing the excellent agreement they obtained with the spinodal for argon and $n$-hexane as determined from the experimental $\mathrm{p}-\mathrm{v}-\mathrm{T}$ data in the superheated region. Fürth (1941) obtained an equation that essentially describes the spinodal in the T, P coordinates:

$$
P=P_{S}-1 \cdot 3 \gamma^{\frac{3}{2}}(k T)^{-\frac{1}{2}}\left\{1-\frac{v^{\prime}}{v^{\prime}}+\frac{3}{2}\left(\frac{v^{\prime}}{v^{\prime}}\right)^{2}\right\}^{-1}
$$

Here, $P_{\mathbf{S}}$ is the saturation vapor pressure, $\gamma$ is the surface tension; $v^{\prime}$ and $v^{\prime \prime}$ are the specific volumes of the liquid and the vapor on the saturation line corresponding to the temperature T. In Fig. 18 we show the spinodal obtained from Eq. (5.1) for $\mathrm{Ar}, \mathrm{H}_{2}$ and $\mathrm{He}^{4}$, the van der Waals' spinodal, and the superheat limit curve for $\mathrm{He}^{4}$ as obtained from the homogeneous nucleation theory. It should be pointed out that the He $\mathrm{H}^{4}$ spinodal in Fig. 18 is consistent with the spinodal determined with Deev et al. (1977) 


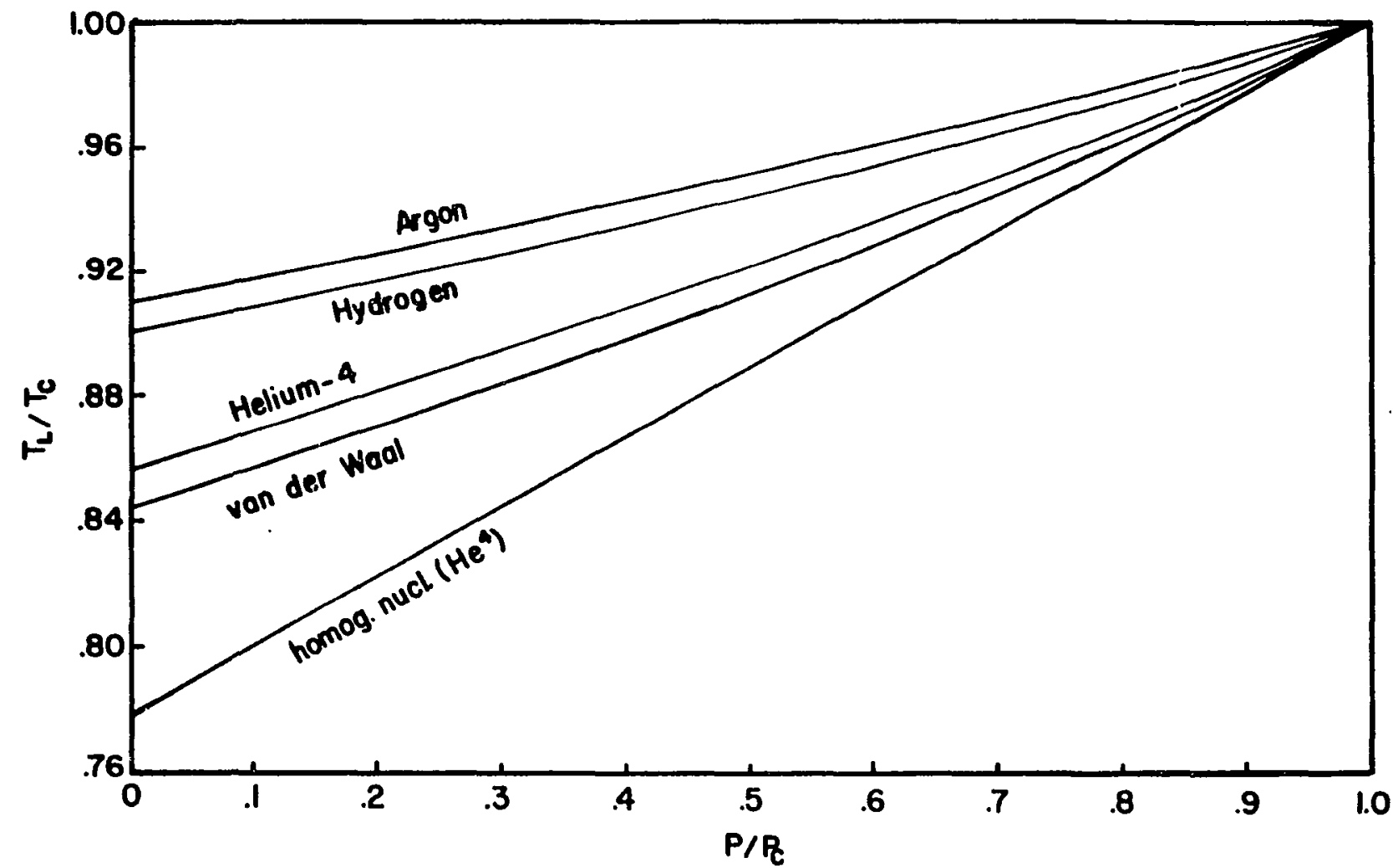

Figure 18. Spinodal for Argon, Hydrogen, and Helium -4 as determined from Furth's hole theory. Van der Waals' spinodal and the homogeneous nucleation limit of, superheat for He are also shown for comparison. 
following the method suggested by Skripov (1974) in which the spinodal is identified as the envelope of the family of isochores continued into the region of the metastable states. The relative shift in position of the spinodals for $\mathrm{Ar}, \mathrm{H}_{2}$, and $\mathrm{He}^{4}$ is due to a quantum effect as discussed in Chapter III. It is obvious from Fig. 18 that the kinetic limit $T_{h}$ is lower than the thermodynamic limit $T_{L}$ as mentioned earlier. It is also evident that the validity of using van der Waals' equation of state to determine the spinodal is in general totally unjustified for purposes of quantitative evaluations. Further evidence for this comes from the fact that the homogeneous nucleation temperature of liquid noble gases such as Argon, Krypton, and Xenon are significantly higher than the estimated spinodal temperatures of a van der Waals' fluid, contrary to what shoula be expected.

It is pertinent at this point to ask which limit of superheat, kinetic or thermodynamic, provides a realistic representation of the experimentally observable limiting superheat temperature of a liquid. As discussed above the thermodynamic approach does not take into account the heterophase fluctuations in the system which assume a special role in the metastable phase. In view of present knowledge of nucleation phenomena it appears that the time during which a superheated metastable liquid can exist as a homogeneous system is limited. Under conditions of appreciable superheating of the liquid, the lifetime for 
the decay of the metastable state through homogeneous fluctuations, decrease rapidly to unobservable, sublaboratory times even before the spinodal is reached. For instance, in the case of liquid helium, the estimated lifetime of the metastable state, based on nucleation theory is of the order of $10^{-26}$ second at the spinodal (this result is somewhat arbitrary, since it is based on the assumptions that the conventional mechanism of nucleation acts on the stability limit of the phase, and that liquid helium is a van der Waals' fluid). It is obvious that the spinodal could not be observed and its definition would be purely a matter of convention. The experimentally attainable maximum superheat of a liquid is limited by the kinetic limit of superheat (homogeneous nucleation) which is always lower than the thermodynamic limit. Thus it is reasonable to consider the homogeneous nucleation temperature, $T_{h}$ ' as the appropriate measure of the experimentally realizable maximum superheat of a liquid. It would have been more mearingful to compare $\Delta \mathrm{T}_{\max }$ with $\Delta \mathrm{T}_{\mathrm{h}}$ and not with $\Delta \mathrm{T}_{\mathrm{L}}$ as all the investigators mentioned in this chapter have done. It is important to note that the kinetic limit of superheat which can be determined experimentally is likely to be realized in liquid helium under transient superheating conditions. The first such measurements of the limiting superheat temperature $\Delta \mathrm{T}_{\mathrm{h}}$ of liquid helium were previously reported by the author (Brodie et al. 1977) using a transient magnetoresistive thermometry technique described 
in Chapter II. The liquid helium in contact with a transient heater-thermometer was superheated by thermal conduction up to its limiting superheat temperature $\Delta \mathbf{T}_{\mathrm{h}}$ before appreciable heterogeneous nucleation could take place. Similar experiments with liquid nitrogen have also been carried out and will be reported separately. Such transient heating experiments were also performed by skripov (1974) specifically for measuring the limiting superheat temperatures of several organic liquids.

In Fig. 19 we present experimental data on $\Delta T_{h}$, recent data on $\Delta \mathrm{T}_{\max }$ by several investigators, the van der Waals' spinodal curve $T_{L^{\prime}}$ and the theoretical estimates of the limiting superheat temperatures over a wide range of bath temperatures in liquid helium I. The van der Waal' spinodal curve is shown only to serve as a qualitative reference point. The experimental data on $\Delta T_{h}$ which were obtained by our transient measurements have been expressed in terms of reduced temperature $T / T_{C}$ by

$$
\Delta T_{h}=\Delta T_{\text {ref }}\left(1-\frac{T}{T_{c}}\right)^{1.529} ;\left(\Delta T_{\text {ref }}=4.268 \mathrm{~K}\right)
$$

where $\mathbf{T}_{C}$ is the critical temperature of liquid helium. Although helium is a quantum liquid, nevertheless, we assume the use of the so-called 'classical' nucleation theory for estimating the limiting superheat temperatures in helium to be valid. The excellent agreement of the experimental data on $\Delta T_{h}$ with the theoretical predictions confirm our 


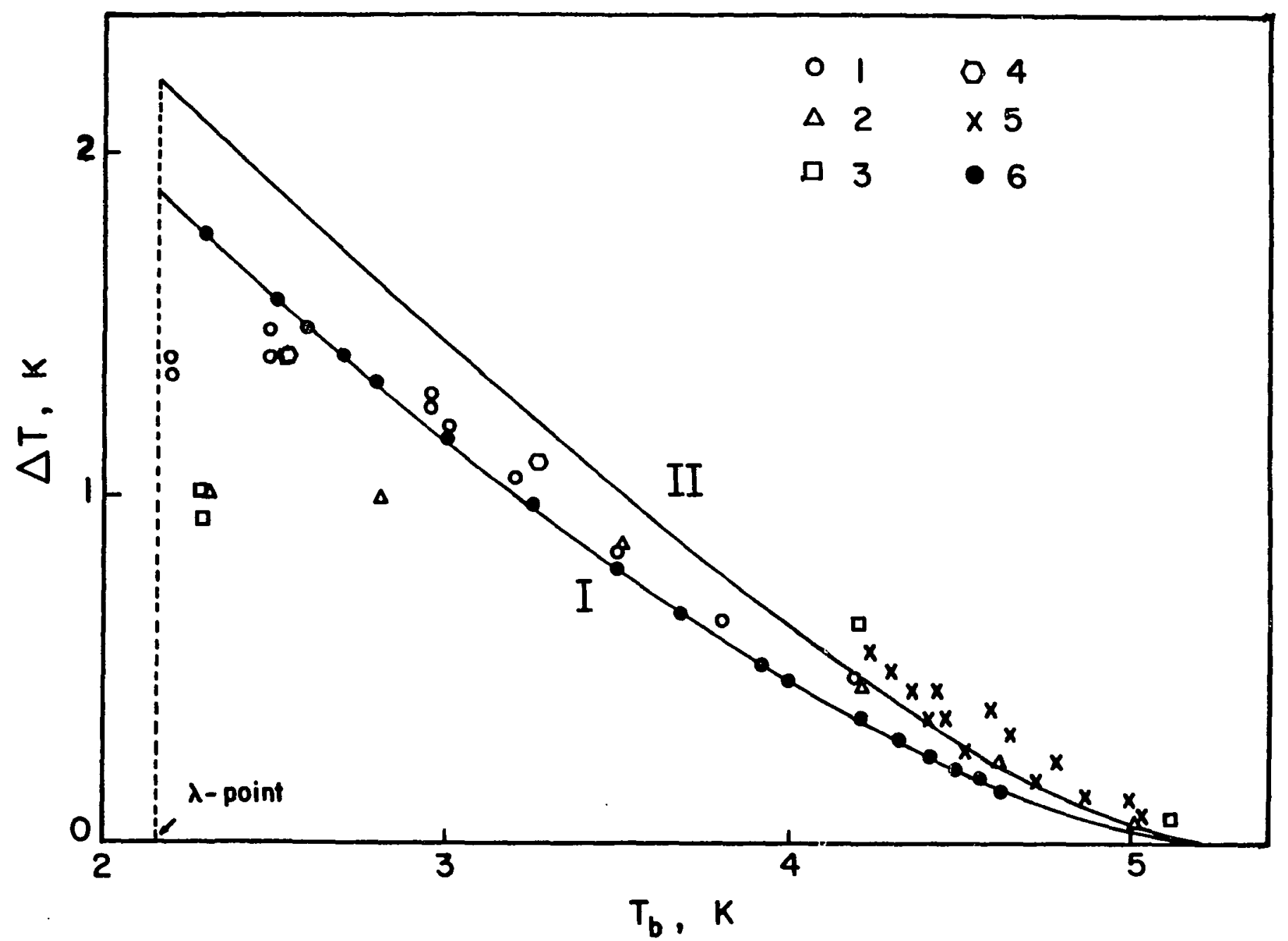

Figure 19. Superheat temperature at critical heat flux as a function of bath temperature. $\Delta T, 1$ - Purdy et al.(1970), 2 - Bewilogua et al. (1975), 3 - Lyon (1965), 4 - Goodling and Irey (1969), 5 - Deev et al. (1977), 6 - present study (Chapter III).

Curve I corresponds to the theoretical predictions of the limiting superheat temperature $\Delta \mathrm{T}_{\mathrm{h}}$ based on the nucleation theory. Curve II corresponds to the spinodal curve $\Delta \mathrm{T}_{\mathrm{I}}$ determined from van der Waals' equation of state. 
assumption. Our results are consistent with the quantum theory of nucleation of Lifshitz and Kagan (1972) who predict that nucleation due to quantum tunneling is not expected to occur above a threshold temperature of $\sim 0.3 \mathrm{~K}$ which is well below the helium I range. Incidentally, one is likely to observe the tunneling nucleation, if at all, only in tensile strength experiments and not in superheating experiments.

The maximum nucleate boiling superheat temperature data as presented in Fig. 19 are obtained by various investigators with different heater materials and surface conditions. Although these data from different investigators are not entirely in quantitative agreement with each other, they all show a definite pattern in relation to $\Delta \mathrm{T}_{\mathrm{h}^{\prime}}$ curve $\mathrm{I}$. We find that in the temperature interval from the superfluid transition temperature $\mathrm{T}_{\lambda}(2.17 \mathrm{~K})$ to roughly $3 \mathrm{~K}$ all the $\Delta \mathrm{T}_{\max }$ values fall below $\Delta \mathrm{T}_{\mathrm{h}}$. In the middle range between $3 \mathrm{~K}$ to $4 \mathrm{~K}$, all the $\Delta \mathrm{T}_{\max }$ data presented are reasonably in agreement with $\Delta \mathrm{T}_{\mathrm{h}}$ although being slightly higher in value. However, in the highest temperature range, from roughly $4 \mathrm{~K}$ to the critical temperature $(5.2 \mathrm{~K})$, all the maximum nucleate boiling superheat data are uniformly higher than $\Delta T_{h}$ and by pure coincidence are in general agreement with the superheat temperature $\Delta \mathrm{T}_{\mathrm{L}}$ corresponding to the van der Waals' spinodal, curve II in Fig. 19. Incidentally, in terms of reduced pressure, this highest temperature interval 
corresponds to a wide range roughly from 0.5 to 1 . This fortuitous agreement between the $\Delta T_{\max }$ data with $\Delta T_{L}$ has led Deev et al. (1977) to the erroneous conclusion that the maximum nucleate boiling temperature can be estimated as the liquid helium superheat. As we had mentioned earlier, such a comparison with the liquid spinodal $\left(\Delta T_{L}\right)$ is unrealistic and apparently meaningless.

In contrast to the large number of nucleate boiling data available at and above $4.2 \mathrm{~K}$, the availability of such data in the temperature range between $3 \mathrm{~K}$ to $4 \mathrm{~K}$ is severely limited. This range is particularly interesting because the data of Purdy et al. (1970) appear to be in consistent qualitative agreement with the limiting superheat temperature of liquid helium, curve I in Fig. 19. One isolated data point by Bewilogua et al. (1975) within that range is also internally consistent. Interestingly, however, Purdy et al. (1970) raised the question of quantum mechanical modification of the principle of corresponding states to partly explain the lack of agreement of their $\Delta T_{\max }$ data with the van der Waals' spinodal, curve II. In raising this question they imply that if an appropriate equation of state were available for a quantum liquid such as helium which would properly represent the metastable liquid state, then the spinodal curve $\left(\Delta T_{L}\right)$ obtained from it should have been expected to be in agreement with their nucleate boiling data. In light of our previous discussion related to the experimentally realizable superheat limits 
of a liquid, it is clear that their assertion has little justifiable basis. The quantum effects on the principle of corresponding states as applied to the case of limiting superheat temperatures for the liquid noble gases have been discussed in detail in Chapter III.

The reduction in the $\Delta T_{\max }$ data in comparison with $\Delta \mathbf{T}_{h}$, as the $\lambda$ - point is approached, is qualitatively explained by Kutateladze and Avksentyuk (1979) by their model based on the cavitational mechanism of vapor film formation in the transition boiling regime. They set the conditions such that $\Delta \mathrm{T}_{\max }<\Delta \mathrm{T}_{\mathrm{cr} 3}<\Delta \mathrm{T}_{\mathrm{h}}$. Although in the Iimited temperature range between $\mathrm{T}_{\lambda}$ and $3 \mathrm{~K}$ the above condition is true, above $3 \mathrm{k}$ the experimental data do not support their assertion. Contrary to their statement, we find that above $3 \mathrm{~K}$ both $\Delta \mathrm{T}_{\max }$ and $\Delta \mathrm{T}_{\mathrm{crs}}$ are in general higher than $\Delta \mathrm{T}_{h}$. It should be mentioned that the value of $0.6 \mathrm{~K}$ for $\Delta \mathrm{T}_{\mathrm{Cr} 3}$ at atmospheric pressure as reported by Grigoriev et al. (1977) is open to question because it is highly unlikely that even on a smooth surface liquid helium can exist in a metastable state so far beyond its limiting superheat temperature of $0.35 \mathrm{~K}$.

It appears at the present time that it is not likely that the peak nucleate boiling temperature in helium can be estimated from the liquid superheat limit alone without incorporating the effects due to the surface conditions and the thermophysical properties of the heater material as well as those of the liquid. For any quantitative 
theoretical prediction for the superheat temperature at nucleate boiling crisis, a knowledge of the true limiting superheat temperature of liquid helium may prove to be essential. 


\section{CHAPTER VI}

\section{IIGHT INDUCED ENHANCEMENT OF TRANSIENT HEAT TRANSFER FROM A SOLID INTO LIQUID HELIUM}

\section{INTRODUCTION}

The economic justification for MHD, fusion, energy storage, and in fact most large magnet systems, rests upon the feasibility of large superconducting magnets. For this reason a considerable amount of work is currently underway in this country and elsewhere in developing the engineering technology of such systems. Underlying many of the engineering problems is the extreme susceptibility of superconductors to small heat inputs; one can not even ignore the thermal energy generated by friction during minute position shifts of the windings. The most common type of superconducting magnet is cooled by pool boiling in liquid helium where the conductors are wound with channels allowing free circulation of liquid helium around them to facilitate cryostatic stabilization. If heat is generated in the conductor either as a result of a flux jump or mechanical instability, the heat produced can be transferred to the helium at a rate that limits the temperature rise of the superconducting winding. The rate of heat transfer to liquid helium is normally considered to be limited by 
nucleate pool boiling, this being the most efficient mode of heat transfer under steady state conditions. However, it has been gradually recognized that the steady state analysis of superconducting magnets must be modified by applying transient heat transfer parameters. For these reasons increasing interest has been shown in studying transient heat transfer in 1iquid helium (Jackson 1969, Tsukamoto and Kobayashi 1975, Brodie et al. 1977, Steward 1978, Iwasa and Apgar 1978).

Recently, in connection with our studies of transient heat transfer in liquid helium, we have observed a new effect: a marked enhancement of the transient heat transfer from a solid, the bismuth heater-thermometer (Brodie et al. 1978, also see Chapter II), to liquid helium due to the application of visible light to the bismuth-helium system. Our studies show that when the liquid in contact with the surface of a heater-thermometer has been rapidly superheated to a temperature high enough that nucleate boiling is either imminent or actually occurring, the flashing of a single strobe light pulse of about $40 \mu \mathrm{s}$ duration through the unsilvered sides of the glass Dewar causes a sudden large drop in the bismuth crystal temperature. The effect is striking: our measurements show that in three milliseconds the transient temperature can drop by as much as 75 per cent of the total superheat. The increase in the transient heat transfer occurs very rapidly with about 
90 per cent of the temperature drop typically occurring in under a millisecond.

\section{EXPERIMENTAL DETAIIS}

Most of the experimental details are similar to those presented in Chapter II. See Figs. 2 and 3 in Chapter II for a description of the crystal mounting and for the schematic circuit diagram. The light pulse whose wavelength covered the visible spectrum was provided by a Strobotac strobe (not shown in the above figures). This strobe has three settings for the light pulse intensity: Low, Medium, and High, which are in the proportion of 1:8.25:29 as determined by a phototransistor. The duration of the light pulse from this strobe for all intensity settings is about $40 \mu \mathrm{s}$. The flash tube of the strobe was positioned about $35 \mathrm{~cm}$ from the crystal. The strobe was triggered by a trigger output from the pulse generator which sends a step-function heating curren't to the crystal. A variable pulse delay circuit (not shown in Fig. 3 , Chapter II) was placed between the pulse generator and the strobe so that the light pulse from the strobe could be delayed by any required amount after the heating current was turned on. A phototransistor with a 1 ns response time was employed to detect the light pulse and to mark on the storage scope at the time it occurred. These time marks are shown as arrows in Fig. 20 to 26 . In addition to the 
Strobotac, occasionally a high intensity, General Electric type 1532-D Strobolume was also used. The Strobolume is triggered in turn by the strobotac unit. The strobolume pulse duration is of the order of $3 \mathrm{~ms}$. A polaroid filter was placed between the strobolume and the crystal to conveniently control the intensity of the light falling on the bismuth helium system. Although the relative intensities of the light pulses could be determined by the phototransistor, the absolute light intensities could not be accurately measured.

\section{DESCRIPTION OF LIGHT-PULSE INDUCED ENHANCEMENT}

The light effect can best be described by referring to Figs. 20 and 21 which show the change in bismuth crystal temperature as a function of time after the application of a step function electric heating pulse. In all cases to be discussed the initial bath temperature was $4.2 \mathrm{~K}$. For purposes of comparison with the light-affected curves which follow, Fig. 20 shows a typical curve of superheat temperature $\Delta T$ as a function of time $t$ when no light is applied. Note that the superheat temperature at first rises rapidly to a maximum, and then falls back to an intermediate steady-state nucleate boiling value. The initial stage of the temperature rise is in good agreement with the prediction of the heat conduction equation (Lurie and Johnson 1962), and is consistent with the assumption that during that initial period zeither convection nor nucleate 


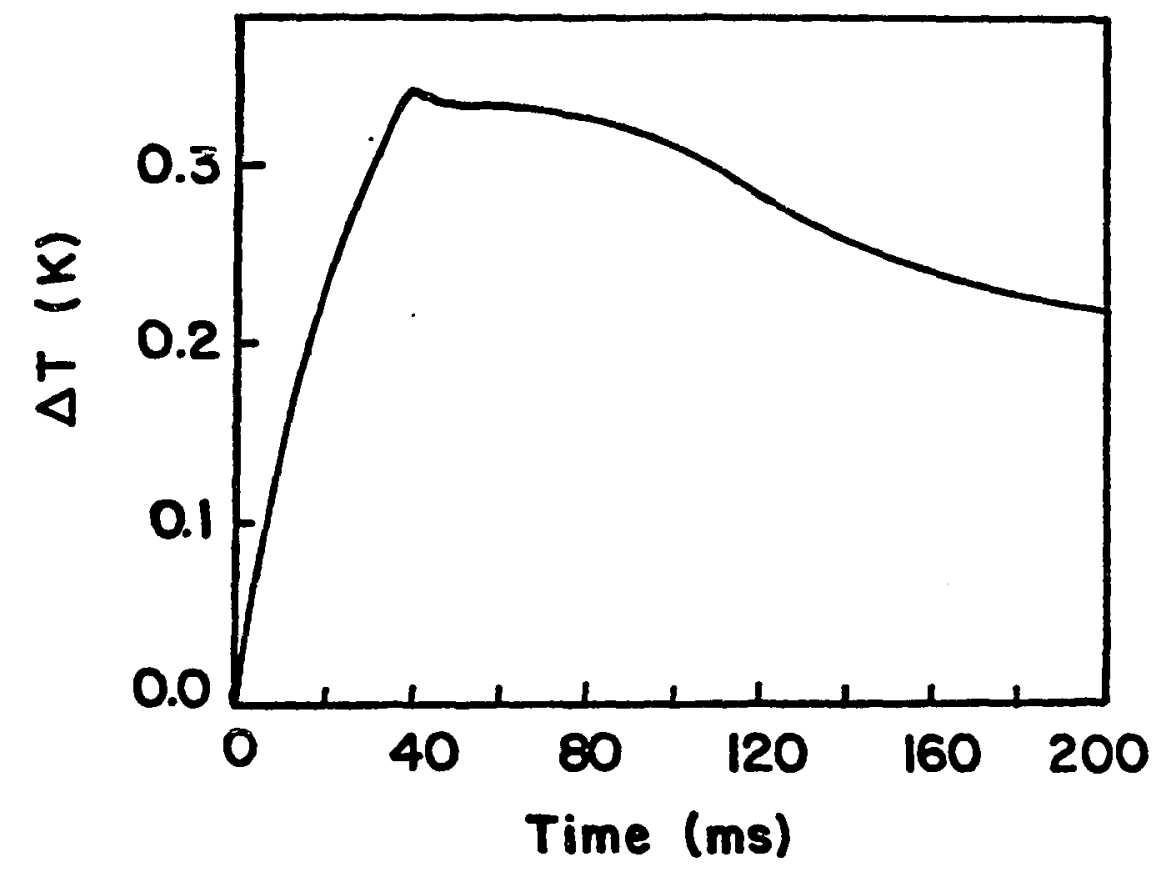

Figure 20. Superheat temperature $\Delta \mathrm{T}$ vs. time. The step function electric power is applied at time $t=0$. No light is applied to the system.

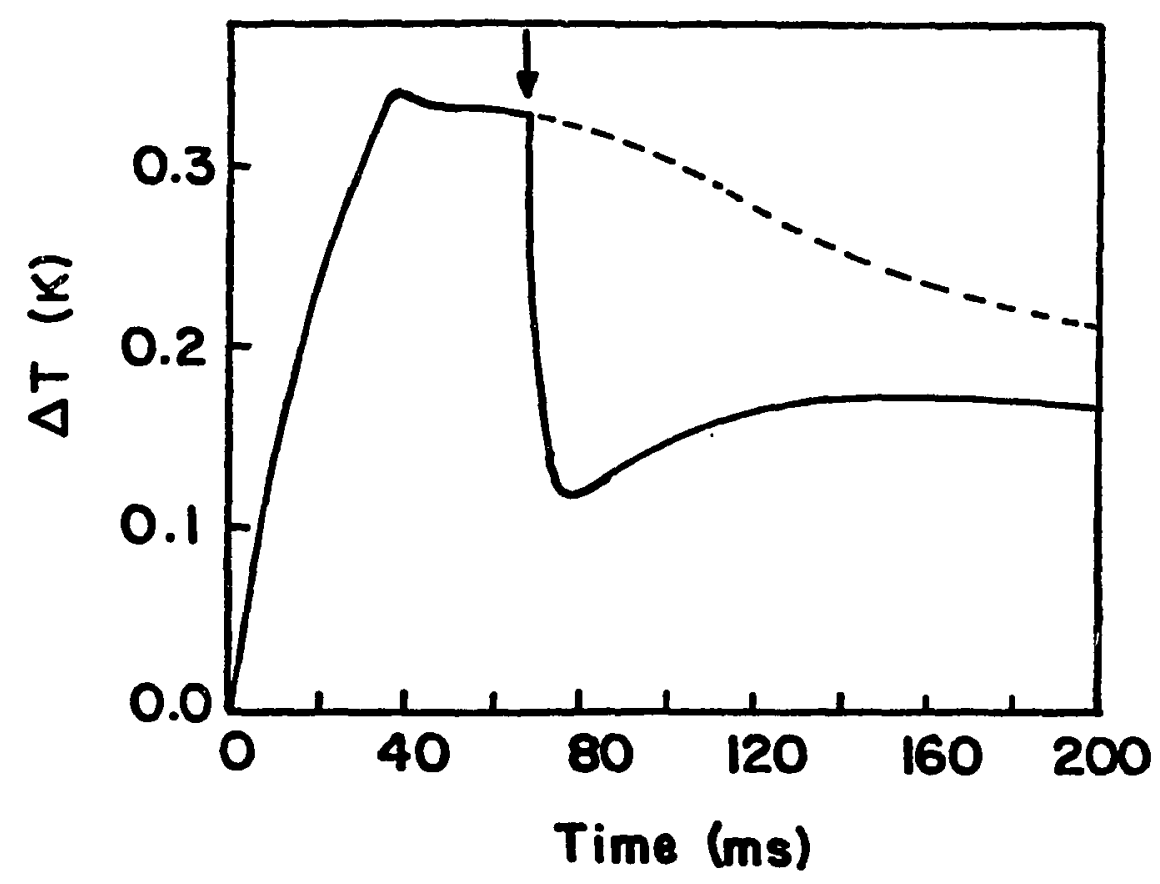

Figure 21. Effect of pulsed light on the $\Delta T$ vs. time curve. A $40 \mu \mathrm{s}$ duration light pulse is applied at the time indicated by the arrow. For the purpose of comparison, the dotted line shows the curve obtained when no light is applied 
boiling has had time to develop. The occurrence of the sharp temperature peak signals the sudden onset of homogeneous nucleation, followed by a subsequent relaxation to steady-state nucleate boiling (Brodie et al. 1977, Sinha et al. 1977, Semura et al. 1979, see also Chapter III).

\section{Heat transter enhancement}

Figure 21 shows the effect of a $40 \mu \mathrm{s}$ pulse of visible light applied at the time marked by the arrow. Note that the temperature drops dramatically when the light pulse is applied, indicating a sudden enhancement of heat transfer due to the action of light. The dotted line in Fig. 21 is the continuation of the no-light curve of Fig. 20 and is shown for comparison.

It should be pointed out that the observed effect cannot be simply the result of photoconductivity. Since the magnetoresistance of bismuth decreases with increasing temperature, the temperature excursion signal which is triggered by the light pulse represents an increase of resistance rather than the decrease which would be expected of a photoconductive effect. Moreover, as explained in the next paragraph, there is no effect if the light is flashed before a certain threshold temperature is reached.

Figures 22 and 23 show composite records of several different experiments in which the light pulse was applied at different times. The times of application of the light pulses are indicated by the arrows in both figures. Note 


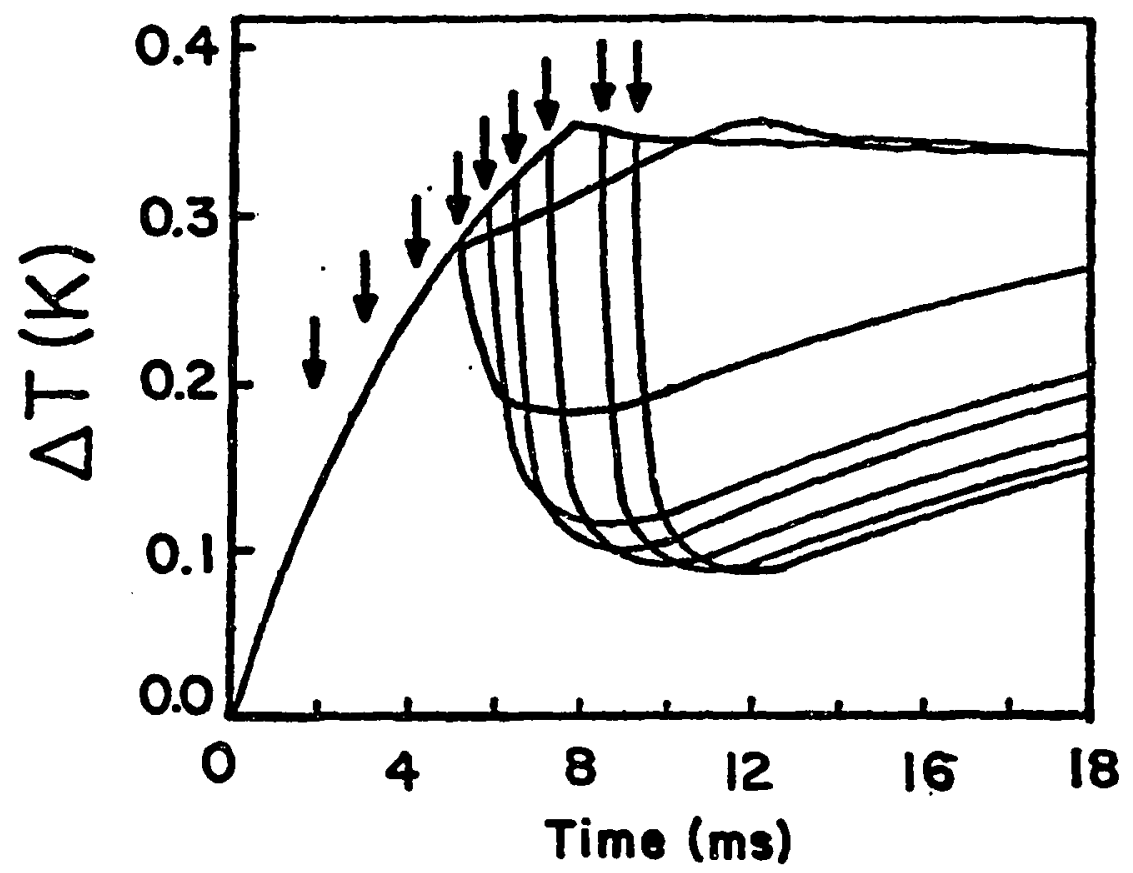

Figure 22. Effect of pulsed light on the transiert superheat temperature characteristics. The figure shows a composite record of nine separate experiments with the light pulse being applied at successively earlier times. The arrows indicate the times of application of the light pulses. Note that there is no effect of light below a threshold superheat temperature value of about $0.3 \mathrm{~K}$.

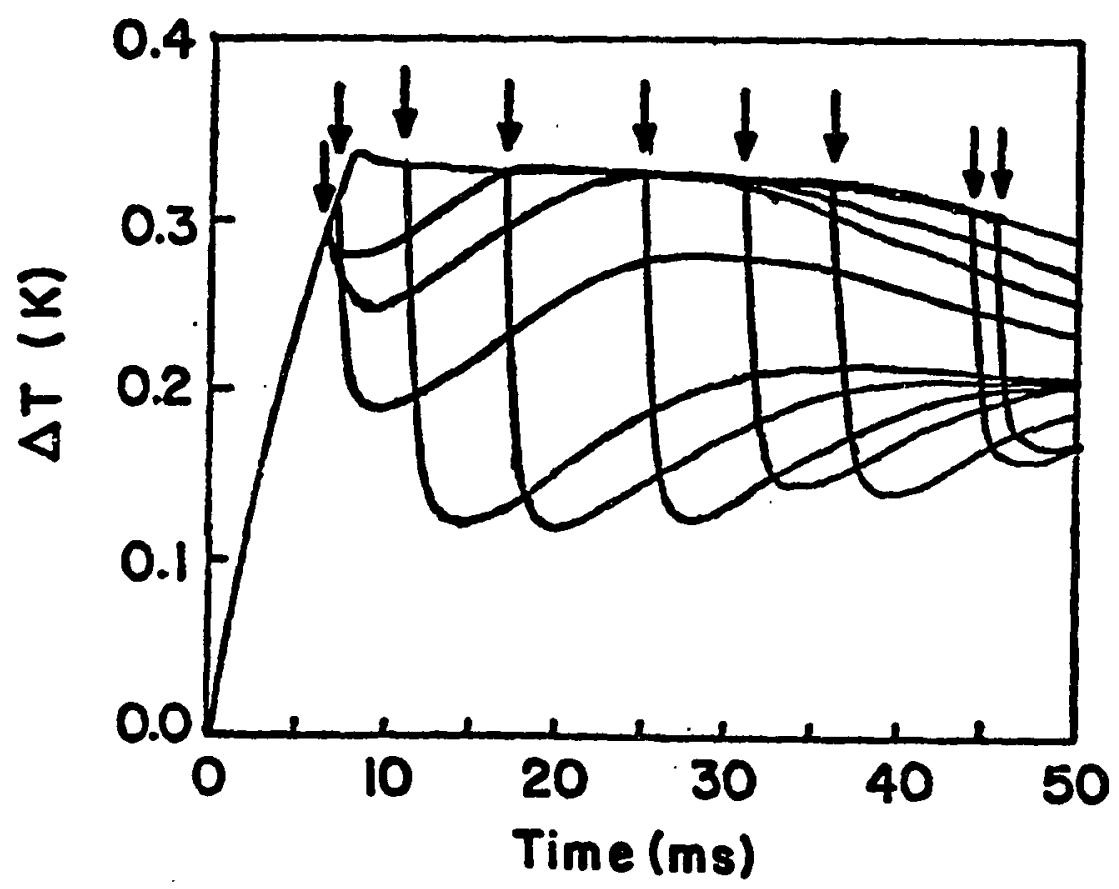

Figure 23. Composite record of ten $\Delta T$ vs. time curves. The light pulses are applied at successively earlier times. 
that there is no effect of a light pulse on the transient temperature curve if the pulse is applied before the superheat has reached a sufficiently high value. These observations suggest that the mechanism of heat transfer enhancement is not effective until the liquid helium superheat has reached a definite threshold value somewhat below the homogeneous nucleation temperature. That the threshold effect is a temperature threshold (and not a time threshold) is most clearly illustrated in Fig. 24 where the effect is shown for two slightly different heating rates for the solid. The threshold (denoted by the arrows) is found to occur at nearly the same superheat of about $0.3 \mathrm{~K}$ in both cases. This observation of a temperature threshold is further evidence that the light is indeed affecting the heat transfer between the bismuth crystal and liquid helium, rather than just triggering some artifact of the system such as photoconductivity or the photoelectromagnetic effect in bismuth (zitter 1965a, 1965b، Young 1960, Morimoto et al. 1977, Fukase and Nishina 1970). Figure 25 is a composite record of several experiments illustrating the effects of varying the intensity of the light pulse that is applied. Although the absolute light intensity could not be accurately measured with our current experimental apparatus, it is clear that a greater temperature drop is associated with higher light intensity. The light effect is pronounced enough so that it even occurs with ordinary room lighting. The result of allowing 


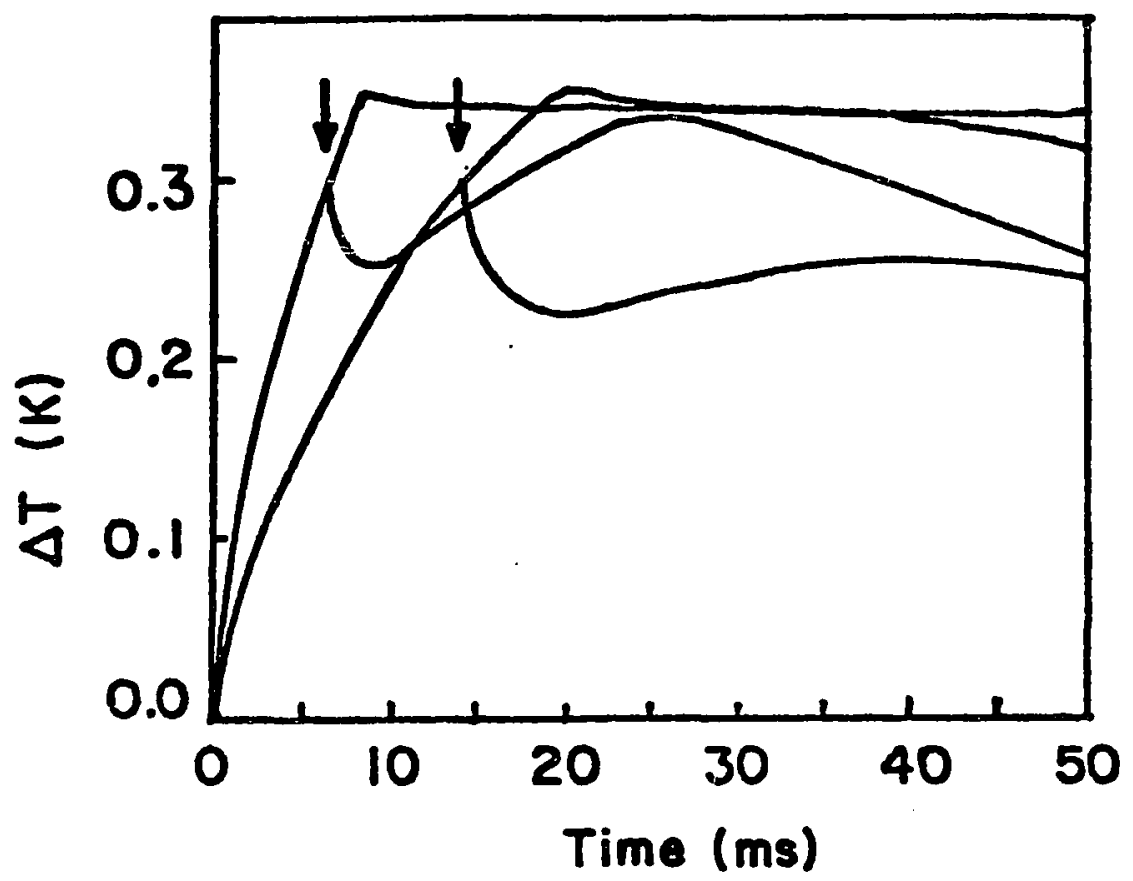

Figure 24. $\Delta \mathrm{T}$ vs. time curves at two different heating rates. The arrows indicate the threshold for the light effect. Note that at both heating rates, the threshold is at the same superheat temperature.

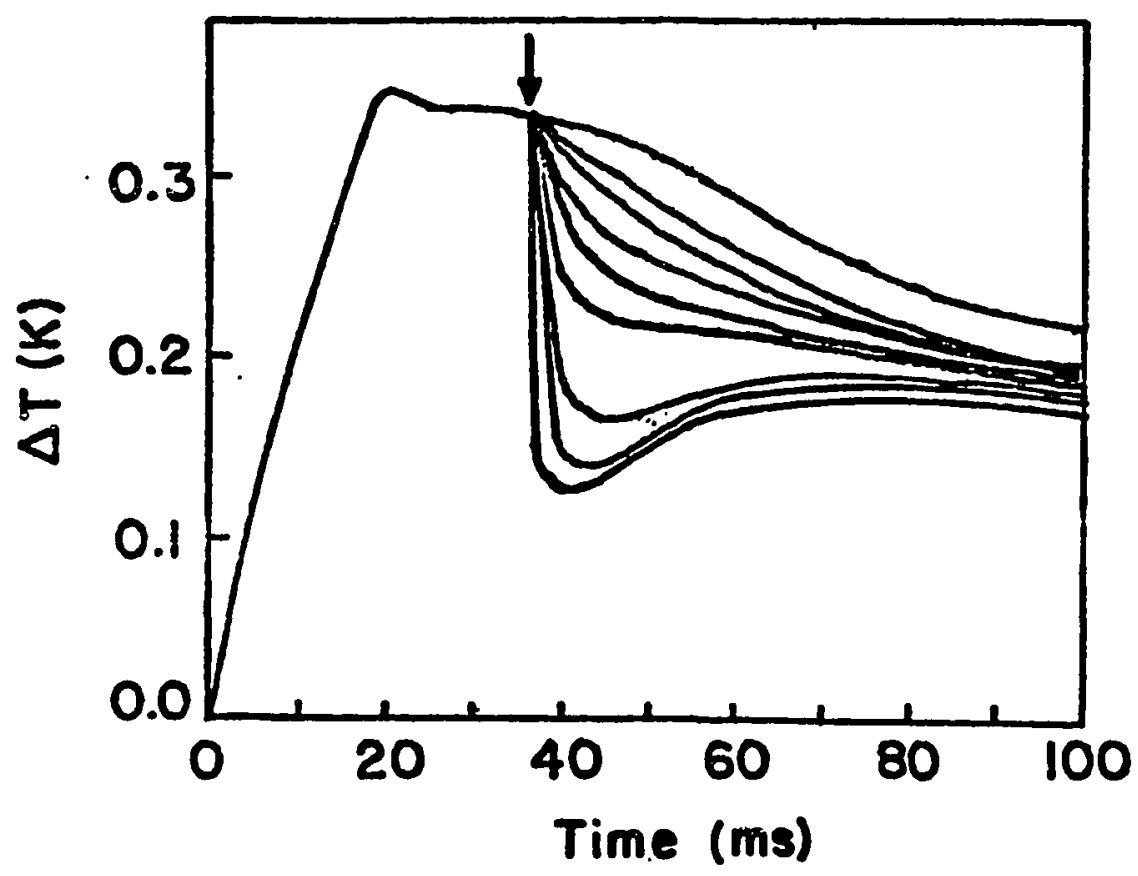

Figure 25. Effect of light pulse intensity on $\Delta T$ vs. time curve. Effect of eight different intensities are shown. The top most curve is the no light case and the lowest curve occured with the strongest intensity light. The intermediate cases are for progressively increasing relative intensities. 
the fluorescent room lights to shine through the unsilvered part of the glass Dewar into the bismuth-helium system during the entire duration of the temperature transient is shown in curve b of Fig. 26. For comparison, curve a shows the temperature versus time dependence for the case of no light falling on the system. The low intensity continuous room lighting has no effect on the initial part of the temperature rise, but accentuates the fall in temperature which follows the sharp temperature peak. However, if a very strong steady light source is used, curve $\underline{c}$ results. Above a certain superheat temperature, the transient superheat temperature deviates from the initial part of the temperature rise corresponding to the no light case.

In all cases in which the superheat temperature is above a certain threshold value the drop in the transient superheat temperature occurs upon application of the light pulse and the system subsequently returns to steady-state nucleate boiling. A light pulse induced momentary decrease in the superheat temperature is observed even in the case of steady-state nucleate boiling if the associated superheat temperature is above the threshold temperature value. It is also worth emphasizing the point that none of the light induced effects discussed in this section are observed if the Dewar is shielded from the light (for example by cloth or cardboard) when the strobe is flashed. Thus the results 


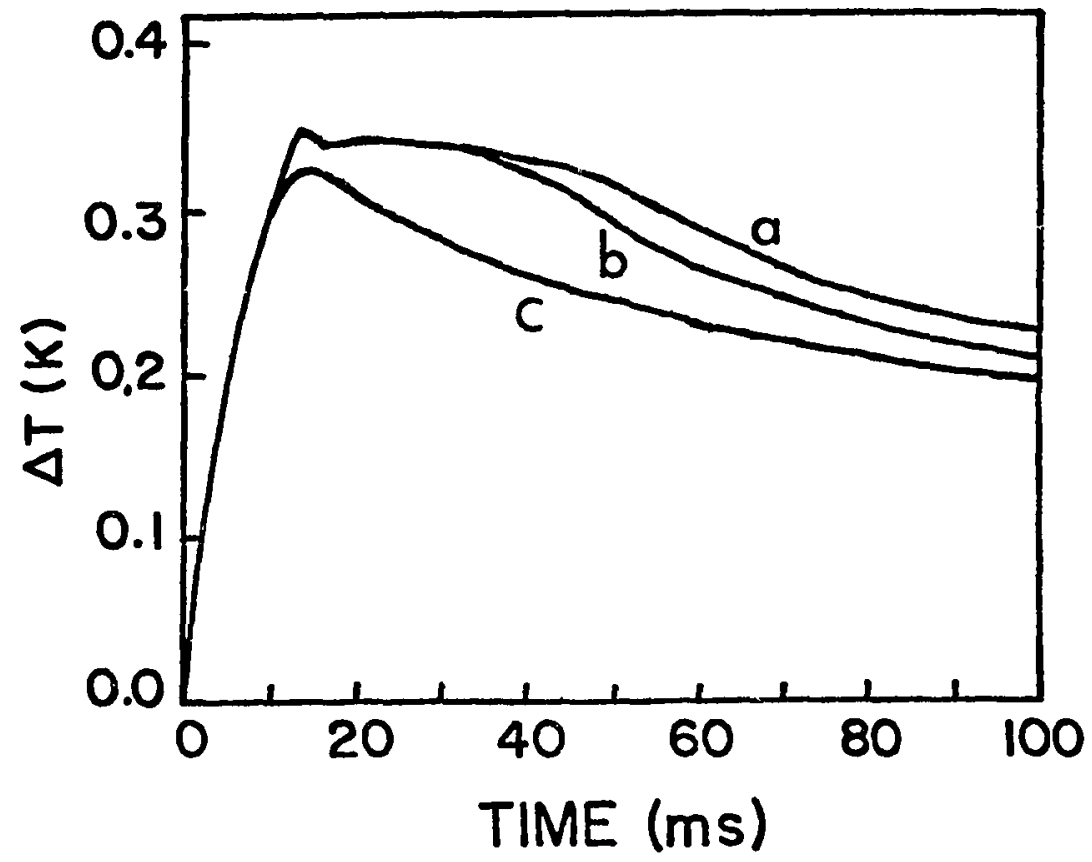

Figure 26. Effect of steady room light on the $\Delta T$ vs. time curve. Curve a is determined in complete darkness, curve $b$ is with fluorescent room light on, and curve $\underline{c}$ is with high intensity steady light. 
are not an artifact due, for example, to electrical pickup, but are exclusively the consequence of light falling on the system.

\section{DISCUSSION}

The most interesting feature of the light induced enhancement of transient heat transfer, as described in the previous section, is the existence of a temperature threshold $\Delta T_{\text {IT }}$ below which no effect due to a light pulse could be observed. We have found that the value of this temperature threshold does not depend on the size or geometry of the crystal used. This was determined by repeating the experiments with three different bismuth crystals of varied dimensions. The light effect was also studied at several magnetic fields ranging from $0.4 \mathrm{~T}$ to $2 \mathrm{~T}$ and at various orientations with respect to the crystal. No observable effect on the magnitude of $\Delta T_{I T}$ was found. The duration of the light pulse also does not appear to have any effect on the temperature threshold. The values of $\Delta T_{L T}$ as determined using two different pulse widths, $40 \mathrm{\mu s}$ and $3 \mathrm{~ms}$, were identical. There does not seem to be any influence of the surface conditions of the crystal on $\Delta T_{I T}$ either. An electropolished crystal and a crystal having numerous small etch pits on its surface produced identical results. A possible effect of the wavelength of the light pulse on the temperature threshold was also investigated. Two different colors were selected, 
red and blue. No significant change in $\Delta T_{L T}$ was observed. However, there was an indication that red light produced a slightly higher value of $\Delta \mathrm{T}_{L \mathrm{~T}}, 0.015 \mathrm{~K}$, than blue light. This result is somewhat uncertain since the intensities of the lights were widely different.

It was expected that the intensity of the light should have an effect on the temperature threshold. Further investigations indeed revealed that $\Delta \mathrm{T}_{\mathrm{LT}}$ does not have a sharply defined value but depends to an extent on the intensity of the light pulse and the heating rate of the crystal. Figure 27 shows the results of our study of the dependence of the light threshold $\Delta T_{L T}$ on the applied power per unit area of the crystal at three widely different light intensities indicated as Low, Medium, and High. Note that above an applied power value of roughly $40 \mathrm{mw} / \mathrm{cm}^{2}$, the threshold temperature quickly approaches a constant value for any given light intensity, and in particular the High intensity. Moreover, the effect of light intensity on $\Delta \mathrm{T}_{\mathrm{IT}}$ tends to saturate at higher intensities as indicated by the apparent overlap of the curves corresponding to Medium and High intensity light for power values above $40 \mathrm{~mW} / \mathrm{cm}^{2}$ as shown in Fig. 27. It is worthwhile to mention at this point that this power value of $40 \mathrm{~mW} / \mathrm{cm}^{2}$ roughly separates the rounded temperature overshoots from the overshoots with sharp peaks in the no-light case (see Chapter III). This can also be inferred from the nature of the dashed curve in Fig. 27 which represents the locus of the 


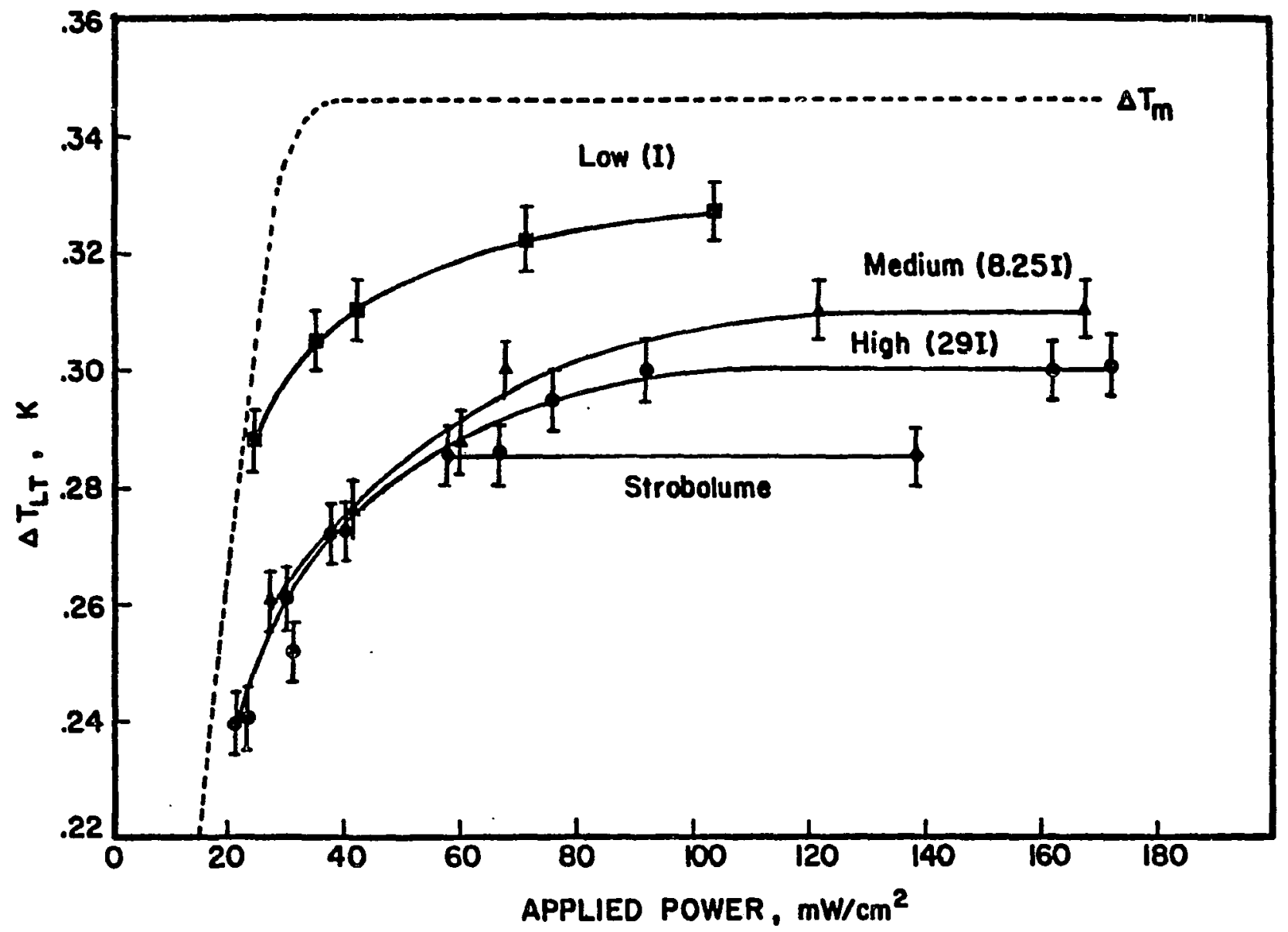

Figure 27. $\Delta T_{\text {IT }}$ as a function of applied power. The light threshold superheat $\Delta T_{\text {IT }}$ is shown as a function of the applied power for different light pulse intensities $I$. The intensity of the strobolume is significantly higher than the High intensity. The dashed curve represents the locus of the maximum superheat temperature of the overshoots as determined in the absence of light. 
maximum superheat temperature of the overshoots as determined in the absence of any light pulse. As mentioned earlier, the constant superheat temperature is a characteristic of the sharp peaks in the overshoots and corresponds to the homogeneous nucleation temperature of liquid helium. A distinguishing feature of the transient superheat curves associated with applied power values above $40 \mathrm{mw} / \mathrm{cm}^{2}$ is that in the initial stages up to the sharp peaks, the superheating of the liquid helium in contact with the bismuth crystal occurs primarily due to thermal conduction. On the other hand, in the case of the rounded overshoots, appreciable heterogeneous nucleation takes place on the surface of the crystal before the maximum superheat is reached. The fact we wish to point out in this connection is that $\Delta T_{L T}$ is not significantly affected by heating rate as long as the light threshold is determined for applied power values corresponding to situations in which the bismuth-helium interface is not subjected to additional complications due to heterogeneous nucleation, i.e. above $40 \mathrm{~mW} / \mathrm{cm}^{2}$. The trend of the Medium and High intensity curves in Fig. 27 suggests that an extrapolation of the behavior of the temperature threshold at still higher intensities may be possible. It seems reasonable to assume that for significantly higher intensity light pulse the temperature threshold will perhaps saturate to a relatively more sharply defined value somewhere in between $0.28 \mathrm{~K}-$ $0.29 \mathrm{~K}$ independent of the applied power, at least above 
$40 \mathrm{mw} / \mathrm{cm}^{2}$. Limited data with the strobolume, which has a significantly higher intensity light pulse than the Strobotac, supports such an assumption. Thus, one may identify a rather definite threshold temperature value as a characteristic parameter of the bismuth-helium system. at any given helium bath temperature.

No definite explanation of the light effect could be established on the basis of the existing data. However, several hypotheses have been consicered and are presented below.

Photoelectron bubble hypothesis

The sudden enhancement of heat transfer is most likely caused by the rapid nucleation of bubbles in the superheated liquid. There are certain physical reasons for expecting a possible light induced enhancement of transient heat transfer to occur in liquid helium. It has been well established that free electrons exist in liquid helium for only a few picoseconds before becoming surrounded by a helium-free spherical cavity which is generally referred to as an electron bubble (Ferrel 1951, Sommer 1964, Woolf and Rayfield 1965, Kuper 1961, Jortner et al. 1965, Fowler and Dexter 1968, Onn and Silver 1971). The potential for the formation of electron bubbles in liquid helium suggests the possibility of a two-step process in which (1) electrons are detached from the bismuth surface by the action of light and (2) electron bubbles are then formed which serve as 
nuclei for ordinary vapor bubbles which subsequently grow rapidly in the superheated liquid by extracting energy from the thermal boundary layer and the bismuth surface.

support for the electron bubble hypothesis can be found in the work of Akulichev and Boguslavskii (1972) which showed that the cavitation strength of liquid helium is reduced by the presence of electron bubbles. In the case of superheated helium I, the presence of electron bubbles should similarly reduce the temperature at which superheated liquid helium spontaneously nucleates. Thus, after a pulse of light stimulates the creation of electron bubbles, the helium should homogeneously nucleate at a superheat $\Delta \mathrm{T}_{\mathrm{h}}^{\prime}=\Delta \mathrm{T}_{\mathrm{h}}-\delta \mathrm{T}$, where $\Delta \mathrm{T}_{\mathrm{h}}$ is the homogeneous nucleation superheat temperature in the absence of electron bubbles and $\delta T$ is the temperature reduction caused by the formation of electron bubbles immediately after a light pulse is applied.

Further support for the electron bubble hypothesis comes from the suggestion of Hernandez $(1970,1973)$ that electrons have a strong tendency to form electron bubbles within regions of spatially localized density fluctuations. Since we presumably increase density fluctuations by superheating the liquid helium, the formation of electron bubbles around the ejected photoelectrons should be enhanced.

Figure 28 shows how the homogeneous nucleation temperature $T_{h}$ is affected by the presence of electron 


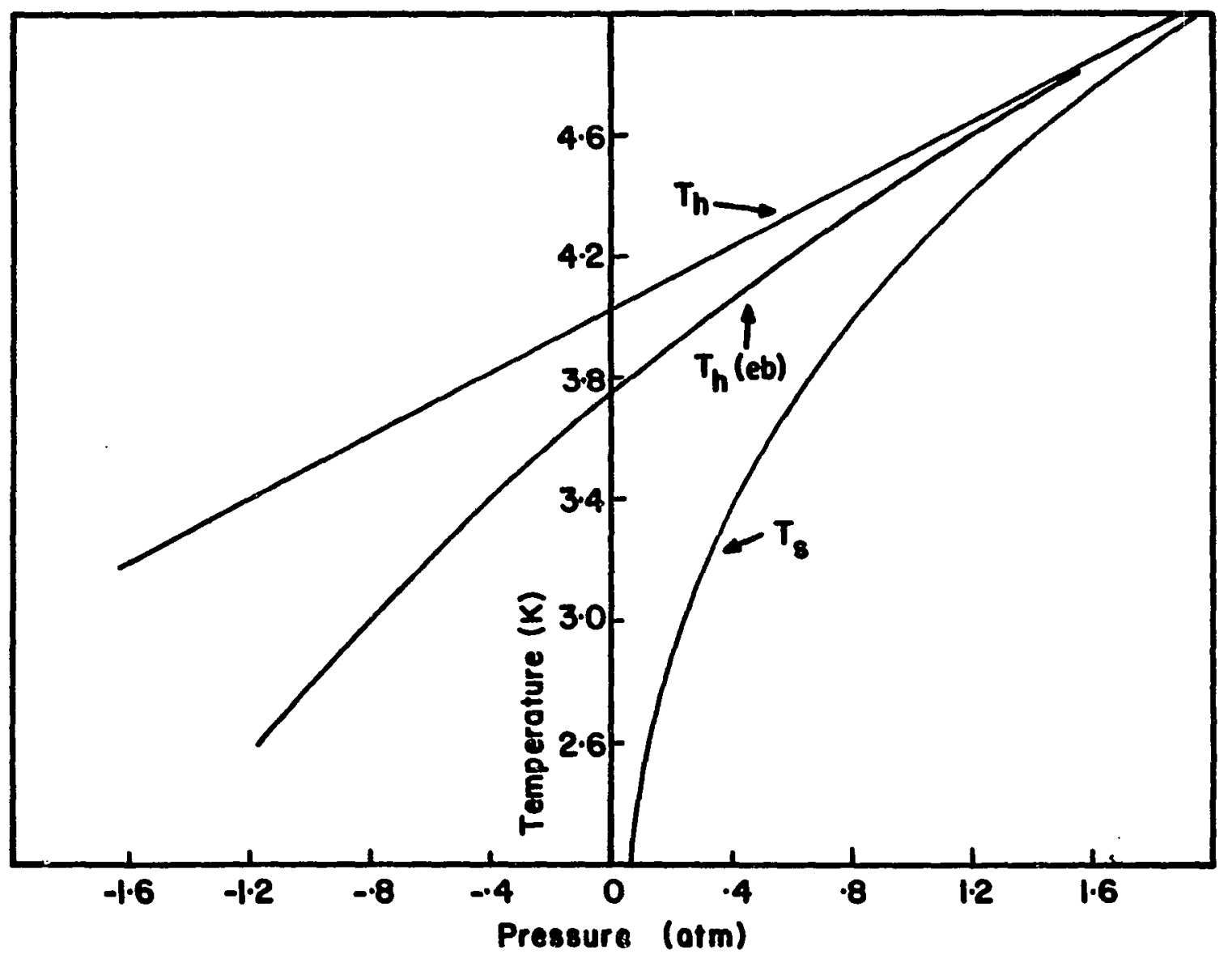

Figure 28. Homogeneous nucleation temperature $T$ as a function of pressure. The curve $T_{h}(e b)$ represents the nucleation temperature as affected by the presence of electron bubbles in the liquid. $T_{s}$ is the saturated helium bath temperature. 
bubbles. The curve $T_{h}(e b)$ was determined following Akulichev and Boguslavskii (1972) who derive an expression for the cavitation strength of liquid helium in the presence of electron bubbles. In Fig. 29 we compare our experimental data of $\Delta \mathrm{T}_{\mathrm{LT}}$ with the superheat corresponding to the homogeneous nucleation temperature of liquid helium as affected by electron bubbles, $\Delta T_{h}(e b)=T_{h}(e b)-T_{S^{\prime}}$ determined from Fig. 28 for a wide range of saturation bath temperatures $\mathbf{T}_{\mathbf{S}}$. The dashed curve in Fig. 29 represents the homogeneous nucleation superheat in the absence of electron bubble. The agreement between theory and experiment is good. However, it does not constitute conclusive evidence in favor of the electron bubble hypothesis. The main uncertainty stems from the indefiniteness in the determination of the temperature threshold. Until adequate information regarding the exact nature of the effect of wavelength, light intensity, and heating power on the temperature threshold is available nothing definite about the validity of the photoelectron bubble hypothesis can be said.

In order to make some of these ideas clearer, there are two points concerning the photoemission of electrons from the bismuth surface and the effect of wavelength that should be discussed. At liquid helium temperatures the mean free path of the electrons are long compared to the classical skin depth. In this situation, the electrons which are photoemitted are thought to be contributed 


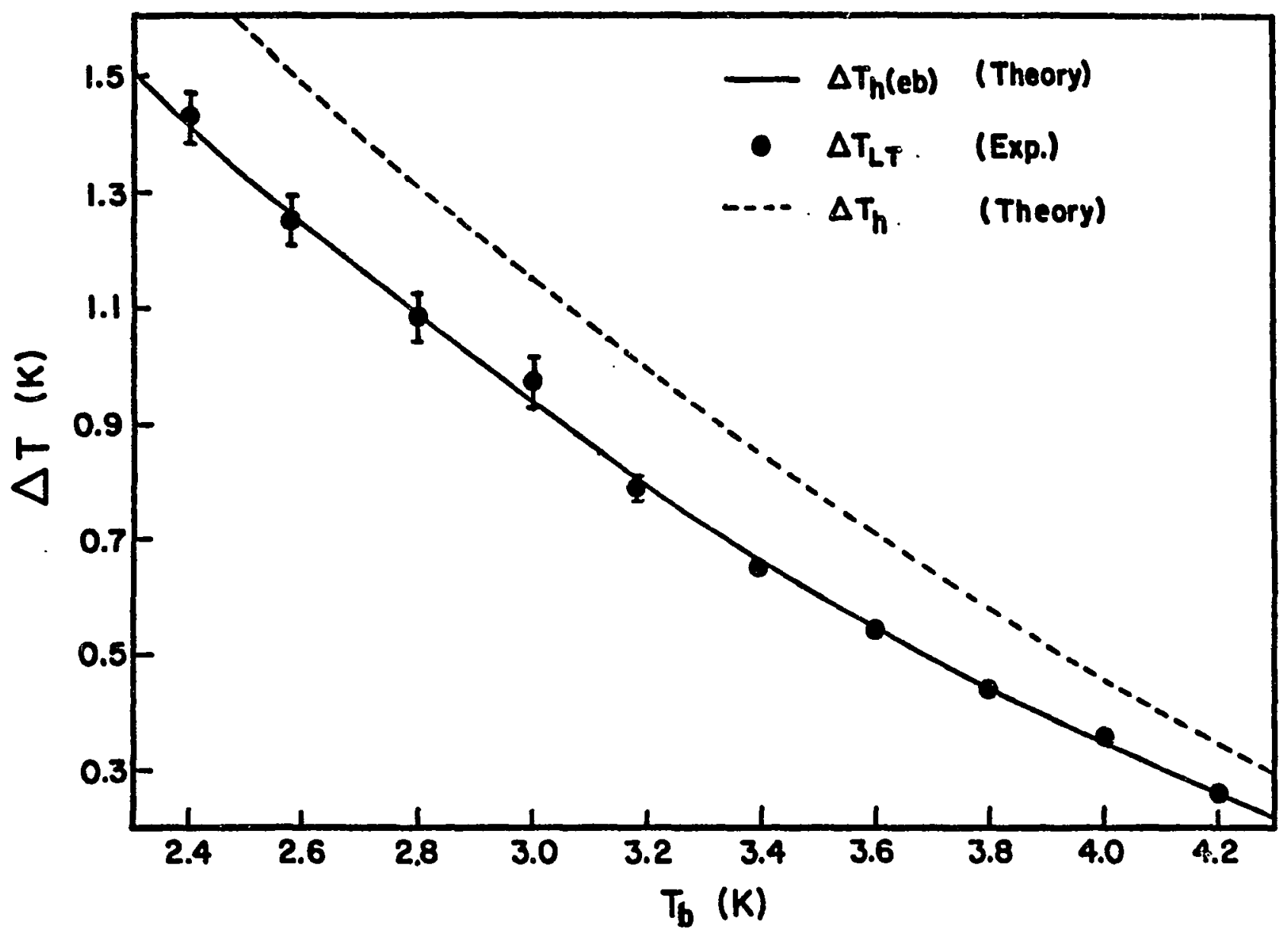

Figure 29. $\Delta \mathrm{T}_{\mathrm{T}}$ as a function bath temperature. Experimental data (dark circles) are compared with theory (solid line). The dashed line represents the homogeneous nucleation superheat without the effects of electron bubbles. 
primarily from the surface states. As regard to the effect of wavelength, the enhancement of heat transfer is observed with visible light. We even see the effect with red light. One might expect that the work function related to photoemission corresponds to a wavelength $\lambda_{c}$ (for bismuth $\lambda_{c}=3075 \AA$ (Haas 1972)) which is far enough into the ultraviolet so as to preclude any emission of electron from the bismuth surface, since very little ultraviolet light reaches the bismuth surface in one glass Dewar system. However, if visible light is used, Smejtek (1975) has suggested that light of wavelength $\lambda>\lambda_{c}$ could produce photoelectrons which could exist in liquid helium near the bismuth surface for a time long enough for electron bubbles to form. The idea can best be illustrated schematically by the simple energy diagram in Fig. $30 . \Phi$ is the work function of the solid-liquid helium interface and $A B$ represents the energy of a photon of wavelength $\lambda>\lambda_{c}$. If an electron near the Fermi level absorbs such a photon, it will have an energy corresponding to point $B$, and it can therefore move outside the surface into the liquid helium if its velocity is normal to the surface. It is therefore energetically allowed for an electron to penetrate into the liquid helium as far as point $C$ which is on the image potential curve. Although most such electrons would probably not penetrate into the liquid as far as $c$ due to deflection by elastic collisions with helium atoms, they are likely to still remain in the liquid long enough to 


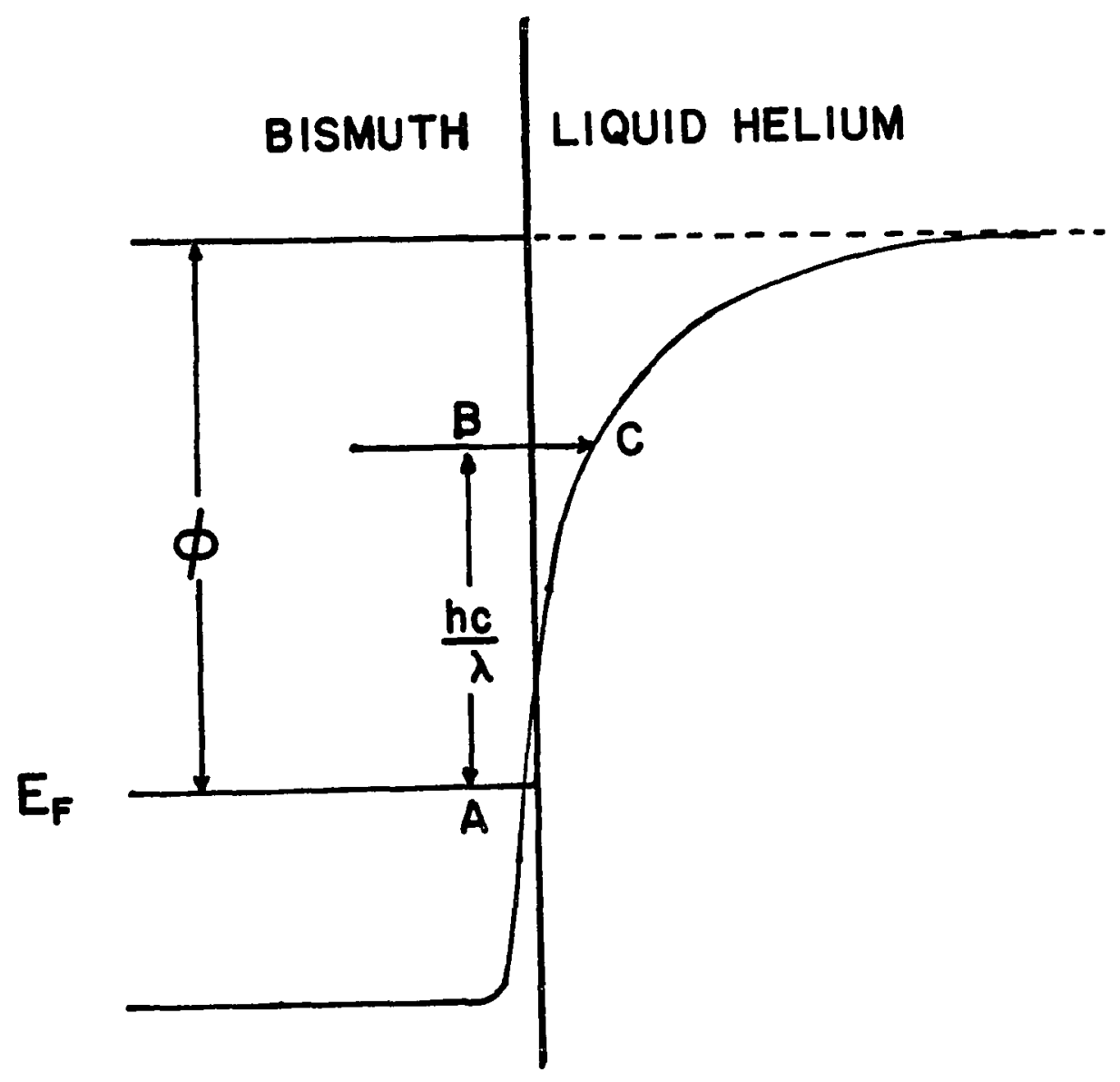

Figure 30. Energy level diagram for an electron in the vicinity of the bismuth-helium interface. $E_{F}$ represents the Fermi level energy of the semi-metal bismuth and the curve represents the potential energy of an electron inside and outside the bismuth surface. The photoemitted electrons from the bismuth surface are energetically allowed to penetrate into the liquid as far as point $C$ which is on the image potential curve. 
form electron bubbles. This picture suggests that it is quite possible for electrons photoemitted by light of wavelength $\lambda>\lambda_{c}$ to form electron bubbles before being drawn back into the bismuth surface by the image force. However, in order to obtain more conclusive evidence in favor of the electron bubble hypothesis an additional experiment was designed. It was expected that if electron bubbles were generated by some independent means without using light and injected into the superheated liquid at an appropriate time, then effects similar to those observed with the light pulse could be reproduced. Professor Russel Donnelly of the University of Oregon suggested that an $\mathrm{Am}^{241}$ radioactive source be used since it is a strong a-emitter and in liquid helium, due to ionization of the helium atoms, it should generate a copious supply of electrons which in turn would produce a large number of electron bubbles. These electron bubbles then could be electrically gated and injected into the superheated helium layer in contact with the bismuth crystal in a controlled manner. The experimental arrangement is shown in Fig. 31. The a-particles are stopped in liquid helium within a distance less than $0.3 \mathrm{~mm}$. So the electron bubbles are expected to be produced in the region between the grid, which is a fine wire mesh, and the source. Due to the positive polarity of the grid with respect to the source plate, the electron bubbles are normally collected on to the grid and positive ions on to the source plate. If by using 


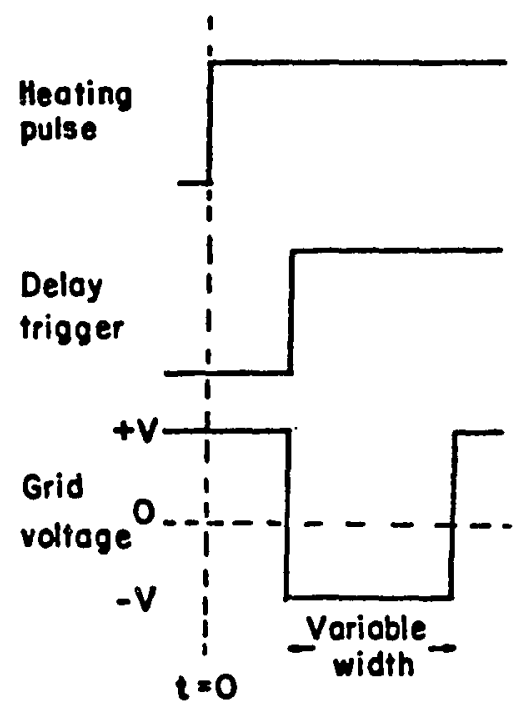

Bismuth
crystol

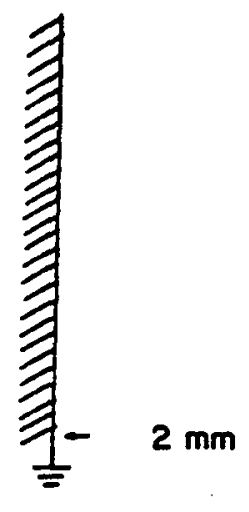

Grid Source

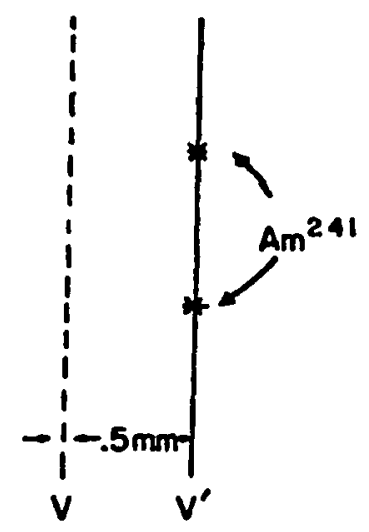

$V($ Max. $)=150 \mathrm{~V}$

$v-v^{\prime}=30 v$

Figure 31. Schematic representation of the experimental set up. The stars indicate the two $\mathrm{Am}^{241}$ radiactive sources. The relative timing of the heating pulse, delay trigger, and the grid voltage pulses are also shown.

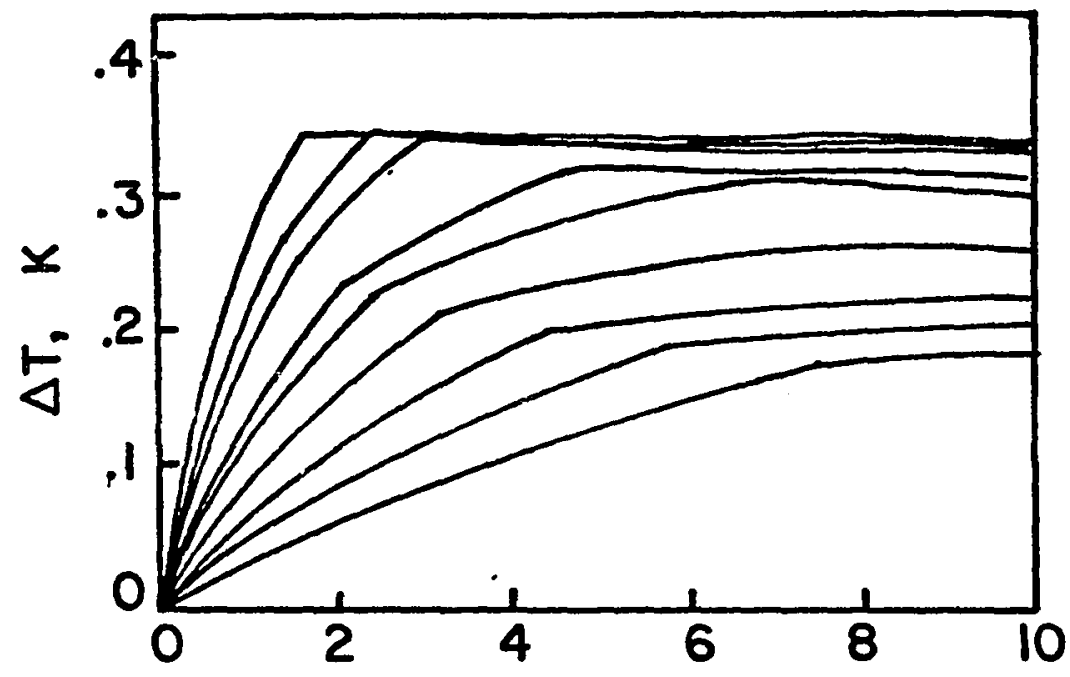

TIME, ms

Figure 32. Superheat temperature $\Delta T$ vs. time. Sharp changes in the heating rates are observed in the presence of the radiactive source. The bath temperature is $4.2 \mathrm{~K}$. 
a delayed trigger signal the voltage on the grid is momentarily lowered from $+V$ to $-V$, then the electron bubbles should be repelled by the source plate and grid and be attracted by the bismuth crystal which is always at ground potential. By this mechanism injection of electron bubbles into the region between the crystal and the grid could be achieved. Unfortunately, the results of this experiment were totally inconclusive in regards to showing any effects of the electron bubbles. A typical set of superheat temperature vs. time curves for several values of applied powers is shown in Fig. 32. Note the sudden sharp change in the heating rates of the crystal during the initial period of increasing superheat. The superheat at which the slope change occurs varies with the applied power. However, if the overshoots are below a superheat temperature value of roughly $0.2 \mathrm{~K}$, the effect of slope change is not observed. Surprisingly, at first, this observed effect was completely independent of the electronic gating procedure described above. A range of voltage combinations, duration of the negative voltage and delay times were tried without producing any observable effect in the transient superheat curves. A lack of any influence of the voltages suggested that the anticipated effect due to electron bubbles must be insignificantly small compared to some extraneous sources which might be causing the observed behavior in the $\Delta T$ vs. time curves. The observed effect 
as shown in Fig. 32 was also found to be independent of the orientation and the magnitude of the external magnetic field.

It was later recognized that in addition to the $\alpha$-particles the $\mathrm{Am}^{241}$ radioactive source also produced $64 \mathrm{keV} \mathrm{x}$-rays. These X-rays interacted either directly with the superheated helium or indirectly through the ejection of photoelectrons from the bismuth surface and initiated nucleation which caused the sudden change in the transient heat transfer as observed. Initiation of nucleation in a superheated liquid by $\gamma$-photons and high energy electrons ( $\delta$-electrons) have been studied by Skripov (1974) and several other investigators in the past. See Aleksandrov et al. (1963) for a review.

It is quite evident now that an altogether different approach is needed to produce electron bubbles. The most promising method appears to be the field emission of electrons from a metal-insulator-metal system, first developed by onn et al. (1974) for producing electron bubbles in several cryogenic liquids. In the absence of support from such studies the evidence for the photoelectron bubble hypothesis must remain inconclusive.

Phonon hypothesis

A second possibility is that a burst of phonons is generated in the bismuth crystal by the light flash, and that the energy of the phonons is coupled into the liquid 
helium to form bubble nuclei which then grow rapidly in the superheated liquid. From the high Kapitza conductance value of bismuth (see Chapter II) one can expect that the phonon transmission coefficient of the bismuth-helium interface is relatively large. This suggests that phonons can easily enter the superheated liquid helium in contact with the crystal surface. It is worth mentioning in this connection that recently Kavetskaya et al. (1979) reported the excitation of acoustic pulses in liquid helium by optical pumping of germanium. A rough approximation of the phonon energy based on the Debye temperature of bismuth ( $117 \mathrm{~K}$ ) shows that it is comparable to the energy required to form critical nuclei in liquid helium $76 \mathrm{kT}$, where $\mathrm{k}$ is the Boltzmann constant and $\mathrm{T}$ is the helium bath temperature. A major fraction of this $76 \mathrm{kT}$ is, however, supplied by the superheated liquid and a relatively small amount of additional energy is needed to homogeneously nucleate the liquid.

Recently, Yonemitsu (1979) has proposed new mechanisms by which phonons or supersonics can be generated in bismuth. He suggests that the light energy absorbed by the electrons (a non-local effect) near the surface of the crystal is carried deeper inside and released. This should lead to a shear stress between the interior of the crystal and the surface and result in the generation of supersonics. On the other hand, if the absorption is local, phonons are excited by collisions and the energy is carried away by 
phonons without generating supersonics. These proposed mechanisms have also been experimentally verified (Yonemitsu 1979). However, in order to quantitatively explain the temperature threshold on the basis of the phonon for supersonics) hypothesis, information regarding the nature of the phonon-helium (or supersonics-helium) interaction is needed. At the present time details of such mechanisms are not at all obvious.

\section{SIGNIFICANCE}

The light induced enhancement of transient heat transfer offers the exciting possibility that it could lead to methods of suppressing undesirable transient temperature excursions in the cryostatic stabilization of superconducting devices which are refrigerated in liquid helium. The methods might involve light or electron injection, e.g., using a $\beta$ - or $\alpha$-emitter. It is worth pointing out the possible practical ease with which cryostatic stabilization might be carried out; even a low intensity visible light source such as an ordinary incandescent lamp is observed to affect transient temperature excursions.

On a more fundamental level, the basic question of the effect of light on the transient heat transfer processes in a solid-liquid helium system needs to be studied. The discovery of the light effect phenomenon opens the door to an examination of the effect of light on the initiation of nucleate boiling and may also lead to another tool for 
investigations of electron bubbles in liquid helium. Furthermore, evidence that light affects the initiation of nucleate boiling has important implications for studies of nucleation rates and bubble growth processes which employ high speed photography and high intensity lighting as an experimental method, and also for any nucleation experiments in which glass Dewars or windows are used. 


\title{
CHAPTER VII
}

\author{
PREMATURE TRANSITION TO STABLE FILM BOILING INITIATED \\ BY POWER TRANSIENTS IN IIQUID NITROGEN
}

\section{INTRODUCTION}

During the past several decades, extensive work has been carried out on the problem of steady-state pool boiling. In particular, in studies in which the heat flux is controlled, it is well known that within a certain range of heat flux values, the solid-liquid system can exist in either of two stable pool boiling steady-states corresponding to the usual nucleate and film boiling processes. For a given heat flux within this range, either of these two stable conditions can be attained depending upon the manner in which the boiling process is initiated.

In typical steady-state experiments in which the heat flux through the solid-liquid interface is controlled, changes in the heat $f l u x$ are initiated in a quasi-steadystate manner, i.e., slowly enough and in small enough increments that the system is always maintained at, or near, a steady-state. Under these conditions, as the heat flux is increased, the system undergoes the well-known transition from convective heat transfer to nucleate boiling and can sustain the latter process until a critical steady-state 
peak heat flux, $q_{s}^{p}$, is attained above which a transition to film boiling occurs. In contrast to this quasi-steadystate manner of initiating boiling, we examine a process of rapid initiation in which the system may momentarily be far from a steady-state. More specifically, we have investigated the question: if the power to the solid is applied suddenly, can a transition to stable film boiling be initiated in a bath of Iiquid nitrogen at power levels substantially lower than that corresponding to the steadystate peak heat flux?

The fact that such a "premature" transition to film boiling may be initiated in non-cryogenic liquids under certain special circumstances has been previously reported by several workers. In a study primarily concerned with steady-state measurements in water, Gaertner (1965) noted that film boiling would often occur prematurely when the furnance power input was increased suddenly. However, several other investigators (Tolubinskiy et al. 1975, Borishanskiy and Fokin 1969, Lurie and Johnson 1962) who have carried out transient heating experiments in water observed no premature transition to film boiling. Roemer (1969) reported premature film boiling as a result of a power transient in unaged cylinarical test elements immersed in water, but not with test samples previously aged by boiling in water. Tolubinskiy et al. (1976) reported that under transient conditions such a transition does occur for aged samples in ethanol and acetone. 
In the case of cryogenic liquids, the possibility of a premature transition to stable film boiling initiated by a power transient apparently remains an open question that has not received much attention. If such a premature transition does exist, it may impose a significant limitation in the design of cryogenic devices which are subjected to transients during operation or switch-on in situations in which film boiling cannot be tolerated.

our investigations have shown that such a premature transition to stable film boiling does indeed occur for thin wires in liquid nitrogen. We have initiated such transitions at atmospheric pressure in response to stepfunction inputs of power into moderately thin test wires of platinum, copper, and constantan. For the cases examined, a transition to stable film boiling was observed for power input values as low as approximately 40 per cent of the power values corresponding to the steady-state peak heat flux. We have also examined the transient heat flux through the surface of the wires into liquid nitrogen and find that the premature transition to stable film boiling can occur even though the observed maximum values attained by the transient heat flux may lie substantially below the steady-state peak heat flux. We find that all these results hold for wire test samples in liquid nitrogen regardless of whether the wires were unaged or aged by prior sustained boiling at a high heat flux. 
Finally, we suggest that premature transitions are allowed by the incomplete development of the convective forces of bubble detachment which would normally act to stabilize the nucleate boiling process. The weakness of such forces of detachment immediately after the initiation of step-function heating allows for the coalescence of bubbles and for the consequent formation of a stable vapor film at the surface of the test wire at power levels considerably lower than those associated with the steadystate peak heat flux. In view of this picture, in the Results and Discussion section we describe a demonstration of the inhibition of premature transitions by a type of externally induced forced convection around the test wire.

\section{EXPERIMENTAL}

The experimental system consists of a test section of thin heating wire mounted vertically in a pool of liquid nitrogen which is contained in a $5 \mathrm{~cm}$ i.d. pyrex Dewar. The heating wire is $10 \mathrm{~cm}$ long and two potential leads are attached $2.5 \mathrm{~cm}$ from each end of the wire. Platinum $(0.102 \mathrm{~mm}$ diameter), copper $(0.127 \mathrm{~mm})$, and constantan $(0.127 \mathrm{~mm}, 0.254 \mathrm{~mm})$ have been used as heating wire materials. Platinum and copper, in particular, were chosen because of their large temperature coefficient of resistance and high degree of linearity in resistivity vs. temperature over a wide range of temperatures. 
For the purpose of thermally isolating the wire-liquid nitrogen system, the Dewar containing the test section is in turn nested within another Dewar which is filled with liquid nitrogen up to a level slightly higher than the bath in the inner Dewar. Prior to any experimentation, the inner Dewar is first cleaned with acetone and then flushed with liquid nitrogen, and a nitrogen atmosphere is maintained inside the Dewar before transferring liquid nitrogen into it. With this procedure it is possible to suppress unwanted bubbling from the surfaces of the inner Dewar and the test section, and thus to maintain the liquid nitrogen bath in a nearly quiescent state. All data were taken at atmospheric pressure.

Measurement of superheat temperature

We employ a conventional hot wire technique in which a metal wire is heated electrically and its superheat temperature $\Delta T(t)$ is determined as a function of time $t$ by measuring the resulting change in its electrical resistance $\Delta R(t)$. Fig. 33 shows a schematic diagram of the circuitry employed. A step-pulse from a pulse generator is fed to the series circuit consisting of the heating wire $\left(R_{1}\right)$ and two sets of $0.25 \Omega, 100$ Watt resistors, $R_{2}$ and $R_{3}$, of low temperature coefficient of resistance. The current I through the circuit is determined by monitoring the voltage across the resistor $R_{3}$. The voltages $R_{1} I$ and $R_{2} I$ after amplification by the instrumentation amplifiers 


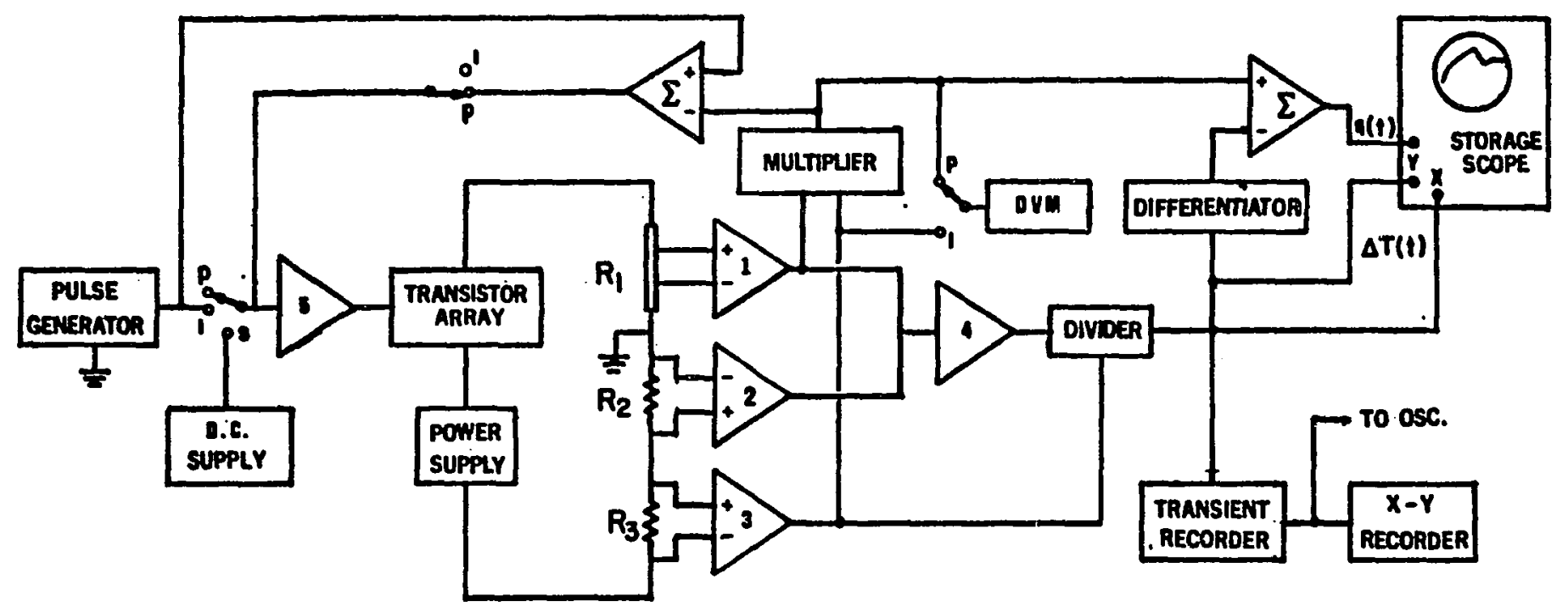

Figure 33. Schematic circuit diagram. 
1 and 2 are fed to amplifier 4 to obtain the voltage difference $\Delta \mathrm{V}$. These amplifiers have a gain bandwidth product of $40 \mathrm{MHz}$. If $\mathrm{A}_{1}$ and $\mathrm{A}_{2}$ are the amplification factors (typically $\sim 2$ ) of the amplifiers 1 and 2 we have for the duration of the pulse

$$
\begin{aligned}
\Delta V & =A_{1} R_{1}(t) I-A_{2} R_{2} I \\
& =A_{1}\left[R_{1}(0)+\Delta R(t)\right] I-A_{2} R_{2} I,
\end{aligned}
$$

where $R_{1}(0)$ is the resistance of the heating wire at the bath temperature. Initially, the amplification factor $\mathrm{A}_{2}$ is adjusted such that $A_{1} R_{1}(0) I=A_{2} R_{2} I$. Thus, we have $\Delta V=A_{1} \Delta R(t) I$ for the duration of the pulse. A divider circuit is used to divide this voltage difference $\Delta V$ by the current signal from amplifier 3 to obtain a time-varying signal which is proportional to $\Delta R(t)$. Since $\Delta R(t)$ is very nearly directly proportional to $\Delta T(t)$, the amplification of the divider can be adjusted so that its output reads the superheat temperature $\Delta T(t)$ directly over a restricted temperature range. This temperature signal can either be fed directly to a storage oscilloscope or stored in a digital sampling type transient recorder for subsequent display on an oscilloscope or an $x-y$ recorder. For a more accurate determination of temperature, reference is made to a calibration curve obtained from previous measurements of the test wire resistance as a function of known 
temperatures. When it is desired to display the entire range of superheat temperatures, including the film boiling temperature, a logarithmic amplifier is used for data compression.

An electronic timer is used to trigger the pulse generator at equal intervals of time. A sufficient time interval, typically at least 2 minutes, is set between trigger pulses to assure the reproducibility of the $\Delta T(t)$ curves. Before each set of measurements the test wire is cleaned with acetone.

The measured average bulk temperature of a test wire should be nearly equal to the wire's surface temperature because the response time $\tau\left(\tau=r^{2} / D\right.$, where $r$ and $D$ are the radius and the thermal diffusivity of the wire, respectively) of the wires used is small, typically of the order of $10 \mathrm{\mu s}$. The estimated overall uncertainty in the determination of the superheat temperature in these experiments is $0.5 \mathrm{~K}$.

Measurement of applied power to the wire and heat flux into the liguid

Since the terminology employed in the literature has sometimes been confusing, it is worth pointing out that in this work we make a distinction between the energy release rate into the wire, which we call the applied power per unit area, $p$, and the transient heat flux through the surface of the wire into the liquid, $q(t)$. The power $p$, 
where $p=I^{2}(t) R_{1}(t) /$ surface area, is turned on as a steppulse at time $t=0$ and is thereafter held at a constant value (except for the case, discussed below, in which we apply a constant current step). On the other hand the transient heat $f l u x$ is a time-varying quantity defined through the power balance equation:

$$
p=q(t)+C(T) \frac{d T}{d t}
$$

where $C(T)$ is the temperature-dependent heat capacity per unit area of the wire. Therefore, in transient heating experiments a distinction must be made between $p$ and $q(t)$. Note however that, as the time approaches infinity, the transient heat flux $q(t)$ asymptotically approaches its steady-state value, $\mathrm{q}_{\mathbf{s}}$, which equals the applied power: $q(t) \rightarrow q_{s}=p$ as $t+\infty$. In the next section it will often be convenient to resort to making comparisons between $q_{s}$ and $\mathrm{p}$. In particular, the power necessary to cause a premature transition to film boiling in the transient case will be compared with $q_{s}^{p}$, the heat flux value at which a transition to film boiling occurs in the steady-state case. The circuitry employed in this work is designed in such a way that the electronic system can be operated in two modes: constant power or constant current. In the constant power mode, the power is turned on at time $t=0$ as a step and maintained at a constant value for the duration or the applied step-pulse by employing an 
electronic feedback system which is described below. In the constant current mode, however, the current is kept constant after it is turned on as a step. Unless it is stated otherwise, our measurements were carried out in the constant power mode.

When the system is operated in the constant power mode, the feedback system operates as follows. The voltages $R_{1}(t) I(t)$ and $R_{3} I(t)$ are amplified and fed to a multiplier circuit which produces an output proportional to the power delivered to the part of the heating wire between the potential leads. This multiplier output is used to regulate the current delivered by amplifier 5 in order to maintain the power at a constant value for the duration of the steppulse. The response time of the feedback circuit has been experimentally determined to be $\sim 1 \mathrm{MHz}$. The system can also be operated in the constant current mode simply by breaking the feedback loop and feeding the output of the pulse generator directly to amplifier 5. A peak reading digital voltmeter is used to monitor either the current or the power when the system is used in either the constant current or the constant power mode, respectively. The uncertainty in the measurement of power is less than 28. The transient heat flux is determined by an analog method employing a modified operational amplifier differentiation circuit (Tobey et al. 1971) and a difference amplifier. We have found that this method of determining the heat flux is more accurate and convenient than the 
usual graphical differentiation method. The heat flux is obtained by taking the time-derivative of the $\Delta T(t)$ signal using the differentiation circuit and subtracting this timederivative from the multiplier output, $p$, by means of the difference amplifier in accordance with Eq. (7.1). To calibrate this heat flux circuit, the amplitude of the differentiated signal is adjusted to obtain zero heat flux output from the difference amplifier at time $t=0$. The frequency range of reliable operation of the differentiation circuit was determined by using a variable frequency triangular wave generator. The cut-off frequency of the circuit has been found to be approximately $5 \mathrm{kHz}$. Thus the response time characterizing the heat flux measurements is of the order of $0.2 \mathrm{~ms}$. A dual trace storage oscilloscope is used to simultaneously record both the transient heat flux $q(t)$ and the superheat temperature $\Delta T(t)$. Alternatively, by feeding the $\Delta T(t)$ signal to the $x$-input of the storage scope, $\mathrm{g}(t)$ can be displayed directly as a function of $\Delta T(t)$.

In connection with the determination of the heat flux, it should also be noted that the second term in Eq. (7.1) contains the wire's temperature-dependent heat capacity per unit area. Since liquid nitrogen temperatures are substantially lower than the Debye temperature of the materials employed for the test wires, the heat capacities of the test wires vary significantly with temperature during the course of a transient measurement. The magnitudes of 
the transient heat fluxes (shown in Figs. 35 and 36 and reported in the results section) have been corrected for this change in the heat capacity with temperature using Eq. (7.1).

In addition to the above transient measurements, we have also made conventional steady-state superheat vis. heat flux measurements which were carried out with the same system used in the transient case except that a d.c. voltage source was used instead of the pulse generator.

\section{RESULTS AND DISCUSSION}

For the case in which a constant power step is applied at time zero, typical transient superheat temperature $\Delta T(t)$, curves are shown in Fig. 34 for several values of the applied power per unit area. At low power values the superheat temperature monotonically attains a convective steady-state value as exhibited by curves $\underline{a}$ and $\underline{b}$. At higher power values $\Delta \mathrm{T}$ initially rises above, i.e., "overshoots," its steady-state nucleate boiling temperature as seen in curve c. As the power is still further increased, the rounded overshoots become progressively greater in magnitude and occur at increasingly earlier times (curve d). The overshoots in the transient temperature are attributed to a time lag in the onset of heterogeneous nucleation at the surface of the wire. Similar features in the transient temperature curves have been observed in helium (Brodie et al. 1977, Steward 1978), nitrogen (Steward 


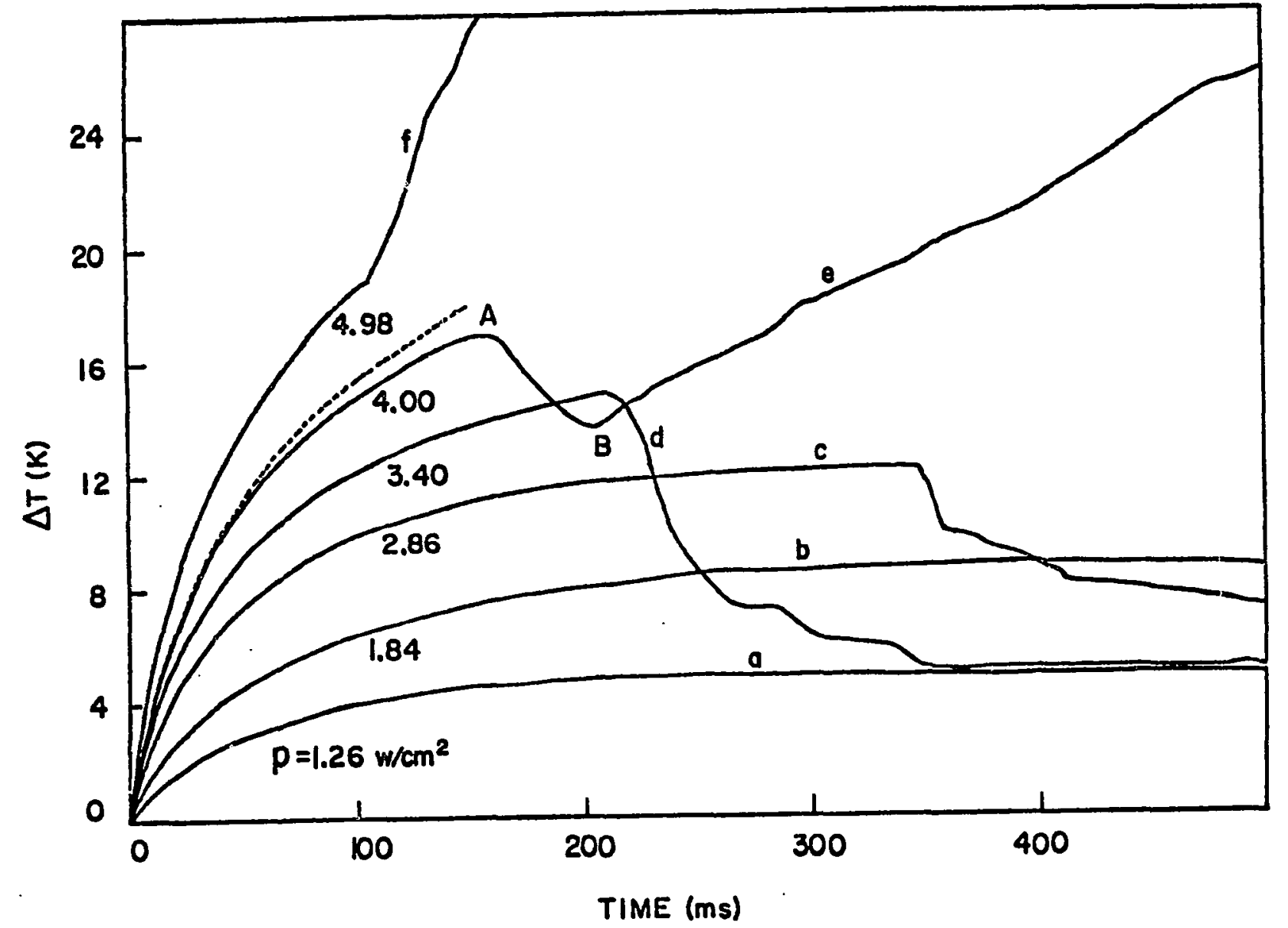

Figure 34. Superheat temperature $\Delta T$ vs. time. The temperature response as a function of time is show for the platinum wire with step-function applied power input initiated at time $t=0$. The bath temperature is $77.35 \mathrm{~K}$. Thenumber associated with each curve is the applied power per unit area of the wire surface in $\mathrm{W} / \mathrm{cm}^{2}$. The dashed line represents the temperature rise prediction for curve $e$ under the assumption of pure heat conduction between the wire and the liquid for a uniform uniform constant rate of heat generation in the wire. 
1978), and also in non-cryogenic liquids such as water (Tolubinskiy et al. 1976, Johnson 1970) and several organic liquids (Tolubinskiy et al. 1976).

The characteristics of the $\Delta T(t)$ curves at several applied power values serve as the basis of our study of the critical power $p_{c r}$, the power value at and above which a transition takes place to stable film boiling. Note in particular curve e of Fig. 34. After an initial increase to point $A$, the temperature of the wire momentarily decreases to point $B$ and then increases again until stable film boiling is established. For a power level even slightly below the $4.00 \mathrm{~W} / \mathrm{cm}^{2}$ associated with curve e, the system always relaxes to a stable nucleate boiling steadystate after an initial overshoot in temperature, whereas for higher power values the system always undergoes a transition to steady-state film boiling, as demonstrated by curve $\underline{\underline{f}}$. Thus the power value associated with curve e is the critical power, $\mathrm{p}_{\mathrm{cr}}$. The critical power values for the platinum, copper, and constantan heating wires were determined and are presented in Table III. The relative magnitudes of $p_{c r}$ and $q_{s}^{p}$ are also known as a ratio, $p_{c r} / q_{s}$. This ratio is found to be 0.38 for the platinum heating wire. For the copper and constantan heating wires the ratio $\mathrm{p}_{\mathrm{cr}} / \mathrm{q}_{\mathrm{S}}^{\mathrm{p}}$ is found to be between 0.42 and 0.47 . Thus, for wires of all three materials, we find that a premature transition to stable steady-state film boiling can be 
initiated by step-function power imputs of the order of one-half of the steady-state peak heat flux.

TABLE III

THE CRITICAL POWER, STEADY STATE PEAK HEAT

FLUX, AND THE MINIMUM VALUE OF THE

PEAK TRANSIENT HEAT FLUX

\begin{tabular}{lcccccc}
\hline Test wire & Diameter & $\mathrm{p}_{\mathrm{cr}}$ & $\mathrm{q}_{\mathrm{s}}^{\mathrm{p}}$ & $\mathrm{q}_{\mathrm{tm}}^{\mathrm{p}}$ & $\frac{\mathrm{p}_{\mathrm{cr}}}{\mathrm{q}_{\mathrm{s}}^{\mathrm{p}}}$ & $\frac{\mathrm{q}_{\mathrm{tm}}^{\mathrm{p}}}{\mathrm{q}_{\mathrm{S}}^{\mathrm{p}}}$ \\
Material & $\mathrm{mm}$ & $\mathrm{W} / \mathrm{cm}^{2}$ & $\mathrm{w} / \mathrm{cm}^{2}$ & $\mathrm{w} / \mathrm{cm}^{2}$ & & \\
\hline Platinum & 0.102 & 4.00 & 10.5 & 4.5 & 0.38 & 0.43 \\
Copper & 0.127 & 4.25 & 10.2 & 4.1 & 0.42 & 0.40 \\
Constantan & 0.127 & 5.56 & 13.2 & 5.4 & 0.42 & 0.41 \\
Constantan & 0.254 & 6.47 & 13.8 & 6.6 & 0.47 & 0.48 \\
\hline
\end{tabular}

Several additional comments concerning the transient temperature curves are worth making at this point. First, as discussed previously, we ensured the consistent reproducibility of the critical power data by allowing a sufficient relaxation time for the system (typically at least 2 minutes) between subsequent step-pulses. Second, since it was reported by Roemer (1969) for the case of water that aging increased the critical power value, we have examined the question of aging by continuously heating the test wire in liquid nitrogen for an hour at a moderately high heat flux (typically 50 per cent of $q_{s}^{p}$ ). However, we found 
that such prior aging of the wire sample did not have a significant effect on $p_{C r}$. It is also worth pointing out in connection with Fig. 34 that the initial stage of the transient temperature rise is well explained by the assumption that the heat transport mechanism between the wire and the liquid is purely thermal conduction. This is illustrated, for example, by the agreement between the experimental curve and the dashed line associated with curve e of Fig. 34. The dashed line represents the temperature rise predicted by assuming that the heat transfer in the liquid is purely due to thermal conduction. The heat conduction expression employed is for the case of cylindrical geometry and for a constant rate of heat generation in the bulk of the wire (Appendix C).

In addition to the determination of $\mathrm{p}_{\mathrm{Cr}}$, another question of special interest concerns the behavior of the transient heat flux from the wire into the liquid during the course of a premature transition to film boiling. In particular, we are interested in determining whether there is a critical value of the transient heat flux (analogous to the steady-state peak heat flux $q_{S}^{p}$ ), which, if exceeded, always results in a transition to film boiling. Consequently, the heat flux $q(t)$ and the corresponding superheat temperature $\Delta T(t)$ were simultaneously determined, using Eq. (1) as discussed in the experimental section. The measurements were carried out over a range of applied power values up to a maximum value equal to $q_{s}^{p}$. 
Figure 35 shows the behavior of the transient superheat temperature and the corresponding transient heat flux as a function of time for three applied power values. In case (a) it can be seen that although the heat flux momentarily exceeds $q_{s}^{p}$, the system relaxes to steady-state nucleate boiling. In case (b) on the other hand, even though the peak in the transient heat flux is lower than $\mathrm{q}_{\mathrm{S}}^{\mathrm{p}}$, the system finally attains a film boiling steady-state. The behavior of the heat flux typified by case (b) is associated with applied power values which are restricted to a narrow range of values immediately above $\mathrm{p}_{\mathrm{cr}}$. In case (c), the heat flux rises smoothly to a peak value $q_{t}^{p}$ which is substantially lower than $q_{s}^{p}$. Again, the system finally attains a film boiling steady-state. The behavior typified by case (c) is observed for applied power values ranging from slightly above $p_{C r}$ up to the highest power used. It is interesting to determine how small a peak value of the transient heat flux can be for the case in which there is a transition to film boiling. These values of $q_{t m}^{p}$ were obtained by applying heating step-pulses of different power values and by determining the peak transient heat flux $q_{t}^{p}$ at each power. The minimum value of $q_{t}^{p}$ for which a transition occurs to steady-state film boiling is denoted by $q_{t m}^{p}$ and is shown in Table Ir.

We therefore find that the momentary attainment of a heat flux value equal to or exceeding the steady-state peak heat flux does not necessarily cause a transition to 

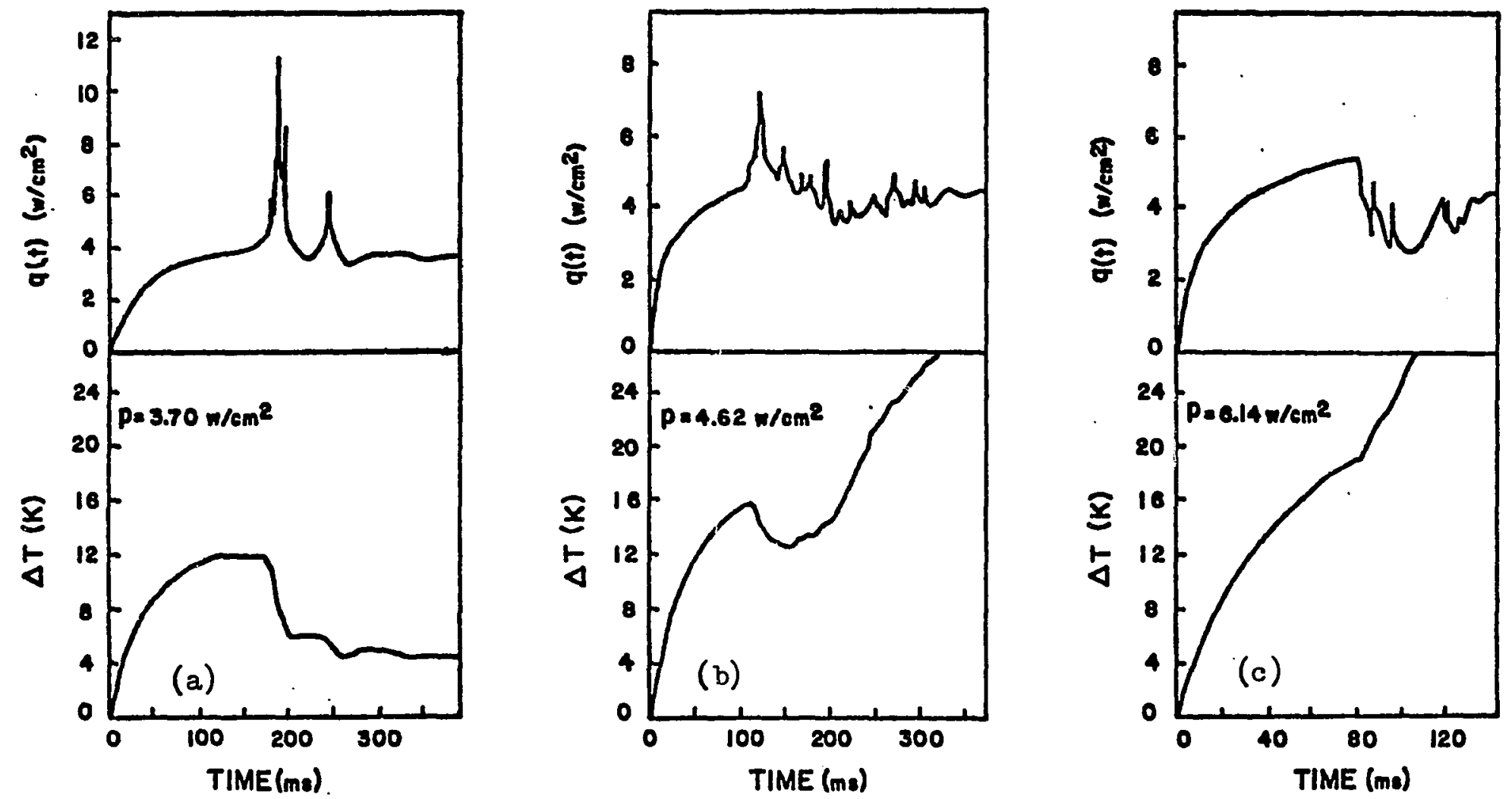

Figure 35. Heat flux $q$ and superheat temperature $\Delta T$ vs. time. The superheat temperature and the corresponding heat flux are show for three applied power values as a function of time for the platinum wire immersed in a bath of saturated liquid nitrogen at $77.35 \mathrm{~K}$. The transient heat flux should be compared against a steady-state peak heat flux of $10.5 \mathrm{~W} / \mathrm{cm}^{2}$ for this test wire. 
stable film boiling. Moreover, a transition to stable film boiling can take place even though the transient heat flux may never attain the steady-state peak heat flux value. In fact, as determined from the $q_{t m}^{p}$ values in Table III a transition to stáble film boiling may occur even for cases in which the peak value of the transient heat flux is only about 40 per cent of $\mathrm{q}_{\mathrm{s}}^{\mathrm{P}}$. We conclude that a particular critical value of the transient heat flux which by itself provides a criterion for the onset of a premature transition does not exist for this sytem.

The transient behavior which is typified by Fig. 34 can also be viewed in the heat flux - temperature phase plane. In Fig. 36 several trajectories of the transient heat flux vs. superheat temperature are shown. Trajectories d', $\underline{e}^{\prime}$, and $\underline{f}^{\prime}$ in Fig. 36 correspond respectively to the temperature vs. time curves $\underline{\underline{d}}, \underline{e}$, and $\underline{\underline{f}}$ for Fig. 34 . Our steady-state heat flux vs. superheat temperature measurements are also shown for the purpose of comparison with the transient results. Trajectory $\underline{d}^{\prime}$ represents the behavior of the transient heat transfer process where $\Delta T$ initially overshoots the final steady-state nucleate boiling temperatures The characteristic loop in trajectory $\underline{e}^{\prime}$ of Fig. 36 is associated with the type of temperature overshoot shown by curve e in Fig. 34. Again, trajectories $e^{\prime}$ and f' graphically show the premature transition to film boiling. Note that in both cases the peak in the transient heat flux remains considerably below the steady-state peak 


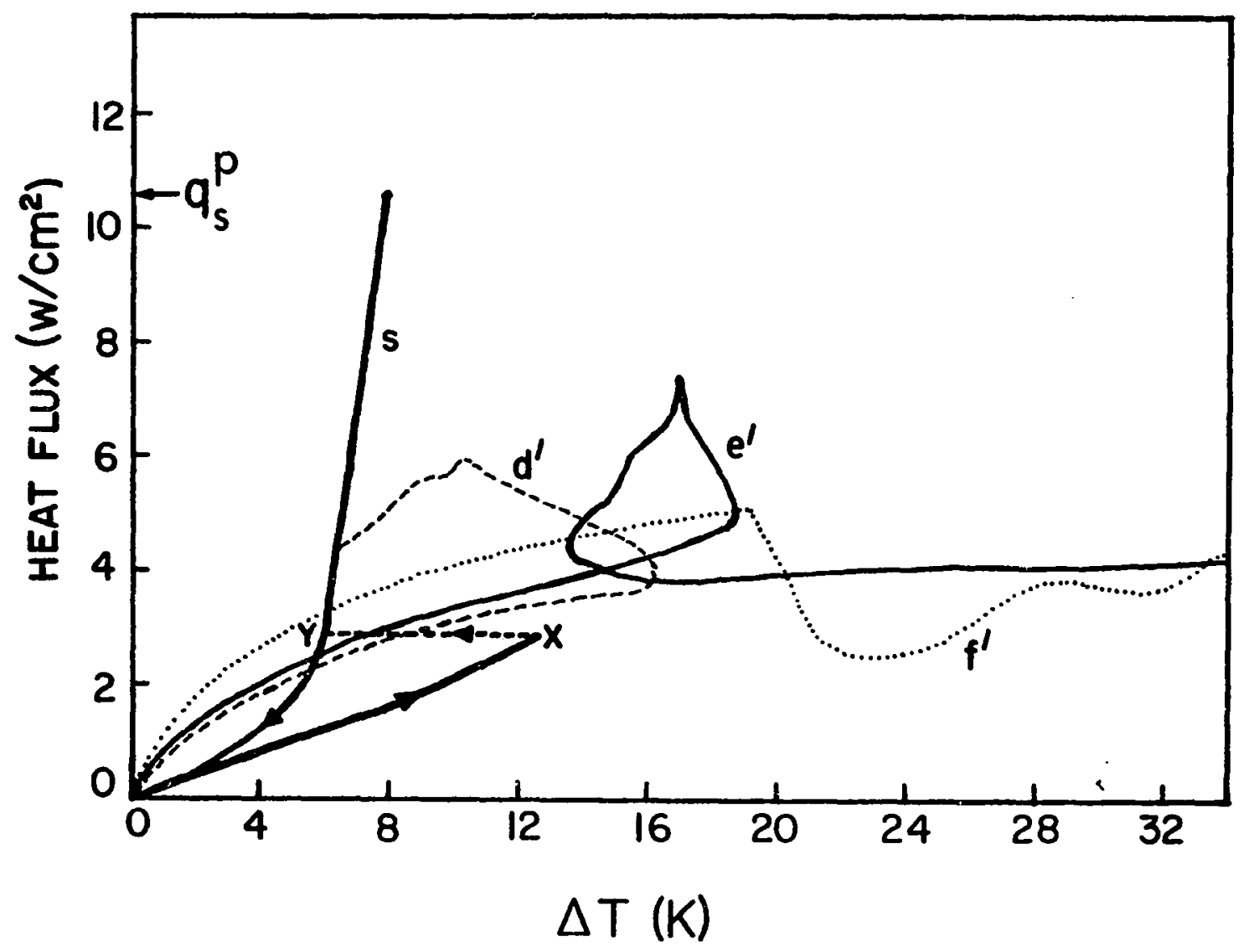

Figure 36. Heat flux q vs. superheat temperature $\Delta T$. Transient (curves $\underline{d}^{\prime}, \underline{e}^{\prime}$, and $f^{\prime}$ ) and steady-state (curve $s$ ) heat fluxes aje shown as a function of superheat temperature for the platinum wire in liquid nitrogen at $77.4 \mathrm{~K}$. The abrupt $x \rightarrow y$ transition between convective and nucleate boiling in the steady-state curve occurs for experiments in which the heat flux is slowly increased. 
heat flux. It is also worth noting in connection with Fig. 36 that a knowledge of only the usual steady-state variables $q$ and $\Delta T$ at some initial instant of time is in general not sufficient to predict the future course of the transient process. This fact can be inferred from the existence of the loop in curve $\underline{e}^{\prime}, \mathrm{Fig} .36$. The $q, \Delta \mathrm{T}$ trajectory intersects itself at the bottom of the loop. Since two different paths exist through the point of intersection a knowledge of $q$ and $T$ at the point of the intersection is insufficient to uniquely specify the future path of the system.

In addition to the above studies in which the power applied to the heating wire is maintained at a constant value for the duration of the applied step-pulse, we have also investigated the case in which the heating current is kept constant. We find qualitatively similar behavior in the characteristics of the transient heating curves in the two cases, although certain details in the fine structure of the curves may vary. In the constant current case the applied power to the heating wire, $I^{2} R$, varies with time in proportion to the change in the temperature-dependent electrical resistance of the wire. We therefore do not seek to identify a critical power value associated with the premature transition to stable film boiling. However, as in the constant power case, the peak transient heat flux $q_{t}^{p}$ can also be considerably lower than $q_{s}^{p}$. For example, the average value of the ratio $q_{t m}^{p} / q_{s}^{p}$ in the constant 
current case has been found to be approximately 0.5 for the heating wires used.

We suggest that the premature transition to stable film boiling, initiated by a step-function input of power, is primarily due to a time lag in the full development of strong turbulent convective forces in the liquid in the vicinity of the active nucleation sites at the wire surface. When nucleate boiling is initiated in a quasi-steady-state manner by slowly increasing the heat flux, turbulent convective forces are generated in the liquid adjacent to the wire surface as a consequence of the bubble growth and detachment process. The development of these convective forces acts to effectively stabilize the nucleate boiling process by further promoting subsequent bubble detachment and by consequently inhibiting the coalescence of bubbles into a stable vapor film at the surface of the test wire. A transition to stable film boiling occurs at the steadystate peak heat flux for which the existing fully developed bubble detachment forces can no longer compete against the high rate of vapor generation to prevent coalescence of bubbles. In contrast to the quasi-steady-state case just described, when nucleation is initiated suddenly by a stepfunction input of power, bubble growth takes place in the absence of fully developed convective forces. In this latter case, the ultimate final stable state of the system is determined by the relative strength of two processes: (1) the rapid onset of heterogeneous nucleation and 
subsequent bubble growth, and (2) development of turbulent convective forces in the liquid. The first process acts to create a vapor film at the surface of the wire through coalescence of bubbles, whereas the latter process acts to inhibit coalescence. At moderate power inputs, the coalescence of bubbles on the surface of the wire may not be sufficient to compete against the convective forces generated in their neighborhood and consequently a stable nucleate boiling state is attained. However, at and above the critical power value $\mathrm{p}_{\mathrm{cr}}$, the rate of vapor generation is such that bubbles coalesce and form a stable vapor film around the wire surface before the turbulent convective forces have had the time to become developed enough to prevent stable film formation. This explanation of the premature transition to film boiling in nitrogen due to the sudden initiation of heating is qualitatively similar to those put forth for the case of non-cryogenic liquids by Borishanskiy and Fokin (1969) and by Tokarev (1976) . The preceding explanation also suggests that externally induced forces which aid in bubble detachment and inhibit coalescence at the wire surface should act to stabilize the nucleate boiling process and to inhibit a premature transition to stable film boiling. We have carried out a simple demonstration which is consistent with this explanation. A flat electric heater $(2.5 \mathrm{~cm} \times 2 \mathrm{~cm})$, immersed in the nitrogen bath $3 \mathrm{~cm}$ directly below the lower end of the platinum test wire, was used to generate 
bubbles. These bubbles rose upward through the liquid and produced a form of externally-induced turbulent convection around the heating wire. The step-function heating experiment was performed with the test wire immersed in this convective environment and the critical power value $p_{C r}$ was determined. While such an experiment does not provide the more conventional direct determination of the magnitude of the forced convective flow around the wire, the power to the external heater does yield at least a qualitative measure of the degree of convection since the induced flow around the wire can be controlled by the heater power. The results are shown in Fig. 37, where the critical power value $\mathrm{p}_{\mathrm{Cr}}$ is plotted as a function of the heater power. The critical power value is found to increase with increasing heater power. The result is consistent with the picture that turbulent convection around the wire acts to stabilize the nucleate boiling process by inhibiting the formation of a stable gaseous film around the wire after step-function heating is initiated. A somewhat similar forced convective experiment was carried out by Borishanskiy and Fokin (1969) with similar results. It is also worth emphasizing that methods can be devised by which the effect of the premature transition to film boiling can be suppressed or inhibited in situations where it cannot be tolerated. Further experiments along these lines may be of potential practical importance. 


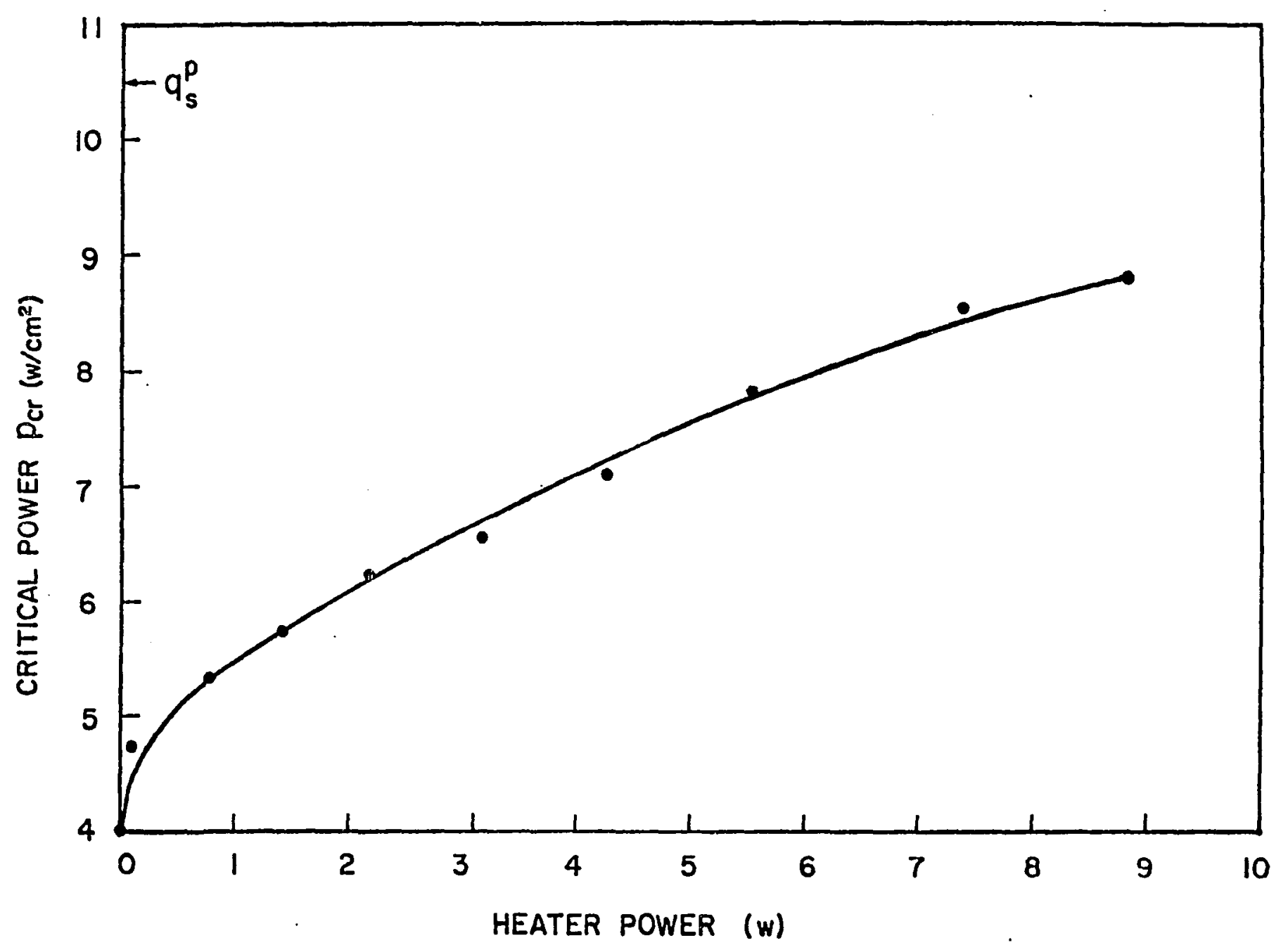

Figure 37. Critical power $p_{\text {r }}$ vs, heater power. The critical power at which a premature transition to film boiling očcurs is shown as a function of the power applied to a heater below the test wire. 
Finally, it should again be emphasized that the results presented here were obtained for thin vertical wires. There are indications in several organic liquids (Tolubinskiy et al. 1975, Borishanskiy and Fokin 1969, Tolubinskiy et al. 1977a, Tolubinskiy et al. 1977b) that the size of the heating test element may make a difference in the ease with which a premature transition is induced. 


\section{REFERENCES}

Abraham, F. F. 1974. Homogeneous nucleation theory. Academic Press, New York.

Akulichev, V. A. and Boguslavskii, Yu. Ya. 1972. Sov. Phys. - JETP. 35. 1012 .

Aleksandrov, Yu. A., Voronov, G. S., Gorbunkov, V. M., Delone, N. B., and Nechayev, Yu. I. 1967. Bubble chambers, Indiana University Press, Bloomington.

Atkins, K. R. and Narahara, Y. 1965. Phys. Rev. 138, A437.

Baidakov, V. G., Skripov, V. P., and Kaverin, A. M. 1975. SOv. Phys. - JETP. 40, 335 .

Bald, W. B. 1973. Cryogenics. 13, 457.

Beams, J. W. 1959. Phys. Fluids. 2, 1.

Becker, R. and Doring, w. 1935. Ann. Phys. 24, 719 and 752 .

Bewilogua, I., Knoner, R., and Vinzelberg, H. 1975. Cryogenics. 15,121 .

Bhagat, S. M. and Manchon, Jr., D. D. 1967 . Phys. Rev. 164,966 .

Binder, K. and Stauffer, D. 1976. Adv. Phys. 25, 343.

Blander, M. 1979. Adv. Colloid and Interface Sci. 10, 1.

Blander, M., Hengstenberg, D., and Katz, J. I. 1971.

J. Phys. Chem. 75, 3613.

Blander, M. and Katz, J. I. 1973. Fourteenth national heat transfer conference, Atlanta, Georgia.

Blander, M. and Katz, J. L. 1975. AIChE Journal. 21, 853.

Bocherts, R. H. 1975. Cryogenetics. 15, 385.

Borishanskiy, V. M. and Fokin, B. S. 1969. Heat Transfer Sov. Res. $1,27$. 
Bradley, R. H. and Witte, L. C. 1972. Nuc. Sci. Eng. $\underline{48}$, 387.

Brauer, F. E., Green, N. W., and Mesler, R. B. 1968. Nucl. Sci. Eng. 31, 551 .

Brodie, L. C., Sinha, D. N., Semura, J. S., and Sanford, C. E. 1977. J. Appl. Phys. 48, 2882 .

Brodie, L. C., Sinha, D. N., Sanford, C. E., and Semura, J. S. 1978. 99th ASME Winter annual meeting, San Francisco. 78-WA/HT-4.

Bugg, D. V. 1959. Prog. Nucl. Phys. ‥ 1.

Burgess, D. S., Murphy, J. N., and Zabetakis, M. G. 1970. U. S. Bureau of Mines report to U. S. Coast Guard.

Byk, A. 1921. Ann. Physik. 66, 157.

Byk, A. 1922. Ann. Physik. 69, 161.

Carslaw, H. S. and Jaeger, J. C. 1959. Conduction of heat in solids, 2nd ed. Oxford University, Iondon. 345 .

Challis, L. J. and Sherlock, R. A. 1969. Proceedings of 11th international conference on low temperature physics. Vol. I, 571 .

Cheeke, J. D. N., Herbal, B., Richard, J., and Turkington, R. R. 1973. Phys. Lett. $\underline{46 \mathrm{~A}}, 81$.

Cole, R. 1956. NACA Tech. Note 3885.

Cole, R. 1974. Adv. Heat Transfer. 10, 85.

De Boer, J. and Blaisse, B. S. 1948a. Physica. 14, 149.

De Boer, J. and Lunbeck, R. J. 1948b. Physica. 14, 510.

De Boer, J. and Lunbeck, R. J. 1948c. Physica. 14, 520.

Dickson, D. P. E., Caroline, D., and Mendoza, E. 1970. Phys. Lett. 33A, 139.

Enger, T. and Hartman, D. E. 1972. Third international conference and exhibition on L $\overline{N G}$, Washington, D. C.

Enger, T., Hartman, D. E., and Seymour, E. V. 1973. Adv. Cryog. Eng. 18, 32 . 
Ermakov, G. V. and Skripov, V. P. 1970. High Temp. (USA). 8, 916 .

Fauske, H. K. 1972. Reactor Technol. 15, 278.

Ferrel, R. A. 1951. Phys. Rev. 108, 167.

Fisher, J. 1948. J. Appl. Phys. 19, 1062.

Fisk, S. and Widom, B. 1969. J. Chem. Phys. 50, 3219.

Flory, K., Paoli, R., and Mesler, R. 1969. Chem. Eng. Prog. 65, 50 .

Fowler, W. B. and Dexter, D. I. 1968. Phys. Rev. 176, 337.

Frenkel, J. 1946. Kinetic theory of liguids. Dover Publications, Inc., New York.

Friedman, H. 1962. Advances in chemical physics, Vol. IV. Interscience Publishers, New York.

Frost, W. 1975. Heat transfer at low temperature. Plenum Press, New York.

Fukase, T. and Nishina, Y. 1970. Proceedings of 12th international low temperature conference. E. Kanda (ed.), Academic Press.

Furth, R. 1941. Proc. Cambridge Phil. Soc. 37, 252.

Gaertner, R. F. 1965. J. Heat Transfer. 97, 20.

Genco, J. M. and Lemmon, Jr., A. W. 1970. AFS Trans. 317.

Goodling, J. S. and Irey, R. K. 1969. Adv. Cryog. Eng. $14,159$.

Grigor'ev, V. N. and Rudenko, N. S. 1965. Sov. Phys.-JETP. 20,63 .

Grigoriev, V. A., Klimenko, V. V., Pavlov, Yu. M., Ametisov, Ye. V., and Klimenko, A. V. 1977. Cryogenics. 17, 152.

Guggenheim, E. A. 1945. J. Chem. Phys. 13, 657 .

Haas, G. A. 1972. American Institute of Physics handbook, 3rd ed. , McGraw-Hill. 9-172.

Han, C. Y. and Griffith, P. 1965. Int. J. Heat Mass Transfer. 8,887 . 
Hernandez, J. P. 1973. Phys. Rev. A7, 1755.

Hernandez, J. P. and Silver, M. 1970. Phys. Rev. A2, 1949.

Hord, J., Jacobs, R. B., Robinson, C. C., and Sparks, L. L. 1964. Trans. ASME. A86, 485.

Hsu, Y. Y. and Graham, R. W. 1961. NASA Technical Note D-594.

Iwasa, Y. and Apgar, B. A. 1978. Cryogenics. 18, 267.

Jackson, J. 1969. Cryogenics. 9, 103.

Jergel, M. and Stevenson, R. 1974. Cryogenics. 14, 431.

Johnson, H. A. 1970. Proceedings of the 4th international heat transfer conference, Paris. Abstract B3.1.

Jortner, J., Kestner, N. R., Rice, S. A., and Cohen, M. H. 1965. J. Chem. Phys. 43, 2614.

Kagan, Yu. 1960. Russ. J. Phys. Chem. 34, 42.

Katz, D. L. 1972. Chem. Eng. Prog. 58, 68.

Katz, D. I. and Slipcevich, C. M. 1971. Hydrocarb. Process. 240 .

Kavetskaya, I. V., Sibel'din, N. N., Stopachinskii, V. B., and Tsvektov, V. A. 1979. Sov. Phys. Dokl. 24, 37.

King, P. J. and Wyatt, A. F. G. 1971. Proc. Roy. Soc. A322, 355 .

Kirichenko Yu. A., Tsybul'skii, V. V., Dolgoi, M. I., Rusanov, K. V., and Konovalov, I. M. 1975. J. Eng. Phys. 28, 409 .

Klemens, P. G. 1956. Handbuch der Physik. Vol. 14, 198.

Kopylov, V. N. and Mezhov-Deglin, L. P. 1971. JETP Lett. 14,21 .

Korenblit, I. Ya., Kuznetsov, M. E., Muzhdaba, V. M., and Shalyt, S. S. 1970. Sov. Phys. - JETP. 30, 1009.

Kramers, H. A. 1940. Physica. I, 284.

Kuper, C. G. 1961 . Phys. Rev. 122, 1007. 
Kutateladze, S. S. 1963. Fundamentals of heat transfer. Academic Press, New York.

Kuznetsov, M. E., Oskotskii, V. S., Polshin, V. I., and Shalyt, S. S. 1970. Sov. Phys. - JETP. 30, 607.

Kyotami, Y. 1975. Cryogenics. 15, 372.

Landauer, R. and Swanson, J. A. 1961. Phys. Rev. 121, 1668. Lennard-Jones, J. 1924 . Proc. Roy. Soc. A106, 463. Lifshitz, I. M. 1975. Sov. J. Low. Temp. Phys. 1, 553. Lifshitz, I. M. and Kagan, Y. 1972. Sov. Phys. - JETP. 35,206 .

Long, G. 1957. Metal. Prog. 71, 107.

Lurie, H. and Johnson, H. A. 1962. J. Heat Transfer. 84, 217 .

Lyon, D. N. 1965. Int. Adv. Cryog. Eng. 10, 371.

Marston, P. L. 1976. J. Low Temp. Phys. 25, 383.

Marston, P. L. and Apfel, R. E. 1977. Phys. Lett. 60A, 225.

McCarty, R. D. 1973. J. Phys. Chem. Ref. Data. 2, 923.

Moore, G. R. 1959. AIChE J. $\underline{5}, 458$.

Morimoto, T., Chiba, M., and Takeda, S. 1977. Phys. Lett. $62 A, 107$.

Nakanishi, E. and Reid, R. C. 1971. Chem. Eng. Progr. 67, 36 .

Nelson, W. and Kennedy, E. H. 1956. Paper Trade Journal. 140,50 .

Oker, E. and Merte, Jr., J. 1978. 6th Int. heat transfer conference. Part I. Toronto.

Onn, D. G. and Silver, M. 1971. Phys. Rev. A3, 1773.

Onn, D. G., Smejtek, P., and Silver, M. 1974. J. Appl. Phys. 45, 119 .

Onnes, H. K. and Perrier, A. 1914. Comm. Phys. Lab Leiden. $1390,37$.

Oshima, K. 1974. Adv. Cryog. Eng. 19, 127. 
Parmar, D. S. and Jalaluddin, A. K. 1973. Phys. Lett. 42A, 497.

Parmar, D. S. and Jalaluddin, A. K. 1975. J. Phys. D. 8. 971 .

Pavlov, P. A. and Skripov, V. P. 1965. High Temp. USA. 3. 97 .

Pavlov, P. A. and skripov, V. P. 1967. J. Eng. Phys. $4,267$.

Pavlov, P. A. and Skripov, V. P. 1970. High Temp. USA. 8, 540 .

Porteus, W. and Blander, M. 1975. AIChE J. 21, 560.

Purdy, V., Linnet, C., and Frederking, T. H. K. 1970. Adv. Cryog. Eng. 16, 359.

Rhodes, R. G. and Mulha11, B. E. 1975. Cryogenics. 15, 403.

Roemer, R. B. 1969. Int. J. Heat Mass Transfer. 12, 953.

Rogers, J. D. and Brickweddle, F. G. 1966. Physica 32 , 1001 .

Sakurai, A. and Shiotsu, M. 1977. J. Heat Transfer. 99, 547.

Schmidt, C. 1978. Appl. Phys. Lett. 32, 827.

Schrock, B. E. and Perrais, J. P. 1966. Proc. 1966 heat transfer and fluid mech. inst. M. saeed and J. A. Miller (eds.) Stanford University Press. 122.

Shen, I. and Heberlein, D. C. 1972. Temperature. H. H. Plumb (ed.) Vol. 4, Pt. 2. ISA, Pittsburgh. 791.

Sinitsyn, E. N. and Skripov, V. P. 1968. Russ. J. Phys. Chem. $\underline{42}, 440$.

Skripov, V. P. 1966. Russ. J. Phys. Chem. 40, 1111.

Skripov, V. P. 1974. Metastable liguids. John Wiley and Sons.

Skripov, V. P. and Ermakov, G. V. 1964. Russ. J. Phys. Chem. 38, 208 .

Skripov, V. P. and Sinitsyn, E. N. 1964. Sov. Phys. Usp. I. 887 . 
Skripov, V. P. and Sinitsyn, E. N. 1967. Ukr. Fiz. Zh. $12,99$.

Skripov, V. P. and Sinitsyn, E. N. 1968. Russ. J. Phys. Chem. $42,167$.

Skripov, V. P. and Pavlov, P. A. 1970. High Temp. USA. $\underline{8}, 782$.

Skripov, V. P., Baidakov, V. G., and Kaverin, A. M. 1979. Physica. 95A, 169.

Smejtek, P., Silver, M., and Dy, K. S. 1973 . J. Chem. Phys. 59, 1374 .

Snyder, N. S. 1969. NBS Technical Note 385.

Sommer, W. T. 1964. Phys. Rev. Lett. 12, 271.

Stepanov, V. G., Volyak, I. D., and Tarlakov, Yu. V. 1977. J. Eng. Phys. $32,458$.

Steward, W. G. 1975. AFOSR Interim Report 275.05-75-3.

Steward, W. G. 1978. Int. J. Heat Mass Transfer. 21, 863.

Swanson, J. I. and Bowman, H. F. 1974. Proceedings of 5 th international heat transfer conference. Tokyo. Vol. IV, 60 .

Tobey, G. E., Graeme, J. G., and Huelsman, I. P. 1971. Operational amplifiers - design and applications. McGraw-Hill, New York.

Tokarev, V. M. 1976. Heat Transfer - Sov. Res. $\underline{8}, 17$.

Tolubinskiy, V. I., Ostrovskiy, Yu. N., and Pisarev, V. Ye. 1975. Heat Transfer - Sov. Res. $\underline{7}, 31$.

Tolubinskiy, V. I., Ostrovskiy, Yu. N., and Pisarev, V. Ye. 1976a. Heat Transfer - Sov. Res. 8, 17.

Tolubinskiy, V. I., Ostrovskiy, Yu. N., and Pisarev, V. Ye. 1976b. Heat Transfer - Sov. Res. 8, 29.

Tolubinskiy, V. I., Ostrovskiy, Yu. N., and Pisarev, V. Ye. 1977a. Heat Transfer - Sov. Res. 9, 73.

Tolubinskiy, V. I., Ostrovskiy, Yu. N., and Pisarev, V. Ye. 1977b. Heat Transfer - Sov. Res. 9, 83. 
Tolubinskiy, V. I., Ostrovskiy, Yu. N., and Pisarev, V. Ye. 1978a. Heat Transfer - Sov. Res. 10, 74.

Tolubinskiy, V. I., Ostrovskiy, Yu. N., and Pisarev, V. Ye. 1978b. Heat Transfer - Sov. Res. 11, 1 .

Tolubinskiy, V. I., Ostrovskiy, Yu. N., and Pisarev, V. Ye. 1978c. Heat Transfer - Sov. Res. 11, 18.

Tsukamoto, O. and Kobayashi, S. 1975. J. Appl. Phys. 46, 1359.

Uhlman, D. R. and Chalmers, B. 1965. Ind. Eng. Chem. 57, 19.

Van der Waals, J. D. 1894. Z. Physik. Chem. 13, 657.

Van Dijk, H., Durieux, M., Clement, J. R., and Logan, J. K. 1960. J. Res. Nat. Bur. Std. 64A, 4 .

Verkin, B. I., Kirichenko, Yu. A., Dolgoy, M. L., Levchenko, N. M., and Tsybulsky, V. V. 1976. Proceedings of 6th international cryogenic engineering conference, Grenoble, France. 286 .

Volmer, M. 1939. Kinetic der Phasenbildung. Steinkoff, Dresden-Leipzig; Engl. transl. Kinetics of phase transformation. ATI No. 81935 (F-TS-7068-RE). Clearinghouse Fed. Sci. Tech. Inform. Springfield.

Wakeshima, H. and Takata, K. 1958. J. Phys. Soc. Jap. 13,1398 .

Widom, B. 1965 . J. Chem. Phys. $43,3892$.

Witte, L. C., Cox, J. E., and Bouvier, J. E. 1970. J. Met. 39.

Woolf, M. A. and Rayfield, G. W. 1965. Phys. Rev. Lett. 15,235 .

Yonemitsu, K. 1979. Butswri. $34,771$.

Young, T. 1960. Phys. Rev. 117, 1244.

Zel'dovich, Ya. B. 1943. Acta Physiochim. URSS. 18, 1.

Zitter, R. N. 1965a. Phys. Rev. Lett. 14, 14.

Zitter, R. N. 1965b. Phys. Rev. Lett. 139A, 2021. 


\section{APPENDIX A}

At the present time surface tension data for only a limited number of hydrogen isotopes are available. These data cover only a narrow temperature range, usually far away from the critical temperature. No surface tension data for $T_{2}$ is available. In the absence of experimental data one may resort to the use of the law of corresponding states for an estimate of surface tension values with reasonable accuracy. The empirical relation

$$
\gamma(T)=\gamma_{0}\left(1-\frac{T_{C}}{T_{C}}{ }^{\mu}\right.
$$

suggested by van der Waals (1894) appears to describe the simple fluids remarkably well. Expression (AI) has been subsequently shown by Guggenheim (1945) to arise as a natural product of the law of corresponding states. The measured surface tension data over a wide temperature range for $\mathrm{Ar}, \mathrm{N}_{2}$, and Xe fit (AI) to within 1.6 per cent for $\mu$ in the range $1.25-1.29$. The value of $\mu=1.5$ originally predicted by van der waals (1894) is thus too high, while the value suggested by Guggenheim (1945) (1.222) and widom (1965), and Fisk and Widom (1969) (1.20) are much too low for the classical liquids. For quantum liquids such as Ne, ${ }^{4} \mathrm{He}, \mathrm{H}_{2}$ and its isotopes the value of $\mu$ is significantly 
different from that predicted by theories and from that corresponding to the classical liquids.

According to the classical principle of corresponding states we should expect the reduced surface tension $\gamma^{*}=\gamma \sigma^{2} / \varepsilon$ to be a universal function of the reduced temperature $\mathrm{T}^{*}=\mathrm{kT} / \varepsilon$, for those substances which are described well by the Lennard-Jones 6-12 potential. Hirschfelder et al. (1954) showed that the reduced surface tension $\gamma^{*}$ depends parametrically on the quantum mechanical de Boer parameter $\Lambda$. The phenomenological relation (A1) was modified by them as

$$
\gamma^{*}=\alpha(\Lambda)\left(1-\frac{T^{*}}{T_{C}^{*}}\right)^{\mu}
$$

where the coefficient $\alpha$ introduces the $\Lambda$-dependence. Unfortunately, they assumed a single value of $\mu=11 / 9$ for all liquids, thus predicting the same temperature dependence for both classical and quantum fluids which is not supported by the experimental data. Moreover, they did not take into account any modification of the quantum parameter $\Lambda$ for the heteronuclear molecules such as HD and DT (Friedman 1962).

We suggest that $\mu$ should also explicitly depend upon 1. Consequently, the relation between the reduced surface tension $\gamma^{*}$ and reduced temperature $T^{*}$ should be modified as 


$$
\gamma^{*}=\alpha(\Lambda)\left(1-\frac{T^{*}}{T_{C}^{*}}\right)^{\mu(\Lambda)}
$$

Skripov and Sinitsyn (1968) have shown that the surface tension can be determined quite accurately from the measured homogeneous nucleation temperature of a liquid. They have also verified that the small interface curvature ( $50 \AA)$ of the critical nuclei involved with the homogeneous nucleation in a superheated liquid, does not seem to influence the estimated surface tension value as compared to the directly measured surface tension. We have used this method to independently derive the surface tension for several hydrcyen isotopes and test the validity of expression (A3). The primary advantage of this method is that the estimated surface tension corresponds to the surface tension of temperatures close to the critical $\mathrm{T}_{\mathrm{C}}$ of the liquids. This is because the homogeneous nucleation temperatures are usually in the range between $.85 \mathrm{~T}_{\mathrm{C}}$ and $T_{C} \cdot$ Consequently, the correct temperature dependence of surface tension in the above temperature range near the critical point can be determined reliably. The nucleation rate $\mathrm{J}$ nuclei/ $\mathrm{cm}^{3} / \mathrm{sec}$ predicted from the homogeneous nucleation theory (see Chapter III) for all the hydrogen isotopes can be expressed with sufficient accuracy in the following form 


$$
\ln J=76-\frac{16 \pi \gamma^{3}}{3 k T\left(P_{V}-P_{L}\right)^{2}}
$$

for $J=1$, the expression (A4) reduces to a simpler form

$$
\gamma(T)=\left[4.53 k\left(P_{V}-P_{L}\right)^{2} T\right]^{\frac{1}{3}}
$$

We have obtained the homogeneous nucleation temperature data for the hydrogen isotopes from a quantum law of corresponding states as applied to the case of homogeneous nucleation. As previously mentioned in Chapter III, the advantage of this quantum law of corresponding states is that the homogeneous nucleation temperature of any hydrogen isotope can be predicted at any given reduced pressure $\mathrm{P}^{*}$ knowing only the de Boer parameter for the isotope. Since $\gamma \propto T^{\frac{1}{3}}$, the error involved in predicting $T$ does not appreciably affect the estimated value of surface tension. This can be seen from Fig. 38 which shows the estimated surface tension data from homogeneous nucleation temperature (A5) and the experimental data of Grigor'ev and Rudenko (1965) for $D_{2}$. The agreement is satisfactory. We also find that the interpolated value of $\mu$ obtained from the $\Lambda$ dependence of $\mu$ as shown in Fig. 39 is consistent with the value obtained from (A5) thus justifying our previous suggestion (A3). This consistency has been checked with other hydrogen 


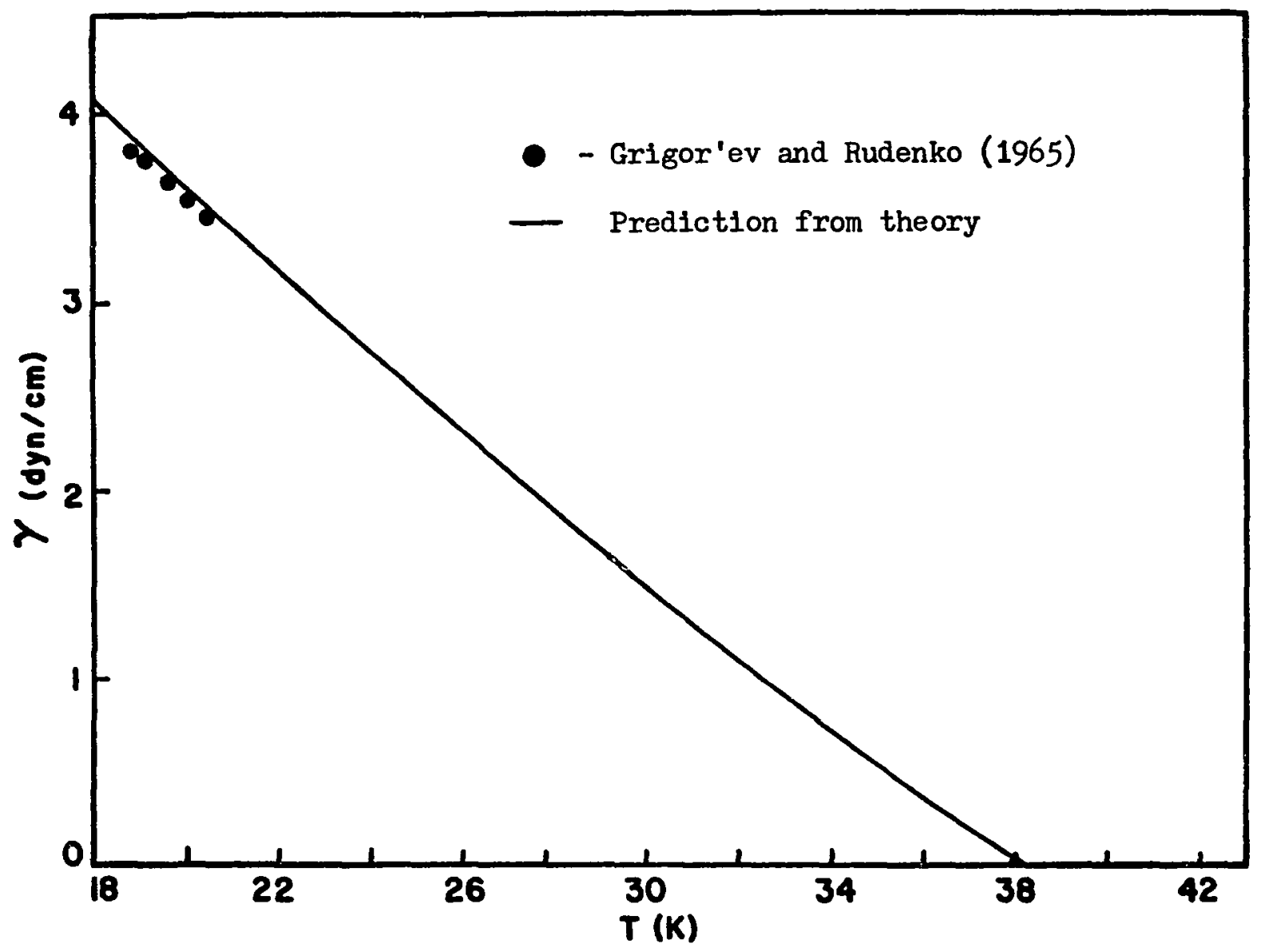

Figure 38. Surface tension of deuterium as a function of temperature. The predicted surface tension is compared with experimental data of Grigo'ev and Rudenko (1965). 


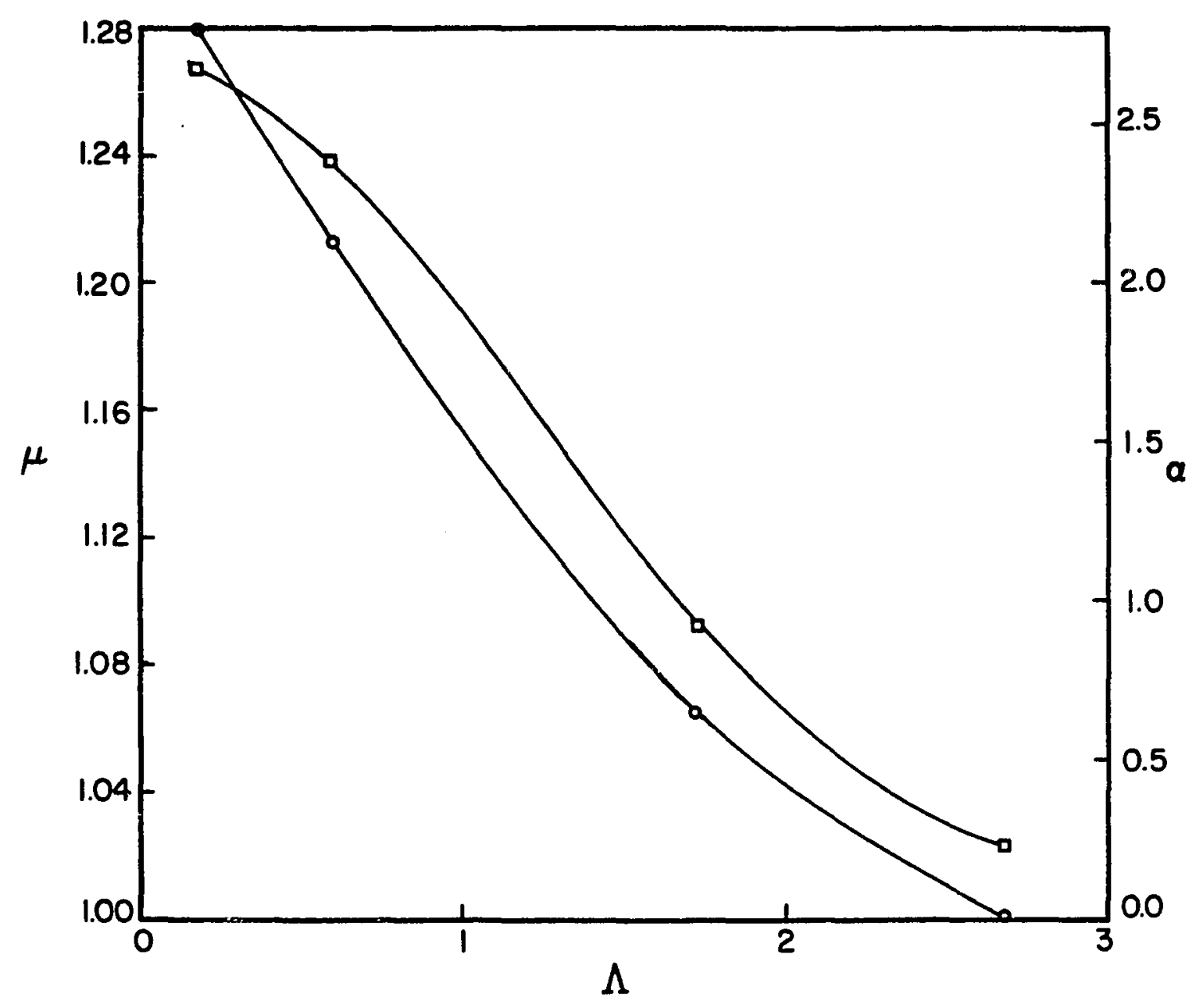

Figure 39. A plot of $\mu$ and $\alpha$ as a function of the de Boer parameter. The dependence of $\mu(O)$ and $\alpha(\square)$ on the de

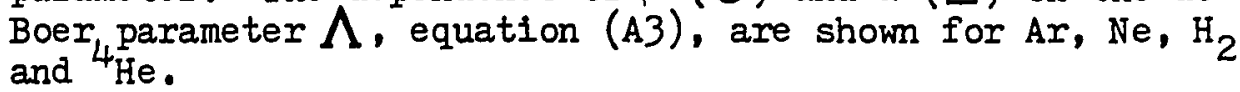


isotopes as well. For tritium we find the surface tension can be expressed as

$$
\gamma(T)=9.49\left(1-\frac{T}{T_{C}}\right)^{1.146} \mathrm{dyn} / \mathrm{cm} .
$$




\section{APPENDIX B}

The thermodynamic condition for the spinodal curve $(\partial P / \partial V)_{T}=0$ defines the limit of stability of a homogeneous one-component system with respect to relatively small mechanical and thermal perturbations. Thus, the spinodal demarcates the metastable and the absolutely unstable states of a phase. The spinodal is not experimentally realizable in either superheated or tensilely stressed one-component liquids because of the more restrictive kinetic limit of stability associated with homogeneous nucleation of the vapor phase. Recently, however, Parmar and Jalaluddin (1973, 1975) reported an experimental method for the determination of the spinodal of liquids by extrapolating the maximum superheat values obtained from electric-fieldinduced nucleation temperatures. They found that the temperature at which nucleation occurred depended on the applied electric field and decreased with increasing field. Marston and Apfel (1977) have questioned the validity of such an experiment in inferring the spinodal. They argued on a theoretical basis that the electric field should inhibit homogeneous nucleation, contrary to what had been experimentally observed (Parmar and Jalaluddin 1973, 1975). Consequently, they believe that the observed field-induced nucleation was presumably affected either by properties 
of the electrode-liquid interface or by a lower dielectric strength of the liquids.

One inherent difficulty in the above experiment appears to be the presence of an electrode in the superheated liquid. In the study of electric-field-induced nucleation such a situation cannot be avoided. We suggest an alternative approach to this problem. We feel that it may be appropriate to study the effect of an external magnetic field on the homogeneous nucleation temperature of a paramagnetic substance such as liquid oxygen. The obvious advantage of this approach is that no electrodes inside the liquid would be necessary.

According to the homogeneous nucleation theory (see Chapter III) the nucleation rate $J$ depends exponentially on the work of formation of a critical nucleus,

$$
J=\beta \exp \left(-\bar{w}_{c r} / k T\right)
$$

In the absence of a magnetic field, the work of formation of a critical nucleus is given by (see Chapter III)

$$
w_{c r}=\frac{16 \pi \gamma^{3}}{3 k T\left(P_{e}-P_{L}\right)^{2} \delta^{2}}
$$

where $P_{e}$ is the equilibrium vapor pressure, $P_{I}$ the ambient pressure, and $\gamma$ the surface tension. The Boynting correction $\delta$ relates $\mathrm{P}_{\mathrm{v}}$ to the equilibrium vapor pressure 
$P_{e}: \delta=\left(P_{v}-P_{L}\right) /\left(P_{e}-P_{L}\right)$. The $P, T$ dependence of the exponential dominate that of the prefactor $\beta$ in determining the nucleation rate. Maximum observable superheats, obtained by the transient superheating technique previously mentioned in Chapter IV, for liquid nitrogen and oxygen are described well by (B1).

An applied magnetic field $B$ introduces a change in J primarily through the exponential. The magnetostatic energy of a spherical bubble with radius $r$ in a uniform field is given by

$$
w_{B}(v)=\frac{2 \pi v^{3}\left(1+x_{\ell}\right)\left(x_{\ell}-x_{v}\right) B^{2}}{\mu_{0}\left(3+x_{v}+2 x_{\ell}\right)},
$$

where $x_{\ell}$ and $x_{v}$ are the susceptibilities of the liquid and the vapor within the bubble, respectively, and $\mu_{0}$ is the magnetic permeability of free space. In an applied field the work of formation of a critical bubble becomes

$$
w_{c r}^{\prime}=w_{c r}+w_{B}\left(r_{c r}\right)
$$

which may be expressed as

$$
w_{C r}^{\prime}=\frac{16 \pi \gamma^{3}}{3 k T\left(P_{e}^{\prime}-P_{I}-P_{B}\right)^{2} \delta^{2}}
$$


where

$$
P_{B}=\frac{3\left(1+x_{\ell}\right)\left(x_{\ell}-x_{v}\right) B^{2}}{\mu_{0}\left(3+x_{v}+2 x_{\ell}\right)}
$$

and

$$
x_{\ell} \gg x_{v} \text { (Onnes and Perrier 1914) }
$$

Nucleation parameters modified by the magnetic field are indicated with primes. Since $w_{B}\left(r_{C r}\right)$ is a positive quantity, i.e. $W_{C r}^{\prime}>W_{C r}$, the nucleation rate in the magnetic field

$$
J^{\prime} \simeq \beta \exp \left(-W_{C r}^{\prime} / k T\right)
$$

must be lower than $\mathrm{J}$ for $\mathrm{a}$ fixed $\mathrm{P}$ and $\mathrm{T}$. For any given nucleation rate $J=J^{\prime}$, the homogeneous nucleation temperature is increased above their zero field values. For liquid oxygen this increase in the nucleation temperature is estimated to be $\geqslant 1 \mathrm{~K}$ at atmospheric pressure and at a magnetic field of 2 Tesla. Such a change in temperature should be easily observable in the capillary heating method employed by skripov et al. (1979) for determining the maximum attainable superheat in cryogenic liquids which has a temperature resolution of the order of $0.1 \mathrm{~K}$. 


\section{APPENDIX C}

This superheat temperature $\Delta T$ as a function of time $t$ of a wire of radius $r$ homogeneously heated in a medium at constant power is given by Carslaw and Jaeger (1959) as

$$
\Delta T(t)=\frac{2 g \alpha^{2}}{\pi^{3} \lambda} \int_{0}^{\infty} \frac{1-\exp \left(-a t u^{2} / r^{2}\right)}{u^{3} \Delta(u, \alpha)} d u
$$

where,

$$
\begin{aligned}
q= & \text { power dissipated per unit length of the wire } \\
\lambda= & \text { thermal conductivity of the medium } \\
a= & \text { thermal diffusivity of the medium } \\
\alpha= & 2 \rho c / \rho_{w} c_{w^{\prime}} \rho \text { and } c \text { the mass density and the } \\
& \text { specific heat of the medium, and } \rho_{w^{\prime}} c_{w} \text { the } \\
& \text { corresponding quantities for the wire. }
\end{aligned}
$$

The function $\Delta(u, \alpha)$ is defined in terms of Bessel function as

$$
\Delta(u, \alpha)=\left[u J_{0}(u)-\alpha J_{1}(u)\right]^{2}+\left[u Y_{0}(u)-\alpha Y_{1}(u)\right]^{2}
$$

Approximations of (Cl) are available which are valid either for large times at $/ x^{2} \gg 1$ or for short times at $/ r^{2} \ll 1$. Unfortunately, the time scales of the transient superheating 
experiment fall in between the two limits for which no simple approximate solution is available.

For convenience in comparing the predicted temperature response of a thin wire with that observed in the transient superheating experiments, the expression (C1) has been evaluated numerically on the computer. The values were curve fitted using the least squares method in the following form

$\frac{\Delta T(t) \lambda}{q}=4.803 \times 10^{6}\left(t-24.773 t^{1.5}+44.09 t^{1.6}-20.33 t^{1.7}\right)$

The expression (C3) provides an excellent approximation to (C1) within .2 per cent for time periods up to $300 \mathrm{~ms}$. Above $300 \mathrm{~ms}$ the large time scale approximation is valid. The expression (C3) is derived for a platinum wire with $\alpha=1.79$, radius $r=.0051 \mathrm{~cm}$ and thermal diffusivity ' $a$ ' commensurate with the values for liquid nitrogen. 